\title{
PROTECTIVE EFFECTS OF MILK PHOSPHOLIPIDS AGAINST UV PHOTODAMAGE IN HUMAN SKIN EQUIVALENTS
}

\author{
A Thesis \\ presented to \\ the Faculty of California Polytechnic State University, \\ San Luis Obispo
}

\author{
In Partial Fulfillment \\ of the Requirements for the Degree \\ Master of Science in Biomedical Engineering
}

by

Zyra Mae L. Achay

September 2011 
(C) 2011

Zyra Mae L. Achay

ALL RIGHTS RESERVED 


\section{COMMITTEE MEMBERSHIP}

TITLE:

AUTHOR:

DATE SUBMITTED:

COMMITTEE CHAIR:

COMMITTEE MEMBER:

COMMITTEE MEMBER:
Protective Effects OF Milk Phospholipids against UV Photodamage in Human Skin Equivalents

Zyra Mae L. Achay

September 2011

Lily Hsu Laiho, Ph.D.

Kristen O’Halloran Cardinal, Ph.D.

Elena Keeling, Ph.D. 


\begin{abstract}
Protective Effects of Milk Phospholipids against UV Photodamage in Human Skin Equivalents Zyra Mae L. Achay
\end{abstract}

The ultraviolet (UV) spectrum has been known to cause damage to skin in varying degrees. UVB radiation (290-320 nm) in particular, has been proven to be highly mutagenic and carcinogenic in many animal experiments compared to either UVA or UVC. The alarming rate of increase in skin cancer incidence has prompted many investigators to pursue other alternatives to sunscreens including changes in lifestyle habits and dietary consumption in order to boost our efforts in tackling this widespread disease. Previous studies employing confocal reflectance, MTT assay and histology suggest that milk phospholipids may possess protective properties against UVB-mediated damage but the molecular mechanism for this effect remains unclear. This study aims to evaluate changes in cell morphology, apoptosis and p21 expression in tissue engineered epidermis to increase our understanding of the mechanisms behind the potential protective effects of milk phospholipids against UV-induced photodamage. Human skin tissue equivalents were incubated in either $1 \%$ milk phospholipid solution or maintenance media then exposed to $120 \mathrm{~mJ} / \mathrm{cm}^{2}$ dose of $300 \mathrm{~nm}$ UVB after 24 hours. The upregulation of $\mathrm{p} 21$ protein in response to DNA damage was measured with Western blot and immunofluorescence microscopy and markers for apoptosis and hyperplasia were examined 24 hours after irradiation. Results revealed that p21 levels and the amount of apoptotic markers such as fragmented DNA and nuclear condensation were significantly reduced in UV-exposed tissues pre-incubated with milk phospholipids compared to levels seen in both the positive control and UV-exposed skin tissue not incubated with milk phospholipids. This decrease in p21 expression may imply a reduction in DNA damage 24 hours after UV exposure or a decrease in acquired photodamage at the outset. Milk phospholipid incubation however, induced an increase in epidermal thickening with or without UV exposure, which may imply induction of a protective mechanism to enhance the barrier properties of skin.

Keywords: phospholipids, skin cancer, p21, skin equivalent, UV radiation 


\section{ACKNOWLEDGMENTS}

I am deeply grateful to my thesis advisor and mentor, Dr. Lily Laiho, for being an inspiration, for your vast knowledge, generous guidance, support and encouragement, for the unwavering patience, for the funding, my first conference ever and for making it possible for me to become part of an awesome project.

I thank my committee members, Dr. Elena Keeling - for always being willing to share your expertise in Biology and for thorough advice on every aspect of my thesis, Dr. Kristen O'Halloran Cardinal - for all your input, for everything I learned in my classes and for training me especially in cell culture and tissue engineering. Thank you for taking the time to read my thesis draft and providing insightful comments on my manuscript and presentation.

I would also like to acknowledge my fellow labmates and project collaborators Carl Dargitz, Michael Bingham and Ashley Russell for practically doing all the experiments with me, for sharing your ideas, all the invaluable help, hard work, encouragement, support, company and friendship.

To the Dairy Science team, especially Dr. Rafael Jimenez-Flores, Erin Stefanutti and Andrea Laubscher for providing the milk phospholipids, all the technical advice and training, and for allowing me to work and use equipments and reagents in the protein, chemistry and microbiology lab for the duration of the project.

And lastly, to my family and friends both here and back home - for the unending love, dedication, understanding, moral, financial and spiritual support. And to God who made everything possible from start to finish. 


\section{TABLE OF CONTENTS}

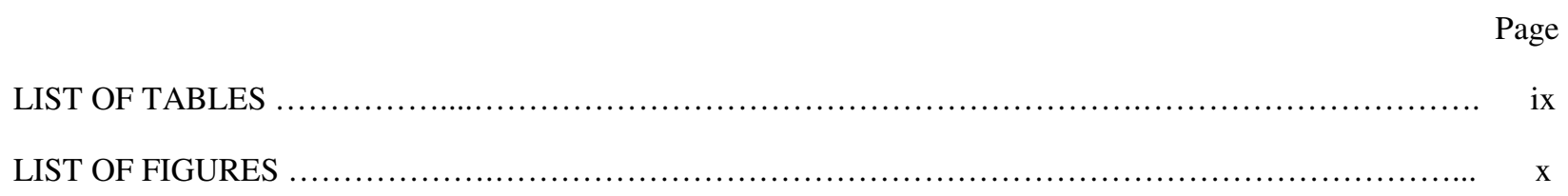

\section{CHAPTER}

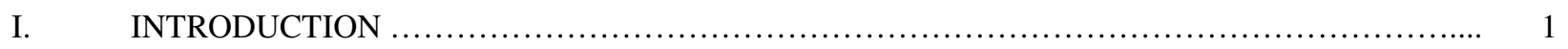

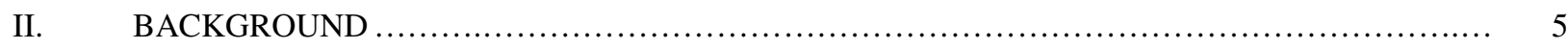

Human Skin Structure and Function ................................................ 5

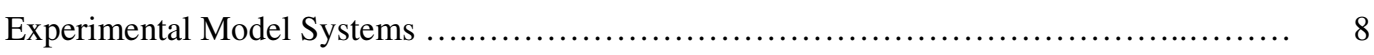

Murine Models ................................................................ 9

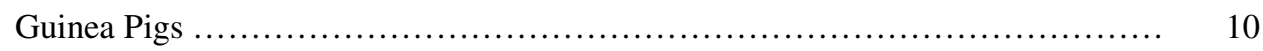

Cultured Cells and Cell Lines .............................................. 11

Ex Vivo Skin Models ....................................................... 13

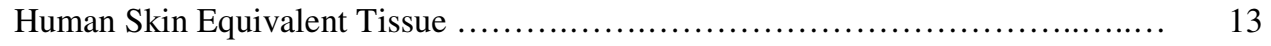

UV Radiation and its Immediate Effects .......................................... 14

Biological Effects of Solar UV .................................................... 17

Effects of UV on Tissue Morphology ....................................... 18

Erythema.......................................................... 18

Pigmentation..................................................... 20

Autofluorescence.................................................... 21

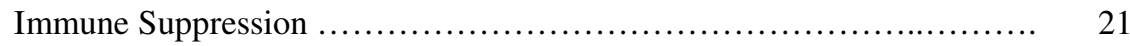

Photoaging ...................................................... 22

Hyperplasia and Epidermal Thickening ............................. 23

Cellular and Molecular Effects ............................................... 24

Reactive Oxygen Species...................................... 27

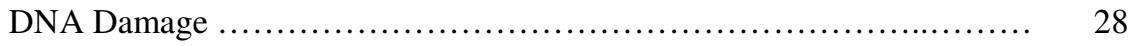

Skin Carcinogenesis....................................................... 29

Tumor Suppressor and Oncogenes.................................. 31 
Types of Skin Cancer............................................ 31

UV Protective and Repair Mechanism............................................ 34

Protective Barrier.............................................................. 34

Antioxidants......................................................... 35

p55-mediated Cell Cycle Arrest......................................... 37

DNA Repair............................................................. 39

Apoptosis................................................................... 41

Phospholipids Structure and Function............................................. 44

Phospholipid Components in the Epidermis.......................................... 46

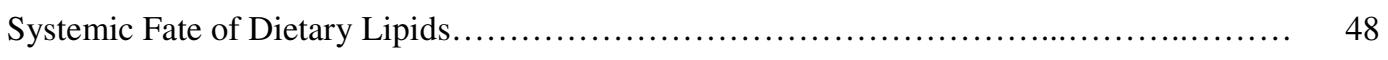

Potential Anticarcinogenic Lipids................................................. 49

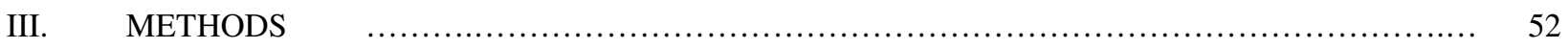

Milk Phospholipid Mixture........................................................ 52

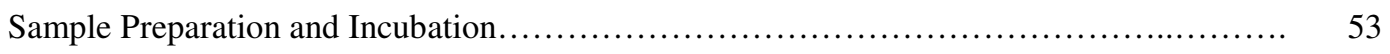

UV Irradiation .............................................................. 55

Hoechst and Immunofluorescence Staining ...................................... 56

Tissue Fixation and Chemical Processing .................................. 56

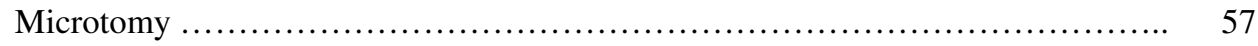

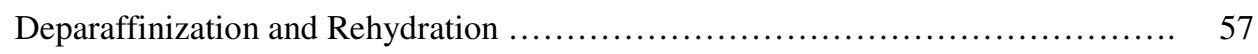

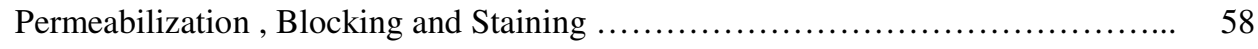

Laser Scanning Confocal Microscopy ...................................... 59

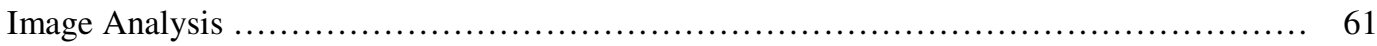

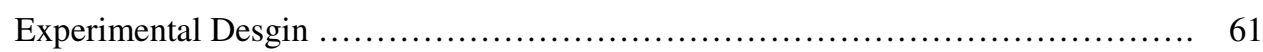

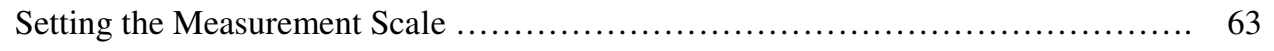

Measurement of Epidermal Thickness ......................................... 64

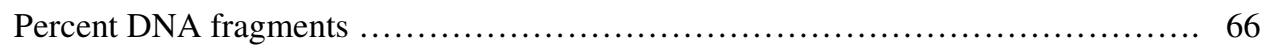

Analysis of p21 Immunofluorescence-Positive Cells ........................... 67

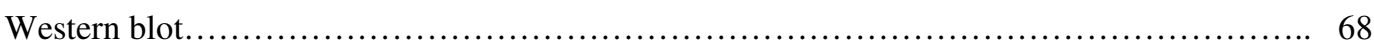

Tissue Lysis and Total Protein Extraction..................................... 68 
Protein Quantification................................................... 70

Sodium Dodecyl Sulfate - Gel Electrophoresis............................ 72

Coomassie Blue Staining................................................. 73

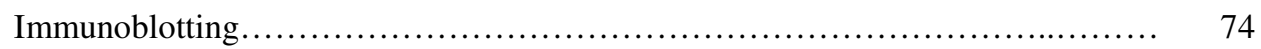

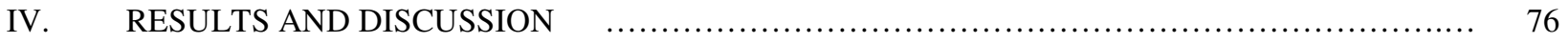

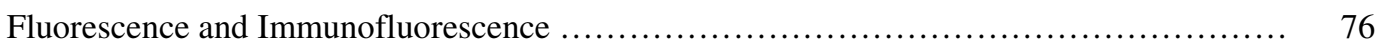

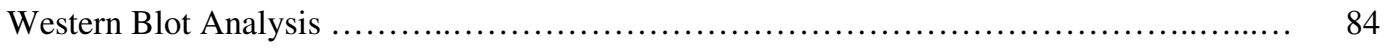

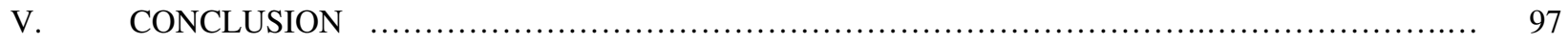

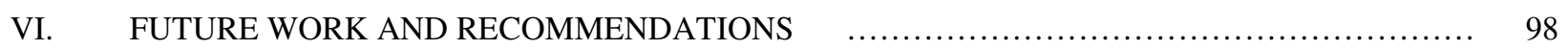

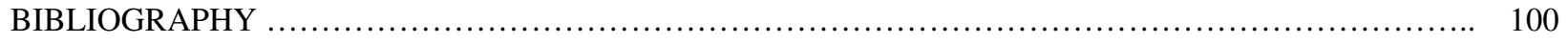

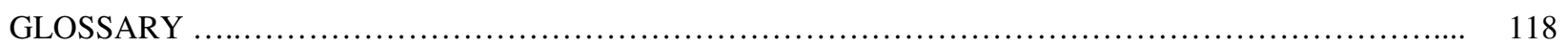

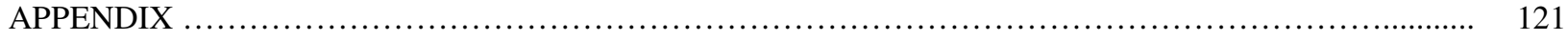




\section{LIST OF TABLES}

Table

Page

1. Phospholipid profile (mol\% of total phospholipids) of milk phospholipid concentrate (PC-700) analyzed with 2D-TLC and ${ }^{31}$ P NMR....

2. Designated volumes of Bradford Assay protein standards from a $1 \mathrm{mg} / \mathrm{ml} \mathrm{BSA}$ standard stock solution. 70

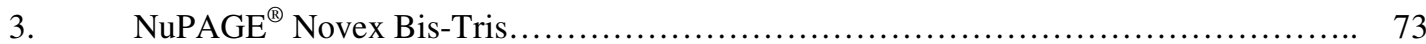

4. Representative Bradford Assay absorbance values of BSA standards and tissue samples after $2 \%$ SDS extraction procedure followed by TCA/acetone precipitation......

5. Relative band intensity analysis of the gel and chemiluminescent blot................ 90 


\section{LIST OF FIGURES}

Figure

Page

1. Non-linear relationship between sun protection factor and sunscreen

concentration

2. Layers of the epidermis

3. Electromagnetic spectrum showing the relationship of wavelengths

from radio to gamma waves.

4. Skin penetration depth of ultraviolet radiation of different wavelengths.

5. CIE action spectrum for the susceptibility of the Caucasian skin to

Erythema.

6. Structure of (a) a cyclobutane pyrimidine dimer and (b) 6-4 photoproduct...... . 26

7. Basal and squamous cell carcinoma................................... 32

8. p53 accumulation and cell cycle proteins................................ 39

9. Chronological steps involved in nucleotide excision repair ..................... 41

10. $\quad$ p53-dependent and independent apoptotic pathways $\ldots \ldots \ldots \ldots \ldots \ldots \ldots \ldots \ldots \ldots \ldots \ldots$

11. Phospholipid membrane bilayer components ........................... 45

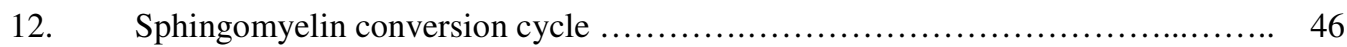

13. EpiDerm ${ }^{\mathrm{TM}}$ reconstructed human epidermis $(\mathrm{RhE})$ tissue model with

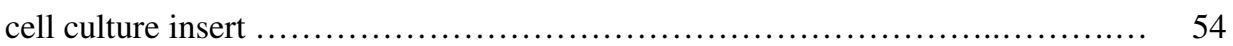

14. Set-up for UV irradiation of tissue engineered skin sample..................... 56

15. Image of Hoechst dye binding to the minor groove $\ldots \ldots \ldots \ldots \ldots \ldots \ldots \ldots \ldots \ldots \ldots$

16. Approximate fluorescence excitation and emission of Hoechst 34580 dye......... 60

17. Minitab General Linear Model dialog box entries for obtaining the standard error for the pilot study

18. Power and sample size values for determining the ideal sample size for \%DNA

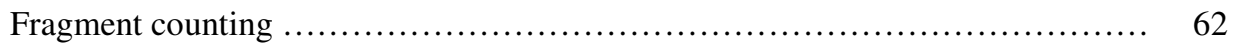

19. Setting the measurement scale using the straight line tool and set scale option .... 63 
20. Dialogue box entries or modifying set scale settings for the image .............. 64

21. Epidermal thickening measurement using the straight line tool of ImageJ ........ 65

22. ImageJ cell counter plug-in for \%DNA fragmentation measurements ............ 66

23. Blue and red channel of a UV sample image................................ 67

24. DAPI and Alexa ${ }^{\circledR}$ Fluor 647 channels merged using the image calculator "AND” function then converted to "brgbcmyw" ................................... 68

25. Power curve plot for sample size estimation and design of experiments with

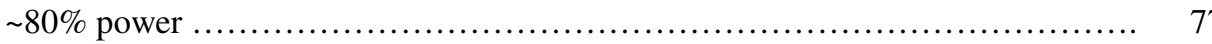

26. Representative images for epidermal thickness comparison ..................... 77

27. Boxplot of epidermal thickness measurements for each treatment group .......... 78

28. Individual effects plot of milk incubation and UV irradiation on epidermal

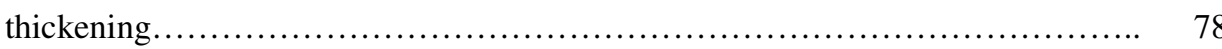

29. Interaction plot showing the lack of depence between the two treatment factors

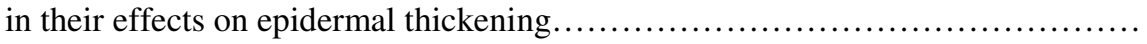

30. Representative images of cells exhibiting nuclear condensation (A) and DNA fragmentation (B) as indicated by the yellow arrowheads.................... 80

31. Boxplot of DNA fragmentation effects of milk phospholipid and UV treatments.. 81

32. Interaction plot showing the dependence of UV radiation's DNA fragmentation effect on prior milk phospholipid incubation................................ 81

33. Effect of phospholipid and UV treatment on the amount of p21 ................ 83

34. Interaction between Phospholipid and UV treatments showing the dependence of UV treatment's p21 upregulation effects on milk phospholipid incubation......

35. Standard curve plot of actual individual Bradford Assay absorbance values using BSA standards of increasing concentration.

36. Western blot optimization of blocking step with positive control and UV samples... 87

37. Western blot analysis of p21 expression in human skin tissue equivalents.......... 88

38. ImageJ gel analysis of chemiluminescent blot $\mathrm{p} 21$ bands....................... 89 


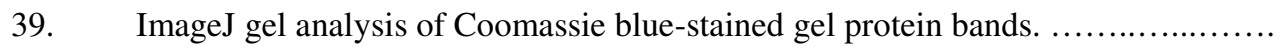

40. Western blot analysis of negative control, positive control and melanoma

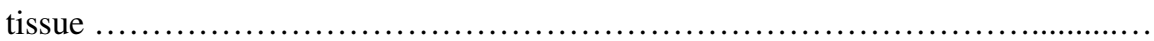




\section{Chapter I. Introduction}

The impact of solar UVB radiation in humans has been profound and has caused approximately $1,000,000$ cases of nonmelanoma skin cancer each year (Miller and Weinstock, 1994). Its wavelength ranges from 280-320 $\mathrm{nm}$, which can cause sunburn and induce photoproducts and pyrimidine dimer formation. These may eventually lead to mutations in the DNA, which when left unrepaired, can trigger cell death or skin carcinogenesis.

Among all the tumors in Caucasians, skin cancer is the most prevalent and its lifetime risk is approximately as high as all other cancers combined (Kraemer, 1997). In 2010 in the U.S. alone, around 68,130 individuals were diagnosed with melanoma with an approximate mortality of 8,700 patients ("Melanoma," 2011). Nearly \$2 billion is spent treating melanoma every year, and though mortality from non-melanoma skin cancers is low, its annual cost due to disfigurement and medical bills is estimated to be over $\$ 500$ million annually. These numbers are even expected to rise because of increased interest in outdoor recreation such as skin tanning, migration to regions with higher sun intensity and ozone depletion (McKenzie et al. 1999).

Alternative methods to protect the skin from photodamage due to solar radiation are still being pursued to advance efforts in combating against skin cancer and photoaging. Sunscreens have long been considered the "gold standard" in conferring protection against photodamage (DeBuys et al. 2000), yet they may provide inadequate protection and are even possibly harmful due to inconsistent and flawed usage, limited spectral protection and the presence of toxic ingredients such as oxybenzone (Pinnell 2003). Indeed, recent efforts of the FDA to push revisions on sunscreen regulations (Food and Drug Administration [FDA], 2011) invite rethinking on the effectiveness of currently existing sun protection methods. Chemicals currently present in many sunscreens have been shown to generate free radicals upon UV exposure (Xu et al. 2001). Recent studies (Wulf et al, 1997) also reveal that SPF is tested at skin applications of $2 \mathrm{mg} / \mathrm{cm}^{2}$, whereas actual sunscreen applications in controlled studies are only 0.5 $\mathrm{mg} / \mathrm{cm}^{2}$ at maximum (Autier et al. 2001). In such amounts and because of the non-linear proportion between SPF and concentration (Figure 1), no sunscreen is known to actually confer beyond an SPF 4 protection. Additionally, sunscreen chemicals are usually tested and measured against erythema or skin reddening, but more importantly, DNA damage manifested through various markers such as thymine dimer formation, 8-hydroxy-2'-deoxyguanosine formation, p53 induction and immunosuppression occurs below minimum levels of UV dose that trigger erythema. Thus, the use of sunscreens in reality has not lived up to the protective merits it is often accorded with and 
inappropriate usage may even contribute to the dramatic increase in skin cancer incidence ("Incidence of skin cancer rising at alarming rate", 2011).

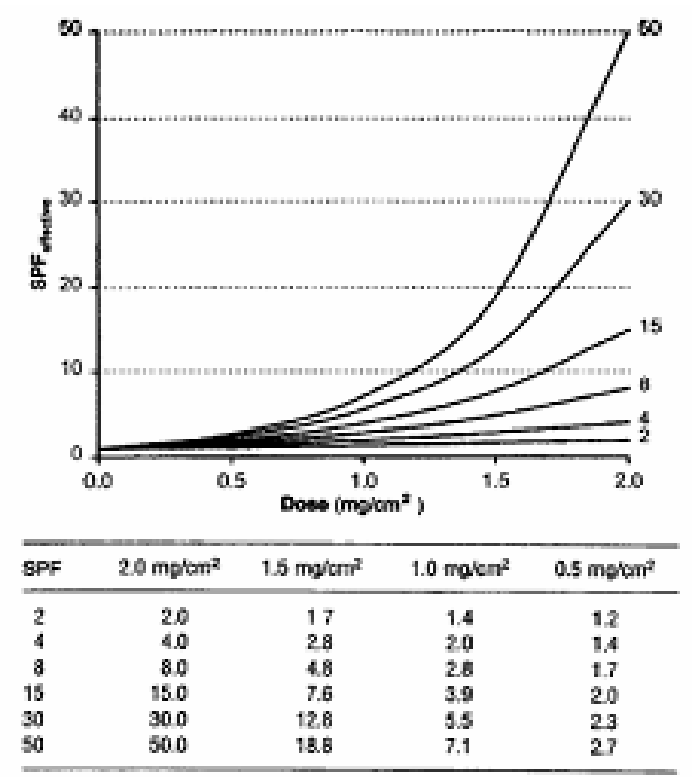

Figure 1. Non-linear relationship between sun protection factor and sunscreen concentration. Source: Wulf et al. (1997).

Changes in lifestyle including dietary habits are strongly believed to be keys in the prevention of at least two-thirds of cancer incidences (Ames et al, 1995; Potter, 1997; Sporn and Suh, 2002). In regards to UVB-induced skin carcinogenesis, this hypothesis has been tested in several mice studies using consumable chemicals and substances such as green tea, black tea (Wang, Huang et al. 1994), soybean symbiosome (Gruber et al. 2004), epigallocatechin galate (Xia et al. 2005), liposomal endonuclease (Nay et al. 2006), silymarin (Katiyar et al. 1997) and silibinin (Dhanalakshmi et al. 2004). Some of the pathways by which these compounds exert their effects include regulating the production of oxidation dimers or formation of other DNA photoproducts due to UV irradiation of skin and increasing repair activity at photodamaged sites.

Phospholipids are only recently being recognized as highly bioactive molecules of increasing importance due mainly to the key roles they play in regulatory pathways and possibly even carcinogenesis (Merrill, Schmelz et al. 1995). Sphingomyelin and other sphingolipids in particular, have been discovered to participate in processes involved in cancer progression, namely, cell growth, differentiation and apoptosis. Yet further studies are needed since little is known so far about the mechanisms of action of these compounds, much less, their systemic fate after oral ingestion and how their consumption affects other organs in the body. Ideal solar defense agents should possess 
photoprotective properties including the reduction of erythema and sunburn formation, DNA changes including thymine dimers and oxidized nucleotides, UV immunosuppression, hypo- and hyperpigmentation and ultimately, skin cancer and photoaging. The team of Dr. Rafael Jiménez-Flores at the Dairy Science Department of California Polytechnic State University has been working on various protocols to extract and purify liposomes from bovine milk phospholipids and have initiated efforts to evaluate potential protective effects of milk phospholipid liposomes against UV photodamage. Preliminary studies involving confocal reflectance, MTT assay and histology have pointed towards milk phospholipids' potential in protecting human skin equivalents against UV photodamage (Russell et al. 2010). However, the mechanisms of milk phospholipid action have yet to be elucidated.

Since most skin cancers develop in sun-exposed regions of the skin, studies on the response mechanisms to photodamage due to UV light exposure in skin models are of prime importance. Use of in vitro skin equivalents is particularly useful due to their high consistency per sample lot, thus reducing confounded experimental variables. It also facilitates extrapolation of results to human cases as opposed to using cultured cells or murine models. At the same time, the necessity of complying with legal, sociopolitical and ethical directives associated with using human skin biopsies and animal models are circumvented.

Increased expression of p53 protein and its downstream effectors including p21 in normal skin are known to occur in response to DNA damage and results in cell cycle arrest or apoptosis (Kuerbitz et al. 1992; Lowe et al. 1993; Clarke et al. 1993). Mutations in the p53 gene, on the other hand, arise with chronic UVB irradiation. The expression of p21 is implicated in the regulation of cell cycle and DNA replication, integrity and repair. Increase in p53/p21 levels is associated with $\mathrm{G}_{1}$ cell cycle arrest to allow time for DNA damage repair. Moreover, these events are believed to precede development of basal cell and squamous cell carcinoma and occur in the early stages of malignant melanoma. Taken together, research findings indicate that p53/p21 expression could potentially serve as a useful marker for DNA damage, allow for the diagnosis between normal versus chronically UV-exposed skin and serve as a predictive indicator for the onset of malignant melanoma.

In line with this, the major aim of the present study is to test the effectiveness of milk phospholipids' protective properties against UV-induced photodamage by evaluating p21 protein expression, hyperplasia and apoptotic markers in in vitro human skin equivalent tissues using Western blot and fluorescence and immunofluorescence microscopy. The purpose of which is to shed light on some basic molecular mechanisms behind previously observed changes in tissue morphology that have demonstrated a positive correlation between 
milk phospholipid treatment and reduction in UV-induced photodamage (Russell et al. 2010). Further developments of the study may eventually have implications for early detection, prevention and possible development of targeted therapy against UV-induced DNA damage and skin cancer. 


\section{Chapter II. Background}

\subsection{Human Skin Structure and Function}

The skin is the largest organ of the body accounting for around $10 \%$ of our total body mass and covers an average of $1.7 \mathrm{~m}^{2}$ of our body. It principally serves as a protective covering, while its multiple layers provide diverse functions critical for survival. The two major regions of the skin are the dermis and epidermis. The dermis is a vascularized tissue, whereas the epidermis contains no blood vessels and hence, receives nutrients by relying on diffusion across the dermal and into the epidermal layer. A layer of loose connective tissue containing mostly areolar and adipose tissue lies beneath the dermis and is known as the hypodermis, subcutaneous layer or superficial fascia.

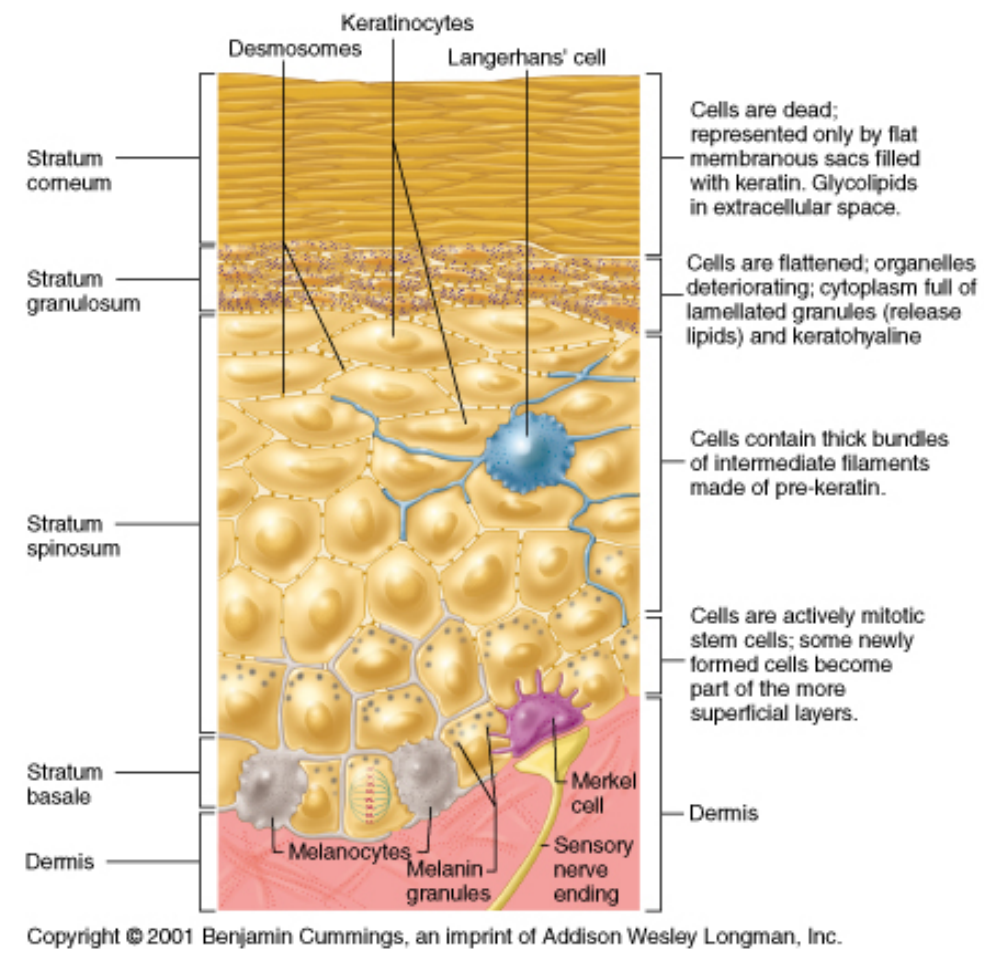

Figure 2. Layers of the epidermis. Source: Cummings, (2001).

The outermost layer of the skin is the epidermis. Its average thickness is about 0.2 millimeters and has a maximum thickness of 1 millimeter. It is estimated that $0-10 \%$ of $300 \mathrm{~nm}$ wavelength radiation is easily transmitted through the epidermis into the dermis. The epidermis is composed of four types of cells which include keratinocytes, 
melanocytes, Langerhan cells and Merkel cells. Keratinocytes constitute 95\% of the epidermal layer and are basically cells that synthesize fibrous proteins known as keratin. Production of these proteins is under the control of hormones called epidermal growth factors. Melanocytes on the other hand, can be found in the deeper layers of the epidermis and produce the pigment melanin that gives a brown color to skin and hair. Their primary function is to protect deeper skin tissues from some of the harmful effects of solar radiation by serving as an energy sink through the absorption of radiation and suppression of free radicals (Williams, 2003). Merkel cells are cells associated with nerve endings in the basement membrane and are therefore plenty in regions with enhanced sensitivity such as the lips and fingertips. Langerhans cells on the other hand, are derived from the bone marrow and play an immunologic function by presenting foreign antigens to lymphocytes in the lymph node.

The five strata making up the epidermis are the stratum corneum, stratum lucidum, stratum granulosum, stratum spinosum and stratum basale. The stratum corneum or the horny layer consists of corneocytes, which are flat squamous cells, ranging from 10-30 $\mu \mathrm{m}$ in size as seen through a confocal microscope. Corneocytes are the final outcome of epidermal cell differentiation and are characterized as non-nucleated, dehydrated and keratinized (cornified) dead cells embedded in a lamellar lipid matrix separated by interphases or contact junctions between two different phases. Cells that make up this layer have a heterogeneous surface with robust outer membrane and act as the main barrier to transdermal drug delivery (Williams, 2003). The stratum corneum is about 15 microns thick with around 10-15 cell layers. In Caucasian skin, the layer transmits about 17-66\% in the sunburn UV range of 290-320 $\mathrm{nm}$ (UVB). The translucent layer that lies beneath the stratum corneum is the stratum lucidum or the clear layer, but is considered by some as part of the lower layers of the stratum corneum. It is in this layer that the nucleus completely disintegrates and where further keratinization and flattening of cells occur. Oil droplets that are sometimes observed within this layer correspond to disintegration of lysosomes.

Underlying layers to the stratum corneum are referred to as the "viable epidermis", which may not be applicable to stratum granulosum since degradation of cell components in this layer accompanies their differentiation. Granular cells are about $25-35 \mu \mathrm{m}$ in size and are found at average depths of $15-20 \mu \mathrm{m}$ below the stratum corneum. They form the stratum granulosum layer, which is a very thin layer (1-3 cell layers thick) and is sometimes absent in thinner regions of skin. These cells are so called because grainy structures are seen within their cytoplasmic region, which correspond to organelles and melanosomes. They are believed to be responsible for the 
reflectance and white tone of the skin. These cells arise as mature, flattened keratinocytes from the stratum spinosum layer and possess enzymes that begin to degrade their nuclei and organelles. Keratohyaline granules are responsible for the maturation of keratin within these cells.

Stratum spinosum and stratum basale together make up the Malphigian layer. Maturation of cells in the stratum basale gives rise to a layer containing mostly keratinocytes, the stratum spinosum or squamous cell layer. This is the thickest layer of the epidermis comprising up to six rows of columnar to polygonal cells in the order of proximity to the surface. These cells generate keratin, which renders the skin, hair and nails tough and flexible. Spinous cells are about 15-25 $\mu \mathrm{m}$ in size and can be found at about 20-100 $\mu \mathrm{m}$ depth. There is a decrease in cell and nuclear size observed as viewed from the superficial to the deeper spinous layers, yet the nuclear to cytoplasm ratio increases along with the cell density and melanin content.

Finally, the stratum basale or stratum germinativum, as aptly termed, contains the only layer of stem cells or mitotically active keratinocytes. They are simple columnar or cuboidal in shape and form the deepest layer of the epidermis. They constantly proliferate, generating one daughter cell that remains in the basal layer and one keratinocyte that differentiates and eventually pushes and replenishes skin shed from the uppermost layer. The surface then sheds off at a rate of about once every two weeks. It is also comprised of about $25 \%$ melanocytes as well as Merkel cells and Langerhans cells. In general, basal cell clusters, when viewed horizontally (en face) on a confocal microscope, are arranged in a single layer located on the dermal papillae at $100 \mu \mathrm{m}$ deep. Each cell is about 7-12 $\mu \mathrm{m}$ in size (Rajadhyaksha, Gonzalez et al. 1999).

At the junction between the epidermis and the dermis lies a thin layer known as the basement membrane or dermo-epidermal membrane. This membrane is attached to the basal layer by protein anchors and hemidesmosomes, which when compromised result in shedding of the skin such as those observed in blisters. The basement membrane contains structural and signaling proteins such as collagen IV, laminin, collagen VII and integrin $\alpha 6$ that can be visualized through immunohistochemical methods. This layer acts as a physical and separation barrier and attachment layer for the epidermis and dermis. It is of critical importance during cancer metastasis since initial invasion of skin cancer into deeper tissue involves breaching through this layer.

The dermis, also called corium, has main components that include collagen, elastic fibers, ground substances, nerves, capillaries, hair follicles and sweat glands. Its total thickness ranges between 3-5 $\mathrm{mm}$ and is the 
major component of the human skin (Williams, 2003). The network of connective tissues in the dermis contains mostly collagen fibrils, which offer structural support to the skin, while the elastic tissues impart flexibility. Both structures are embedded in a mucopolysaccharide gel matrix (Wilkes et al. 1973) and are largely considered as relatively minor barriers to the topical delivery of agents with hydrophilic nature, but significantly impede lipophilic molecules. A wealth of interconnections can also be found within the dermal matrix. These include blood vessels, lymphatic ducts, nerve endings, hair follicles, sebaceous glands and sweat glands. In horizontal sections of the dermis in vivo, capillary loops are reported to be visible within each papilla at average depths of $100-150 \mu \mathrm{m}$.

The hypodermis is a subcutaneous fat layer that interconnects the dermis to the tissue underneath and carries the blood vessels to the skin, which reaches to within $0.2 \mathrm{~mm}$ of the skin surface, while intertwined with the Malpighian layer of the epidermis. Its thickness reaches to several millimeters in most regions of the body except the eyelids. The adipose tissue present in this layer provides insulation, protection from physical shock and energy supply.

The human skin performs diverse functions ranging from thermoreception, protection, excretion, immunity, blood reservoir and site of Vitamin D synthesis. The human skin's unique adaptive characteristics are fundamental for successful subsistence in terrestrial environments, through the regulation of body temperature and water loss, while providing a barrier against corrosive or toxic agents and microorganisms. Heat regulation is attributable to the extensive vascular network of the skin. The blood vessels are also responsible for the transport of oxygen and nutrients, removal of waste products and toxins and delivery of immune factors during wound repair. The lymphatic vessels play a role in maintaining interstitial pressure and facilitate transport of waste and immunologic agents during infection.

\subsection{Experimental Model Systems}

Development of well-characterized skin models that closely mimic the physical, metabolic and physiological properties of skin in vivo are highly in demand for toxicity testing, studies implementing safety standards and in research dealing with basic skin biology, wound repair, melanogenesis, skin disease pathogenesis 
and skin cancer. The choice of skin testing model is crucial in establishing the legitimacy of skin care research findings and the strength of consequent assumptions deduced from them.

\subsubsection{Murine Models}

Much research has been carried out to study UV carcinogenesis in mouse skin, with the ultimate goal of correlating these results to the biological responses in human skin. Many consider mouse skin as a standard model in studying the molecular mechanisms behind the initiation, promotion and progression of carcinogenesis. Characteristics of mice that many deem appropriate for testing variables in photobiological studies include their small size and ease of handling in relatively high numbers. Although the general structure of skin is conserved between species, caution is often necessary when extrapolating experiments on animal models to human cases due to other inherent differences between species in terms of hair distribution and growth, pigmentation, vascular networks and inflammation. For example, quantitative data collected from mice studies on the amount of ultraviolet light needed to induce cancer would be invalid when translated to its carcinogenic effects on humans since human skin has much greater thickness and hence, deeper layers are harder to penetrate. Nonetheless, mouse skin cancers induced by UV display similar p53 mutations present in human skin cancers (Kanjilal et al. 1993; Ananthaswamy et al. 1997; Kress et al. 1992), although the specific exons involved and frequency of mutation may differ depending on the strain. Albino and hairless mice are often preferred subjects since the hair-growth cycle greatly affects the reaction to physical, chemical and photoactive agents.

Three main reactions for the observed action spectra of UV radiation in mouse skin include delayed edema, which is the counterpart for erythema in human skin; hair growth inhibition, which occurs only in the early anagen or the active hair follicle growth phase of the cell cycle; and immediate edema. In delayed edema, the most effective UV wavelength is $260 \mathrm{~nm}$ during early anagen phase and $290 \mathrm{~nm}$ in mid-anagen phase. Hair-growth inhibition demonstrates a single wavelength peak at $290 \mathrm{~nm}$. Lastly, immediate oedema has a maximum effect at $300 \mathrm{~nm}$. The most probable cause of the delayed edema is believed to be rupture of the lysosomal membrane by lipid peroxides produced upon exposure to UV radiation and the consequent release of hydrolytic enzymes. Similarly, hair growth inhibition is attributed to lysosomal damage in hair germ cells. In contrast, the release of histamines from few and 
scattered mast cells within the dermis accounts for the immediate edema reaction. In mice with the mutant gene, rhino, chronically exposed to radiation, inflammatory response has often been noted to occur prior to tumorigenesis, which frequently results in squamous cell carcinomas. In both hairless and haired mice, reddish-brown discoloration of skin are usually observed 48 hours after irradiation with 500 to $900 \mathrm{~mJ} / \mathrm{cm}^{2}$ UVB dose, and this threshold for UVR erythema is very near that of skin ulcerations. However, no erythema reactions resembling that of human skin were observed at any time. Therefore, data relevant to the usual sequence of skin changes in human skin cannot be extrapolated from the biological response of mouse skin.

Similarly, UV carcinogenesis studies in mice gave way to some important insights such as findings that sarcoma (connective or soft tissue cancer) are predominantly induced by radiation above 280 nm, whereas the more superficial epidermal carcinoma are induced by monochromatic radiation at $254 \mathrm{~nm}$. In comparison, a correlation of this action spectrum is seen with human skin, in that dermal reactions are observed with doses of about $300 \mathrm{~nm}$ but not with $260 \mathrm{~nm}$.

In terms of single exposure to UV radiation however, rhino mice exhibit erythema response patterns that closely approximate that of humans (Urbach, 1969). Rhino mice develop greater epidermal thickening than hairless mice, which in effect, protect deeper dermal vessels from direct radiation, while inducing indirect erythema response. Dilation of dermal vessels largely triggers the general erythema response observed. For this reason, the rhino mice are considered most suitable among the murine models for photobiological research of erythema, inflammation and carcinogenesis although it is still wise to exercise prudence on their applicability to humans due to observed differences in their pigmentation characteristics.

\subsubsection{Guinea Pigs}

There are several reasons for using guinea pigs for photobiological studies. Irradiation of guinea pigs results in biological reactions with sequence and time intervals that closely mimic human reactions (Urbach, 1969). These animals are also preferred for enzyme assay studies for sunburn through the withdrawal of blood and measurement of the enzyme levels such as plasma beta glycuronidase. Guinea pigs have also proven to be excellent experimental models for testing topical and systemic chemicals that induce photosensitivity at greater than $320 \mathrm{~nm}$ 
wavelengths. The main drawbacks for using guinea pigs however, include their poor response to skin cancer studies involving UV light, high purchase and maintenance costs and the need for hair removal procedures.

\subsubsection{Cultured Cells and Cell Lines}

Cell-based systems for experiments are usually pure monolayer cultures or co-cultures of human and animal skin cells, especially keratinocytes. But several types of organisms and animal cells have also been studied to determine the effects of UV irradiation on specific cell activities. Among commonly cultured cells include protozoans, gametocytes, blood cells and neurons. Alternatively, cancer cell lines provide in vitro systems for investigating elaborate mechanisms as well as serve as testing systems for skin therapeutic agents.

Protozoans are chosen for the ease of synchronizing their cell cycles for a specific study. Most commonly used protozoans for UV studies are ciliates and Amoeba, with the latter owing to the ease of separating their nucleate and anucleate regions as well as transplanting one cell's nucleus to another. It was also from the study of UV effects on protozoans that revealed an increase in UV sensitivity observed with starved cells, which may be explained by the nucleic acids being in a more open conformation to direct exposure or to the presence of UV protective compounds in the well-fed state (Giese, 1938; Giese and Reed, 1940).

The gametocytes or eggs and sperm of marine invertebrates, especially of sea urchins, are also preferred experimental subjects for the study of the effects of UV on cell division. This is attributed to their inherent nutrient reserves and the synchronous manner of cell division between different cells, thereby offering a practically homogeneous sample population.

Blood cells, on the other hand, are readily available for sample sizes of up to millions and several UV and permeability studies have been conducted using these cells. However, it is crucial to use media that is free from UV absorbing substances since, aside from the pigment hemoglobin, serum proteins are able to absorb short UV wavelengths and thus, inadvertently give protection to the cells. For this reason, salt solutions isotonic to the cells and which absorb negligible radiation are often utilized to suspend the cells during UV treatment

Tissue cultured cells easily adhere and propagate on culture surfaces and are supplied with nutrient growth medium and growth factors. They are highly favorable for studies that involve manipulations at the molecular level 
including recombinant DNA technology and gene and antisense therapy. Synchronization at a particular stage of the cell cycle is also possible with a double-thymidine block and release protocol (Shedden and Cooper, 2002; Hengst and Reed, 1996). As mammalian cells are increasingly utilized, the outcomes derived from these experiments can be expected to be a lot more relevant to humans.

Melanocytes are recently being selectively cultivated from newborn and adult skin due to common cases of overgrowth and invasion form other skin cell types such as keratinocytes and fibroblasts (Gilchrest et al. 1984). The inclusion of serum to their growth medium components limit their usefulness in studies that involve substances having functional similarities to serum factors that mask their inherent properties (Abdel-Naser et al. 2005). These cells offer high reproducibility however, due to the amenability of melanocytes to numerous passaging up to 12-14 passages (Eisinger and Marko, 1982). To further increase the validity of experiments, they can often be co-cultured with keratinocytes to approximate their actual interaction in vivo since keratinocytes reportedly generate factors that participate in melanocyte function and growth (Abdel-Naser et al. 2005). Specific effects of UV on melanocytes include induction of their melanization and inhibition of their proliferation. UVA evokes no substantial effects on melanocytes unlike UVB, which has been known to directly activate cultivated melanocytes. Furthermore, human melanocytes are often utilized as controls for melanoma cell research.

Epidermal keratinocytes are grown purely using serum with DMEM/Ham's F12 and a feeder layer of irradiated, transfected mouse 3T3 cells. Their pattern of growth is that they usually attach as single cells or small clusters, and then grow around the colony, stratifying in the center until they form coherent colonies or multi-layered sheets (Rheinwald et al. 1977). Their cell cycle is about $22 \mathrm{~h}$ and therefore, the cells tend to hyperproliferate. They are thus beneficial for skin grafting and keratinocyte grafting for burns, ulcers, nevi excision sites and hypospadia correction. Employment of keratinocytes has also led to the understanding of numerous growth and differentiation factors, as well as provide ideal hosts for viral and oncogene transfection studies and anti-sense therapy.

Dermal fibroblasts are very abundant in the loose connective tissue playing major roles in the synthesis of the tissue components and precursors, including collagen and elastin of the extracellular matrix. They are considered the easiest cell types to grow in vitro due to their low maintenance requirements and are, in fact, ubiquitously used as controls for virtually all biological research work. Their replicative capacity is up to about 50 - 100 doublings. They are also known to promote keratinocyte proliferation resulting in the hyperproliferation observed in psoriasis. 


\subsubsection{Ex Vivo Skin Models}

Ex vivo skin models or skin explants are often pieces of skin obtained from humans or animals for in vivo testing applications and are certainly advantageous owing to their easy accessibility. A great deal of research has been performed in screening for irritants, corrosives and determining the mechanics of dermal absorption or skin penetration. Mouse skin integrity function tests, human cadaver skin, surgical biopsies and pig ears (Zuang et al. 2005) are some of the specific samples used in testing skin irritation. In regards to the photobiological effects of UV on human skin, ex vivo studies that have been conducted include sunburn reactions and carcinogenic effects of UV. Human skin biopsies are employed in studies due to experimental and ethical complexities associated with alternative testing systems, which may or may not be constructive since it causes simplification of the system such that normal skin cell regeneration halts, immune responses no longer interfere and metabolic activity is lost.

\subsubsection{Human Skin Equivalent Tissue}

Local skin reactions to harmful agents very seldom involve systemic biological responses. For this reason, it is acknowledged that local skin reactions may be simulated and predicted by in vitro model systems that only closely approximate skin barrier properties and reactivity observed in vivo. A current leading technology alternative to animal testing that is actively being sought by current industrial and toxicology laboratories is the human skin equivalent culture model. Tissue engineered skin is often cultured to replicate many features of the human skin. Multi-stratified epithelial architecture is formed underneath a functional stratum corneum and the epithelial cells

possess same differentiation characteristics as that of the native epidermis. A robust functional barrier identical to that found in natural skin surface is also present in these models provided their multi-layered stratum corneum contains the essential lipid profile (European Centre for the Validation of Alternative Methods [ECVAM], 2009). Co-cultured skin consists of a mixture of two or more different cell types present in skin and more recent advancements have paved the way to optionally develop engineered tissues with both dermal and epidermal layers.

There are several other advantages associated with employing three-dimensional skin equivalents over other skin models. They are cost-effective and circumvent ethical, social, political and legal concerns associated 
with animal testing. Its distinct advantage against human skin biopsy is that tissues remain metabolically active and as a result, skin regeneration may not completely stop while in culture. Active mitosis and metabolism similar to living tissue generally permits an increased accuracy of findings. Since tissues are grown at the air-liquid interface, topical application to the surface of the stratum corneum can be easily done and effectively mimics this route of human exposure. In this way, water insoluble chemicals prevalent in most skin formulations can be tested without having to dilute them into solvent media. The uniformity of engineered tissue's thickness also permits greater reproducibility and allows steady state diffusion rates to be reached faster compared to cadaver skin. Plus, physiological interactions between the dermis and epidermis can be studied. And lastly, other clinically relevant skin properties such as transepidermal water loss barrier effects and hydration can be tested. A constantly increasing volume of data obtained from studies that use three-dimensional human tissue models is proof that they are deemed useful for in vitro studies of skin related issues.

Various tissue types with similar in vivo morphology and growth behavior have been developed and can be purchased from different vendors. Examples of tissue models that have been recently validated by alternative methods and regulatory authorities (i.e. ECVAM, ICCVAM, ESAC and OECD) for assessing not only skin irritation or corrosion but also the efficacy of tested products, include EpiSkin (Skin Ethic Laboratories, Lyon, France), EpiDerm (MatTek Corporation, Ashland, MA), VitroLife-Skin (Gunze Medical Devices Limited, Kyoto, Japan), LabCyte EPI-MODEL 24 (Japan Tissue Engineering Co., Ltd., Aichi, Japan) and EST-1000 (CellSystems, St. Katharinen, Germany).

\subsection{UV Radiation and its Immediate Effects}

Light exists in a particle-like state as packets of energy (quanta) known as photons, as well as in a state that behaves like a wave. This property is known as wave-particle duality in quantum physics. Planck's formula, $E=h f$, expresses the directly proportional relationship between energy, $E$, in one quantum and the frequency, $f$, of the corresponding waveform. The constant of proportionality, $h$, is called Planck's constant. In quantum terms, there are different types of radiation depending on the amount of energy associated with a single quantum and the electromagnetic spectrum describes the entire range of these different energies. 
Sunlight is a continuous spectrum of electromagnetic radiation that falls within the ultraviolet (UV), visible and infrared wavelengths. UV radiation possesses one of the highest energy quanta (200-400 nm) next to X-rays and gamma rays and is immediately followed by visible light $(400-700 \mathrm{~nm})$. Fortunately, the ozone layer shields us from radiation with wavelengths of $290 \mathrm{~nm}$ and below.

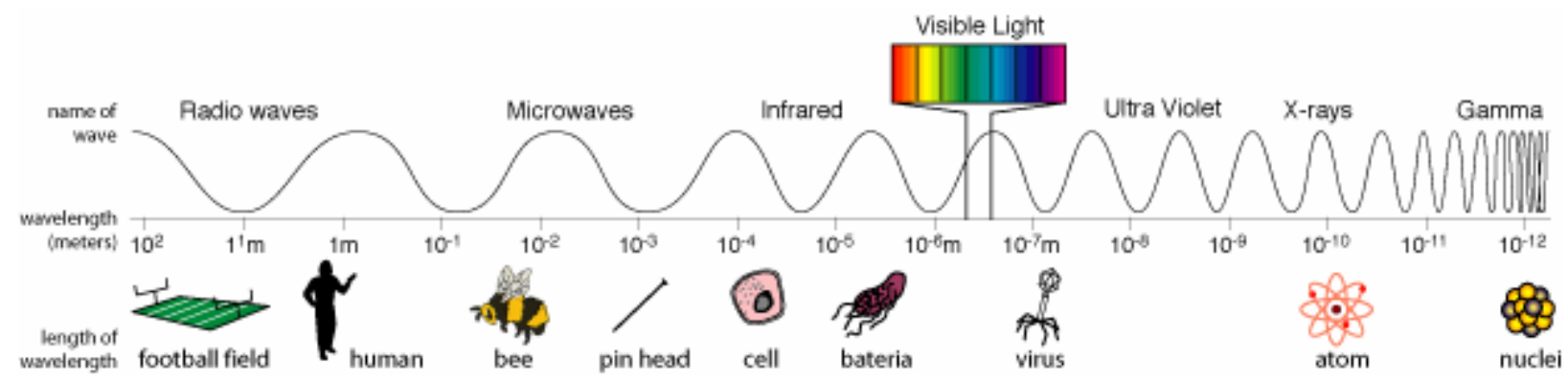

Figure 3. Electromagnetic spectrum showing the relationship of wavelengths from radio to gamma waves (n.d.).

Source: www.nasa.gov

Several Laws of Photochemistry relating the interaction between light and matter need to be taken into account when investigating the biological effects of radiation. Grotthuss-Draper Law or the Photochemical Absorption Law states that only absorbed radiations are capable of successfully inducing photochemical effects. Consequently, reflected, scattered, fluorescent and transmitted light have indirect roles, if any, in UV radiation's biological effect on skin tissue. The wavelength of the incident radiation can be indicative in determining the absorption spectra of the tissue or in essence, which particular fraction of light is absorbed. Moreover, only a percentage of light that is absorbed participates in a specific photochemical reaction. This is known as the quantum yield and is expressed as the number of molecules affected over the amount of absorbed quanta. In practice, varying wavelengths do not influence the quantum yield which generally remains constant. Rather, it is mostly dependent on the absorbed radiation than on the incident radiation.

Reciprocity Law states that when the product of the intensity and the exposure time is constant, the photochemical effect is the same. From this, it can be deduced that the total energy, as opposed to the dose or the duration of exposure, greatly determines the extent of the photochemical effect. Thus, exposure to solar radiation has a cumulative effect and a single high intensity exposure may be comparable to low dose chronic exposure. StarckEinstein Law or the Law of Photochemical Equivalence states that when one quantum of light is absorbed per molecule of absorbing substance, one light-activated molecule is produced. However, it is also apparent that not all absorbed light is involved with a specific photochemical reaction. 
The portion of sunlight that induces the most biological damage is known as ultraviolet radiation (UVR) and is arbitrarily divided into UVA, UVB and UVC, which comprise the UV spectrum. UVC radiation has the shortest wavelength $(200-280 \mathrm{~nm})$ and the most intense energy among the three. For this reason, it is capable of causing the most severe damage to biological tissue. Luckily, UVC radiation does not reach the earth's surface due mainly to the effective shielding of the ozone layer in the atmosphere, although these radiations are also artificially produced for germicidal lamps.

UVB and UVA on the other hand, both reach the earth's surface and have greater biological significance due to the amount and frequency of exposure of our skin and eyes. UVB makes up 1-10\% of solar radiation reaching the earth surface, ranges from 280-320 nm and is mostly absorbed in the epidermal region (Parrish, 1983). DNA readily absorbs UVB and overexposure is believed to be responsible for most of the actual photodamage to human skin. UVB is also known to be a complete carcinogen capable of both initiating and promoting non-melanoma cancers (Bowden, 2004). Then again, dense clouds, tight-knit clothing and glass window panes easily block radiation within this wavelength range. Furthermore, moderate exposure to UVB (around 15 minutes a day) is essential for the production of vitamin D from sterols, while a small amount of visible sunlight exposure is known to boost a person's psychological health.

UVA radiation consists of $90-99 \%$ of solar radiation that reaches the earth surface and has the longest wavelength ranging between 320 to $400 \mathrm{~nm}$. It penetrates deepest into the skin up to the subcutaneous tissue (Figure 4) and can even affect circulating blood cells (Moller et al. 2002). A noteworthy difference between UVA and UVB is that UVA has less effect on the cell cycle than UVB in epidermal cells (de Laat et al. 1997). UVA is also a weak carcinogen with its erythema and direct DNA damage effects a thousand-fold less potent than UVB's (2010, Three types of ultraviolet radiation section, para. 3). Although UVA does not induce sunburn, DNA damage does result deep within the skin tissue, which may develop into mutations and increase the risk of skin carcinogenesis (Rajadhyaksha et al. 2001; Rebel et al. 2001). 


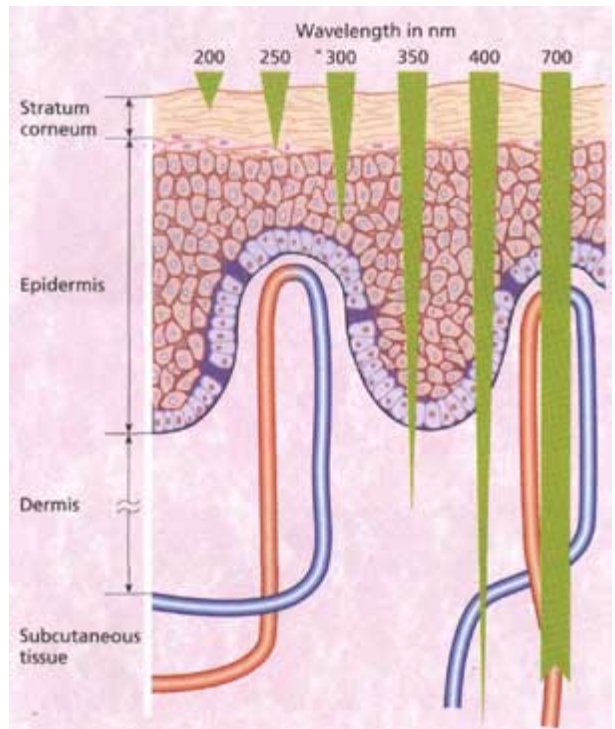

Figure 4. Skin penetration depth of ultraviolet radiation of different wavelengths. Source: pgbeautygroomingscience.com, (2010).

However, UVA is more potent in advancing tumorigenesis due to its capacity to induce more oxidative stress than UVB (Tyrrell, 1991). Specifically, UVA is 10 times more efficient at inducing lipid peroxidation (Morliere et al. 1995), is more cytotoxic (de Gruijl et al. 1994), induces DNA strand breaks and nucleic acid oxidation (Wenczl et al. 1997), inhibits DNA repair (Parsons and Hayward, 1985) and promotes matrix metalloproteinase (MMP) synthesis (Fisher et al. 2001) - all of which enhance skin cancer aggressiveness. There is also evidence that UVA not only causes photoaging but also skin cancer in animals at high doses and for repeated exposures over a long period (Zigman et al. 1976; Strickland, 1986). On top of these, long wavelength UVA radiation penetrates into clouds, loose clothing and untinted glass easily and is much less susceptible to atmospheric scattering and absorption.

\subsection{Biological Effects of Solar UV Radiation}

The biological response after UV irradiation is rather complex and is still in the process of being completely elucidated. Add to this the variety of energy sources that are used in studies which compounds the difficulties in documenting the precise effects of UV on tissue processes. Most of the absorbing material such as keratin or melanin may be chemically inactive, while energy transfer may also occur between neighboring molecules. A trough at $280 \mathrm{~nm}$ of the UV action spectra is believed to be caused by absorption of inactive keratin. 
Overall however, UVB exposure of skin results in biological responses via direct DNA damage or the formation of free radicals that damage target biomolecules, especially DNA (Matsumura and Ananthaswamy, 2002). Erythema and pigmentation have long been considered primary determinants of the biologic reaction at the tissue level despite individual and seasonal variations and lack of established details on concrete mechanisms that bring about these reactions. In addition to these, other general biological changes observed upon irradiation include premature keratinization, shedding of the epidermal surface, infiltration of leukocytes, mitosis and cell proliferation.

\subsubsection{Effects of UV on Tissue Morphology}

\subsubsection{Erythema}

Acute overexposure of the skin to sunlight has consistently been observed to result in sunburn, which is a manifestation of apoptotic epidermal keratinocytes (Danno and Horio, 1987). It is now believed that cell death response is critical in preventing skin cancer (Brash, Rudolph et al. 1991). Among the most established and highly studied sunburn responses is the appearance of erythema, which is a term for the intense reddening of the skin as a result of the increase in blood flow through dilated blood vessels at the dermal layer. More specifically, this develops from an increase in permeability of arterioles and venules in the corium. There is usually a latent period between the appearance of erythema and UV exposure, which can be attributed to the diffusion of molecular effectors. The mediator molecules responsible for the changes in vascular behavior are said to be produced by damage to the epidermal cells (Blum, 1955), possibly triggering the release of hydrolytic enzymes from the lysosomes as observed in vitro (Weissmann, 1961).

The reciprocal of the threshold dose values for erythema is considered to be the basis of the biologic action spectrum in Caucasian skin (Figure 5). The characteristic peaks of absorption at these wavelengths correspond to the formation of erythema. Previous studies have shown that the appearance of erythema follows a variable time course dependent on the wavelength (Urbach, 1969). Both visible and long wavelength UV radiation show good transmission in erythema-prone regions of the skin. The highest percentage of incident UV transmission and absorption into bloodless skin is observed at UV wavelengths below $320 \mathrm{~nm}$. More precisely, the highest peak of 
effective wavelength is around $280 \mathrm{~nm}$, but a lower maximum peak value is evident at 250 nm (not shown). The charcterisitic peaks of absorption at theses wavelengths correspond to the formation of erythema. However, there is a drop in the erythema effectiveness curve toward shorter wavelengths. There is also a decrease in light intensity at greater penetration depths, which is accounted for by true absorption as well as scattering in the opaque skin medium. Hence, a drop in erythema effectiveness toward shorter wavelengths is presumed to be an effect of the inability of shorter wavelengths to penetrate deeper sensitive regions of the skin tissue adequately due to high absorption at shallow depths.

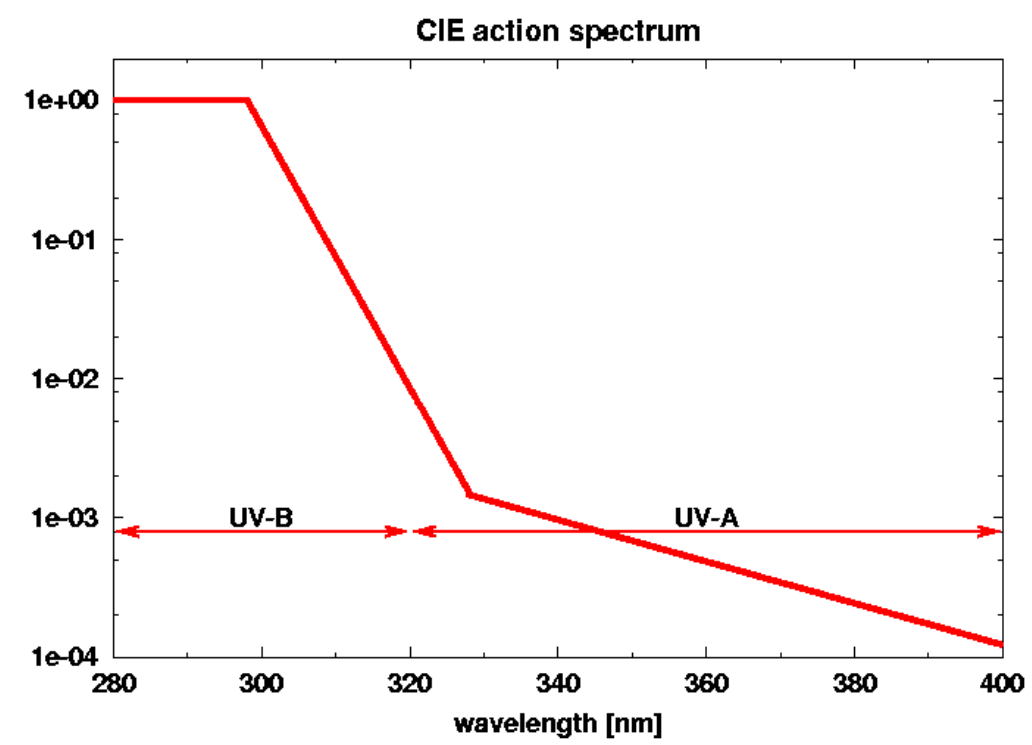

Figure 5. CIE action spectrum for the susceptibility of the Caucasian skin to erythema. Source: KNMI / TEMIS, (2006).

Around the $254 \mathrm{~nm}$ wavelength is where the lowest amount of energy is required to produce a minimum perceptible erythema, yet maximum erythema and tissue destruction is very hard to achieve at this wavelength even at multiple doses. In contrast, wavelengths of about $313 \mathrm{~nm}$ require at least 100 times the energy that induces a minimum perceptible erythema, but merely $10 \%$ above this threshold dose results in maximal erythema and consequently, blistering. Therefore, the carcinogenic properties of these wavelengths also vary makedly, so it would be unwise to assume that the behavior of the different wavelengths are additive when choosing the appropriate wavelength for studying the effects of UV on skin. 


\subsubsection{Pigmentation}

One of the important risk factors in the development of skin cancer is the extent of skin pigmentation and the overall ability to tan (Soehnge, Ouhtit et al. 1997). People with minimal skin pigmentation who sunburn easily and suntan poorly are at the highest risk for non-melanoma skin cancer (Gloster and Brodland, 1996). Sunlight induces growth and density increase of melanocytes, production of melanin pigments and an increase in the transfer of melanosomes to keratinocytes, which in effect, confer immediate protection to the nuclei of skin cells. Melanogenesis is believed to be induced most effectively by radiation within the erythemal spectrum $(<320 \mathrm{~nm})$ (Blum, 1955) and less so by wavelengths between $320-700 \mathrm{~nm}$. This radiation comprises more than $50 \%$ of the solar radiation that reaches the earth's surface (Pathak et al. 1962). The biological responses induced in erythema response also lead to melanogenesis and increased pigmentation. In pigmented skin, melanin granules or melanosomes are more abundant, more electron dense and localized in deep clusters as opposed to fair skin. Skin exposed to solar radiation at noon in the summer season experience vitamin D production from sterols, all within 1020 minutes upon exposure. After a relatively high intensity exposure, "late" pigmentation and blistering can be observed after 2-3 days. In addition, pigmentation can be induced by DNA damage, specifically, single stranded DNA and thymine dimer products (Eller et al. 2000).

Currently, there are two mechanisms of melanin formation that have been thoroughly studied. One is the immediate pigment darkening known as Meirowsky phenomenon and the second is the formation of new pigment or melanogenesis, which occurs in a three-part process. In immediate pigment darkening, the pigment is preformed but exists in a colorless or a reduced state, which darkens maximally after radiation exposure without any latent period between exposure and darkening. This is followed by gradual fading of the darkened region to almost undetectable levels within 3-4 hours. Black coloration is attributed to the oxidation of melanin that generates a semiquinone-like free radical, while brown coloration results from the reduction of a melanin polymer. The former occurs as a reversible oxidation-reduction reaction and the free-radical rapidly decays after withdrawal of radiation.

Melanogenesis in contrast, starts with the formation of an erythemal response such as sunburn, followed by melanin production, then migration of melanosomes to the keratinocytes. In older individuals, solar lentigines or 
regions of skin damage with higher number of melanocytes and increased melanin production form as a result of cumulative sun exposure in the area.

\subsubsection{Autofluorescence}

Autofluorescence is a distinctive attribute of the skin tissue. Natural fluorophores in the skin are capable of absorbing short wavelength light (i.e. UV, violet or blue) and emitting light of longer wavelengths. Native fluorophores in the skin include: tryptophan, collagen fibers, elastin, reduced nicotinamide adenine dinucleotide (NAD), flavin adenine dinucleotide (FAD), and protoporphyrin IX (Katz and Alfano, 1996; Schomaker et al. 1992; Sackman, 2000). The most notable ones however, are keratin, dermal collagen and NAD. Studies have shown that diseased and healthy ex vivo skin tissue have different autofluorescence profiles when excited at $365 \mathrm{~nm}$ and detected for autofluorescence at $465 \mathrm{~nm}$ (Delgado et al. 2002). However, an entirely different trend from erythema production governs wavelength dependence in skin tissue fluorescence such that maximum fluorescence occurs at $366 \mathrm{~nm}$ wavelength where erythema production is practically absent (Urbach, 1969).

\subsubsection{Immune Suppression}

Solar radiation, especially UVB at low levels of at least 1 minimal erythema dose (MED) or the minimal single dose of UVR required to produce erythema, can trigger immunosuppression in about $40 \%$ of individuals and all basal and squamous cell carcinoma patients (Streilen et al. 1994) and hence, further promote skin carcinogenesis by allowing neoplasms to escape recognition and rejection. It has been reported that normally, most UV-induced mouse skin tumors are rejected when transplanted to normal syngeneic hosts but not in mice exposed to subcarcinogenic UV doses (Kripke, 1981). Contact hypersensitivity (CHS) response normally initiated by Langerhans cells is also inhibited at locally exposed sites (Streilen et al. 1994). Cytokines such as TNF- $\alpha$ (Yoshikawa and Streilen, 1991), interleukin (IL)-10, IL-1 (Robertson et al. 1987) and other factors (Luger and Schwarz, 1990) are produced by keratinocytes upon irradiation and have been linked to the immunosuppression observed in these cells. Other phenomena believed to occur are the surge in the levels of suppressor T-cells in 
response to damaged Langerhans cells and skin infiltration of macrophages during UV-induced inflammatory response. Still another probable cause is the keratinocyte's release of soluble factors or cytokines such as IL-10, TNF- $\alpha$ and ILF- $\alpha$ into damaged sites, as an immediate response to UV damage (Ullrich, 1995). These compounds serve to induce immunosuppression or hamper T-cell mediated responses including the response that rejects skin tumors. Furthermore, chronic exposure of mice to UVB induced defects in antigen presentation in cutaneous cells (Bacci et al. 1996) although this is reversible during photosome-mediated DNA repair of dimers (Vink et al. 1997). Antioxidant treatments however, are capable of protecting against immunosuppression (Duthie et al. 1999).

\subsubsection{Photoaging}

Chronic exposure to UV results in the gradual accumulation of photodamage to skin which only becomes apparent after a few decades. This process depends highly on the skin type and the total amount of time of exposure. Since most UVB is absorbed in the epidermis, photoaging responses can be attributed to UVA alone (Pinnell, 2003). Yet the changes described below are very unlikely to be perceived in individuals spending their lives mostly indoors. In essence, the epidermis becomes thicker as a whole, deeply wrinkled, dry and leathery in appearance. Epidermal cell degeneration at the region of UV absorption in the upper epidermis is considered a direct effect of UV exposure whereas glycogen accumulation in the basal cell layer upon sunburn is believed to be an indirect effect of UV exposure. In contrast, the stratum corneum increases in surface area but gets thinner with age. At the cellular level, keratinocytes become irregular in size and shape and lose polarity; melanocytes accumulate in regions of increased or decreased numbers; and Langerhans cells decrease in actinic skin. The frequency of skin cell renewal is reduced and dermal blood vessels become dilated, bent or twisted, while capillaries begin to diminish in number. Among the dermal cells, the collagen stains as basophilic, become irregular and disorganized and glycosaminoglycans and deranged elastin tissue increase. Induction of MMP proteases by even a small amount of UV irradiation results in the breakdown of collagen in skin (Fisher et al. 1996) and inhibition of procollagen synthesis (Fisher et al. 2000). Chronic UV exposure over months will cause actinic elastosis, a degenerative change in the dermal connective tissue characterized by the replacement of collagen with a thickened mass of degenerated dermal elastic fibres. This believed to be caused by oxidative stress (Kawaguchi et al. 1997) and is often associated 
with, but not essential to human skin cancer formation (Sams et al. 1964). Eventually with extended periods of exposure, tissues within the periphery in the upper dermis turn blue-grey in color and become swollen. The small vessels then form red, highly fragile, spider-like branches termed telangiectases, which are easily damaged. Still in worst cases, actinic keratoses form as precancerous skin tumors appearing as crusty patches and predominantly develop in individuals who always burn and rarely tan in the sun (type I or type II skin).

\subsubsection{Hyperplasia and Epidermal Thickening}

UV irradiation has been shown to cause hyperproliferation in epidermal cells as well as in the deeper tissues, thereby leading to epidermal thickening caused by the formation of new stratum corneum. This response is considered to be an immediate defense mechanism resulting in an increased rate of cell renewal (Gonzalez et al. 1996). The phenomenon lasts for a few weeks before the skin returns back to its normal physiology in cases of low to moderate exposure. In contrast, severe exposure results in scarring, while chronic exposure is known to be a high risk factor at inducing skin cancer, which is essentially an uncontrolled and irreversible hyperplasia or abnormal increase in cell proliferation. Since the epidermis grows only in one dimension, the thickness of the layer is considered an appropriate gauge for actual cell number and cell growth. Epidermal thickening is at its maximum about one week, after which cell proliferation gradually declines. In repeated exposures however, as in UVB phototherapy, skin adaptation occurs by means of this process. As a result, the skin tissue experiences a reduction in its capacity to transmit UV light and somehow serves as a protective barrier to radiation. With subsequent exposures, the UV dose required to elicit perceptible erythema (MED) is increased by about $20 \%$ (Van der Leun and van Weelden, 1986). It has been hypothesized that a correlation between acute exposure-induced hyperplasia and cancers that appear with chronic exposure exists such that repeated induction of hyperplasia generates a deranged "run-away process" that may lead to carcinogenesis (Urbach, 1965). 


\subsubsection{Cellular and Molecular Effects}

Interactions between light and certain types of molecules such as molecules with aromatic groups and double bonds are more favored than others (Urbach, 1969). Thus, proteins, nucleic acids, porphyrins and carotenoids are particularly sensitive to light activation while carbohydrates and fats, except sterols like vitamin D and fat- and protein-rich membranes, are relatively inert. Among these candidate chromophores however, DNA and urocanic acid have been determined to have the highest biologic significance (Pinnell, 2003).

Absorption by amino acids depends on cellular environment factors such as $\mathrm{pH}$, temperature, solvents and the protein structure. In general however, the amino acids tryptophan, tyrosine, phenylalanine and cystine are capable of absorbing most near UV light at wavelengths $230 \mathrm{~nm}$ and above (McLaren and Shugar, 1964). In most cases, tryptophan fluorescence is observed after UV absorption. UV-induced enzyme inactivation has reportedly been due to either the direct destruction of amino acid residues in the active sites by light absorption or the damage of relatively weaker bonds (i.e. hydrogen bonds and hydrophobic interractions) as a result of energy transfer.

A critical concern is the high sensitivity of the DNA synthesis process to UV treatment and the relatively less sensitivity of RNA and protein syntheses as observed in bacteria and viruses (Kelner, 1953). This sensitivity of the DNA synthesis process to UV irradiation is primarily due to direct absorption by DNA bases of incident photons within the narrow UVB wavelength range, thereby resulting in thymine dimer formation between adjacent thymine residues in a single template DNA strand. In a similar fashion, absorbed energy in nucleotides can be transferred to

other molecules as in the case of thymine radical generation by energy transfer from adenine following UV irradiation of poly dAT molecules (Rahn et al. 1965). Nucleotide excision repair (NER) reverses UV damage and other DNA transcription errors in certain microbial species (Haynes, 1964) and some animal cells (Rasmussen, 1964) in nutrient-depleted media.

DNA easily absorbs UVB (290-320 $\mathrm{nm})$ and irradiation by UV light is known to denature nucleic acids by inducing changes between adjacent pyrimidine bases on one strand of DNA. Specifically, the changes lead to "signature" $\mathrm{C} \rightarrow \mathrm{T}$ and $\mathrm{CC} \rightarrow \mathrm{TT}$ mutations that can induce tumors (Pinnell, 2003). These are called "signature" due to their exclusivity to p53 gene mutations in UV-induced human skin cancers and are not present in either non-UVinduced human internal cancers or in mouse skin cancers induced by chemical carcinogens (Soehnge, Ouhtit et al. 
1997). Base substitutions (transversions), frameshift mutations, gene duplications, insertions and deletions can also be induced by UV light.

The various photoproducts that can be formed by UVB include cyclobutane pyrimidine dimers (CPDs) (e.g. thymine dimers), 6-4 photoproducts, cytosine photohydrates, crosslinks of DNA to DNA or protein and DNA strand breaks (Bowden, 2004). Thymine dimers however, occur most predominantly ( 75\%), are the most studied, are considered the main factors leading to cytotoxicity and are hotspots for mutations (Friedberg, 2001). The action spectra of these dimers fall maximally within the UVB range, specifically at around $300 \mathrm{~nm}$, although UVA can also induce thymine dimer formation (Pinnell, 2003). The latent period between the peak of its formation and prior UVB exposure is only about 1 hour, which implies that the cancer initiation phase occurs rapidly in contrast to the subsequent promotion and advancement phases. CPDs are formed when two adjacent pyrimidine residues form carbon-carbon links between their respective C-4 and C-5 atoms, thus creating a cyclobutane ring between the bases (Figure 6a). This results in the loss of UV absorption of thymine, although the reaction is reversible upon irradiation with short wavelength light at $240 \mathrm{~nm}$. During reversion, dimers return to their monomeric forms because of the differences in the absorption spectra between thymine and the thymine dimers (Sugiki et al. 1996).

The remainder DNA lesion type upon absorption of UVB light is mainly comprised of (6-4)-photoproducts (Figure 6b). These lesions on the other hand, create significant disturbances in the structure of the double helix when the C-6 and C-4 positions of adjacent pyrimidines bond. Both CPDs and (6-4)-photoproducts are highly mutagenic but CPDs are considered the major contributors to mutations, while (6-4)-photoproducts are more easily repaired in mammals in as early as 3 hours post-irradiation (Mitchell and Nairn, 1989). 


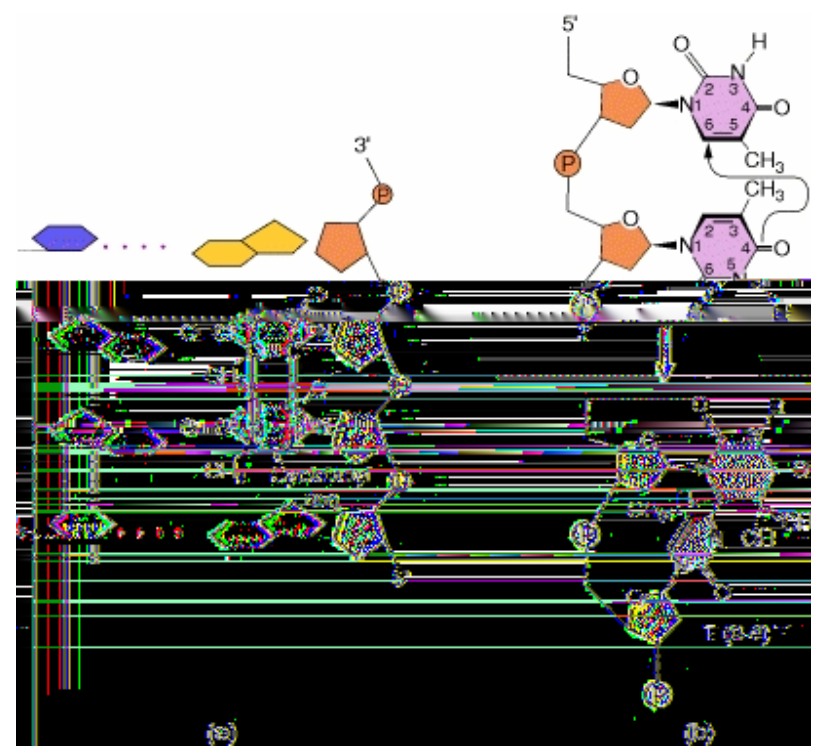

Figure 6. Structure of (a) a cyclobutane pyrimidine dimer and (b) 6-4 photo-product. Source: Griffiths et al. (1996).

These photoproduct lesions interfere with base pairing and thus, block replication as well as transcription. Thymine dimers present in promoter sequences inhibit transcription factor binding (Tommasi et al. 1996) and if present in the transcribed strand, prevents transcription elongation (Donahue et al. 1994). These disturbances in the transcription process could partly be accountable for their carcinogenic effects.

Other DNA lesions known to form after UV irradiation are pyrimidine monoadducts, purine dimers, adjacent A-T photoproduct and dimers of uracil, cytosine, uracil-thymine, cytosine-thymine, and uracil-cytosine. These represent lower than $1 \%$ of all DNA photodamage lesions and not much information is known about their mutagenicity (Kanjilal and Ananthaswamy, 1996).

The nucleotide base content of DNA has also been implicated in its sensitivity to UV irradiation. As adenine-thymine base pair increases within the strand, there is an observed increase in sensitivity to cell death by UV (Kaplan and Zavarine, 1962). This is perhaps a consequence of thymine dimer formation or because of the DNA's susceptibility to denaturation due to a decrease in overall G-C nucleotide content, which in turn doubles the rate of thymine dimer formation (Wacker et al. 1962).

Urocanic acid is mostly found in the upper layers of mammalian epidermis as a by-product from the breakdown of filaggrin, a protein that aggregates intermediate filaments and promotes the cross-linking of keratin (Anglin et al. 1961). Recent studies reveal that urocanic acid can be considered a second chromophore of significant biological importance (Simon, 2000). Intially, urocanic acid was believed to be a natural sunscreen because of its 
broad absorption spectrum for UV (Zenisck et al. 1955). However, it was determined that urocanic acid plays a role in UV-induced immunosuppression (De Fabo and Noonan, 1983) and that the highly reactive oxygen species (ROS) singlet oxygen is produced by the absorption of even a single photon of UV light by the trans-urocanic acid species which peaks at around $345 \mathrm{~nm}$. This free radical is capable of damaging cell membranes, which in turn produces more free radicals in the process.

\subsubsection{Reactive Oxygen Species}

Generation of free radicals such as ROS is a natural physiological occurrence during metabolism of tissues such as the skin (Darr and Fridovich, 1994). Oxygen molecules are mainly used during metabolism and energy production in the cell's mitochondria. A series of oxidation reactions involving subtraction of a single electron from oxygen produces superoxide anion, hydrogen peroxide, hydroxyl radical and lastly, water. Superoxide anion and hydroxyl radical are free radicals characterized by extreme reactivity to their immediate environment. Hydrogen peroxide, molecular oxygen and singlet oxygen are not free radicals but still reactive molecules that produce free radical species through oxidation (Pinnell, 2003). Collectively, these free radicals and reactive oxygen molecules are known as ROS.

Peroxides can form when fatty acids are exposed to UV radiation (Urbach, 1965). The basic structure of the cell membrane, the phospholipid bilayer, consists of unsaturated fatty acids such as linoleic and linolenic acid and the presence of lipid peroxides is believed to contribute to the instability of the membrane, most especially, lysosomal membranes (Desal et al. 1964). Reactive oxygen species are also known to alter genes and genetic processes (Tyrrell, 1996; Ahmed et al. 1999; Wenczl et al. 1997), DNA repair (Parsons and Hayward, 1985) and induce cytokines associated in the process of photoaging (Fisher et al. 1996; Fisher et al. 1997) and carcinogenesis (Runger, 1999).

Cells are protected from the damaging effects of these molecules by enzymes and controlled metabolic processes (Young et al. 2001), but the presence of oxidative stresses such as high metabolic demands, UV exposure, smoking and pollution may overwhelm the defense system and accumulation of the damage further contributes to the photoaging process. The enzymes catalase, glutathione reductase and glutathione peroxidase function to degrade 
hydrogen peroxide and lipid hydroperoxides, whereas superoxide dismutase degrade superoxide. Inherent antioxidants that provide additional protection include aqueous glutathione and ascorbic acid and lipid vitamin E and ubiquinol-10 found in membranes.

\subsubsection{DNA Damage}

Two of the main events involved in irradiation of a cell are damage and repair. The former is a factor of the dose and sensitivity of the target components, whereas the latter depends on the environment as well as the genetic and physiological state of the cell. The overall sensitivity of the cell, therefore, is a product of the balance between DNA damage and partial repair and absolute UV sensitivity can only be measured by deliberately removing the repair capacity.

Damage to DNA is considered to be the chief cause of a wide range of UV effects. Various chromosomal abnormalities have been found in UV treated cells. Studies on Chinese hamster cells by Ikushima \& Wolff (1974) and Trosko et al. (1965) showed that terminal deletions, DNA single strand breaks and chromatid exchanges increased after UV exposure at 265 or $280 \mathrm{~nm}$ in a dose-dependent manner. At $280 \mathrm{~nm}$, chromosomes also lose their smooth outlines, which can be observed even in severely damaged chromosomes. Other forms of DNA damage include protein-DNA crosslinks and thymine glycol.

The predominant effect of UVB radiation on DNA as previously mentioned however, is the formation of dimeric photoproducts between adjacent pyrimidine bases on one strand, most notable of which are cis-syn CPDs. In fact, approximately 100,000 CPD's per basal cell of the human epidermis form after three MEDs of 60-minute solar light exposure at noon in the summer in Kobe, Japan (Ichihashi et al. 1998). Among CPDs, thymine-cytosine (TC) and cytosine-cytosine (CC) dimers are considered the most mutagenic given that $\mathrm{TC} \rightarrow \mathrm{TT}$ and $\mathrm{CC} \rightarrow \mathrm{TT}$ are frequently seen in p53 gene mutations of cancer cells induced by UV irradiation (Daya-Grosjean et al. 1995). TT dimers in contrast, have low mutagenicity due to preferential incorporation of A residue to most non-specific lesions and consequent restoration of the A-T sequence.

Reactive oxygen species are also major contributors to DNA damage. Guanine oxidation occurs when DNA is exposed to either UVC or UVB even in the absence of a chromophore. This arises from the generation of 
singlet oxygen due to electron transfer from purine and pyrimidine bases in their triplet state (Ito and Kawanishi, 1997). In subsequent reactions guanine (G) residues are converted to 8-hydroxyguanine (8-OHdG), which pairs with adenine (A) instead of cytosine (C) during replication (Kielbassa et al, 1997). As such, 8-OHdG is considered as a suitable marker for oxidative stress.

\subsection{Skin Carcinogenesis}

Chronic exposure to UV radiation starting from childhood has been shown to induce most skin cancers (Ichihashi et al. 2003). The intricate processes involved in carcinogenesis can be summarized in three main stages namely, initiation, promotion and progression (Kanjilal and Ananthaswamy, 1996). The first step and primary requirement in carcinogenesis resulting from UVB exposure is the induction of DNA damage through the formation of cyclobutane pyrimidine dimers and pyrimidine [6-4] pyrimidone photoproducts (Mitchell, Greinert et al. 1999). Concurrently, ROS are generated eliciting further damage to DNA and other susceptible molecules (Pourzand and Tyrell, 1999). Also, previously existing mutations may remain dormant in the DNA until exposure to initiating agents such as UV radiation occurs. Upregulation of gene expression through different signal transduction pathways may play a role in the initiation of tumorigenesis. The damage sets off the rapid production of p53 that in turn triggers an increase in $\mathrm{p} 21$ production. The cell cycle is kept from proceeding and the cell shuts off DNA synthesis and cell replication to allow more time for DNA repair or to allow apoptosis of cells with severe DNA damage (Zambetti and Levine, 1993). Irreparable DNA damage and inability of the cells to undergo cell death however, often result in incorporation of the wrong bases into the DNA during synthesis. Hence, this increases the frequency of mutations, which depending on their type, may lead to loss of or inappropriate protein expression or generation of defective proteins. In many genes, multiple mutations at different loci are required to induce carcinogenesis and it takes, depending on the cell's lifespan, about three to seven mutational events on average to transform normal cells into cancer cells (Kanjilal and Ananthaswamy, 1996). Further UVB exposure and the additive effect of mutations in genes then induce the development of benign and eventually, malignant skin tumors where abnormal proliferation

involves imbalances in oxidation-antioxidation reactions, promotion of mitogenic and anti-apoptotic pathways, impaired cell cycle and apoptotic controls and sometimes reduced differentiation (Bowden, 2004). 
If signature UV photodamage mutations occur in the p53 gene (TP53) and nucleotide excision repair fails to eliminate them, the quality of the replicated genome suffers. In fact, the most frequently mutated gene identified in human cancers is the p53 gene with over 5000 mutations (Greenblatt, Bennett et al. 1994). There are currently 9 identified hotspot mutations in p53 that result in amino acid changes, which render the p53 protein non-functional (Ziegler, Leffell et al. 1993). Hotspot tandem mutations are readily detected at codons 245 and 247/248 in UVexposed normal human skin cell cultures and normal human skin biopsies (Nakazawa, English et al. 1994). Proliferation of keratinocytes with erroneous genome is a high risk factor at inducing actinic keratosis (Ziegler, Jonason et al. 1994). Actinic keratosis is a premalignant lesion that has a high frequency of p53 mutational incidents. In a case study conducted in Australia, normal skin biopsies with mutations at codons 247/248 of the p53 gene have a high risk of developing BCC (Ouhtit, in press). Around $>90 \%$ of skin SCCs and $50 \%$ of BCCs possess mutations in TP53 (Kraemer, 1997). These findings implicate the selective growth advantage introduced to cells by these mutations.

In contrast to other cancers such as colon cancer where p53 mutations occur late in the carcinoma stages, p53 gene mutations arise early in skin carcinogenesis (Jonason, Kunala et al. 1996). In mouse skin models, p53 mutations develop as early as the $4^{\text {th }}$ week of UV irradiation, which progressively increases to $50 \%$ at the $12^{\text {th }}$ week of chronic exposure (Ananthaswamy et al. 1997). The first sign of a mouse developing a tumor was at around the same period of maximum mutation frequency of 16 weeks. By 25 weeks, half the mice population had skin cancer. Such data strongly attests to the usefulness of detecting p53 mutations for early detection of skin cancer for the design of preventive programs.

If tumor development involves the accumulation of mutations in critical genes, epigenetic processes are known to be involved in tumor progression from pre-malignant to fully malignant; mitogenesis is initiated by tumor promoters such as growth factors and greatly enhances tumor progression; immune response is another critical mechanism that regulates elimination of pre-malignant cells; and angiogenesis or the formation of new blood vessels is necessary for the outgrowth of tumors. 


\subsubsection{Tumor Suppressor and Oncogenes}

Mutations that are critical to cancer development and hence induce transformations are those usually found in tumor suppressor genes, oncogenes or other cell proliferation regulatory genes (Soehnge, Ouhtit et al. 1997). Tumor suppressor genes serve as negative regulators of cell proliferation and usually need to be inactivated in both alleles (recessive inactivation) before totally losing its ability to restrict cell growth. Oncogenes, on the other hand, need only a single copy of the gene to be activated (dominant activation) to induce uncontrolled proliferation. Previously unmutated versions of oncogenes are termed protooncogenes. They normally facilitate the regulation of cell proliferation and differentiation and are classified into three major groups, which include: growth factor and growth factor receptors, signal transduction proteins and nuclear factors (Kanjilal and Ananthaswamy, 1996). Gatekeeper genes control cell proliferation and apoptosis including $R b, A P C$ and $p 53$. Caretaker genes are responsible for the integrity of the genome and include $X P, M M R, C S, A T M, B R C A-1$ and BRCA-2 (Levitt and Hickson, 2002). A necessary condition for carcinogenesis to develop is either through the overexpression of normal genes or normal expression of mutated gene products. Incomplete or incorrect repair of DNA lesions result in mutations of oncogenes such as ras and tumor suppressors such as p53 and patched. Although these occur rarely, such events lead to transformation and immortalization of cells, hence inducing tumorigenesis. Ample literature is available on specific genes of interest for skin cancer including the $p 53$ and patched tumor suppressor gene and the ras oncogene.

\subsubsection{Types of Skin Cancer}

Sunlight induced tumorigenesis are usually of epidermal origin. The two main types of skin cancer are melanoma and non-melanoma. Non-melanoma cancers primarily include basal cell (BCC) and squamous cell carcinoma (SCC). According to the American Cancer Society, about 1 million cases of basal and squamous cell carcinoma and 68,800 new cases of melanoma have been reported in 2009. Both SCC and BCC arise from epidermal keratinocytes but it is evident that the molecular pathogenesis behind their development are considerably different (Nickoloff, Qin et al. 2002). However, mutation of p53 occurs in around 90\% of SCC and BCC patients. 
UV exposure is predominantly associated with non-melanoma skin cancers although increasing evidence point towards the role of UV irradiation in influencing malignant melanoma development (Gloster and Brodland, 1996). Benign tumors (e.g. actinic keratosis) can also arise from basal layer keratinocyte or melanocyte proliferation. Patients with actinic keratoses, BCC and SCC often begin their overexposure to sunlight in childhood.

BCC's are initially shiny, dome-shaped structures surrounded by superficial blood vessels known as telangiectases (Figure 7). As the name implies, they often develop from basal cells often in the face around the eye and nose regions and currently comprise about $90 \%$ of all skin cancer cases in the U.S. These cancers are common in people with weakened immune system and rarely metastasize, but are known to infiltrate surrounding tissue. Several connective tissue changes such as collagen and elastic tissue reduction, argyophilic fiber proliferation, acid mucopolysaccharide, mast cells and fibroblast increase and sometimes formation of new elastic tissue are correlated with the development of basal cell epitheliomas (Dobson, 1963; Zackheim, 1963; Mehregan et al. 1964). "Signature" UV photodamage mutations that occur in patched genes or other effectors in the hedgehog pathway signaling are associated with the development of a basal cell carcinoma (Bale and $\mathrm{Yu}, 2001$ ).
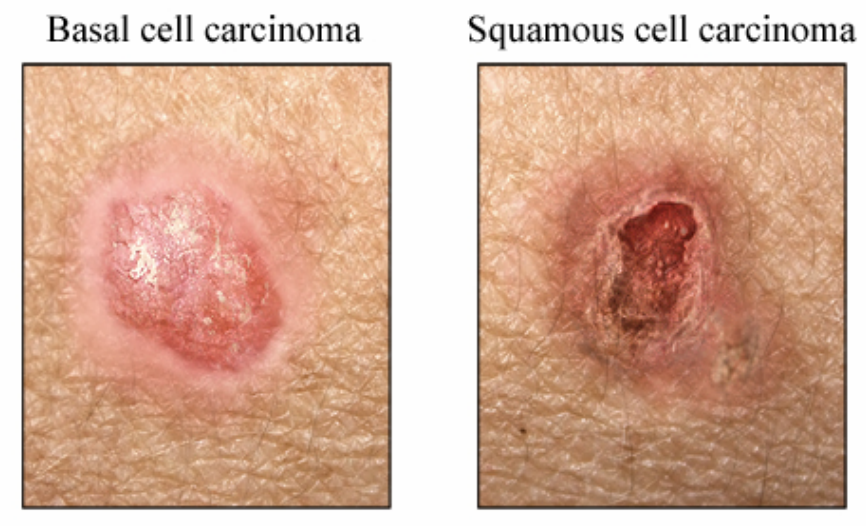

- Healthwise, Incorporated

Figure 7. Basal and squamous cell carcinoma. Source: Healthwise, Incorporated, (2008).

A more aggressive and invasive non-melanoma cancer is squamous cell carcinoma. This arises from keratinocyts and begins mainly on the back of the hands, on the ears, forehead or lower lips and is characterized by a firm red bump, scaly, bleeding or crusty growth or a sore that does not heal. SCC's may grow rapidly and spread to neighboring lymph nodes. UVB is attributed to most cases of SCC development but a peak in the UV action spectrum occurs around the UVA wavelengths of 320-400 nm as well (de Gruijl et al. 1994). In several chronic UV exposure studies (Mowry, 1958; Hale et al. 1960) spanning 4-6 week periods, hyperplasia in the stratum corneum, 
granulosum, spinosum and basement membrane was initially observed. Further exposures induced the development of squamous cell carcinoma while developing clumping of the nuclear chromatin, increased mitoses, loss of polarity and differences in size and shape. At the stage at which the malignancy started to invade aggressively, the skin tissue loses its basement membrane and connective tissue changes become difficult to assess. A remarkable structural change across the stages of cancer progression is that of the basement membrane. At first, it exhibits hyperplasia without much change in its morphology. Eventually, the membrane becomes thicker, clumped and frayed and ultimately disappears with invasive malignancy. At the cellular level, there is a marked acceleration of cell division and DNA synthesis observed from benign to malignant stage of squamous cell carcinoma, while mutation in both alleles of the p53 gene is often observed to give rise to squamous cell carcinoma.

Melanomas are the most studied type of skin cancer and are also the most lethal. They begin in melanocytes and in contrast to majority of cancers which target the p53 pathway and induce mutations on the p53 gene itself, melanomas are characterized by high levels of wild-type p53 and consequent upregulation of p21 proteins. Paradoxically, they exhibit high resistance to cell death despite aggressive treatments that are otherwise highly effective against cancer cells possessing normal p53 functionality. It has been established that malignant melanoma is mainly a genetic disease but recent evidence on the unraveling of the melanoma genome has shown that most of the 33,000 mutations found bear the imprint of mutations due to UV exposure. Around $65 \%-90 \%$ of melanomas arise due to UV irradiation (Armstrong and Kricker, 1993). A crucial indication of its progression to malignant form is if a mole changes in shape, outline or color or starts to bleed. As evidenced by the malignant nature of melanoma, the tumor-suppressor function of this wild-type p53 is clearly defective. This lack of correlation however, may possibly be accounted for by a still unknown factor that may induce variations in posttranslational modifications of the p53 protein or induce defects in protein cofactors involved in critical p53 functions (Kichina et al. 2003). Indeed, it had been shown that a mutation in a protein (chk2/hcds1) involved in signaling the presence of DNA damage to the p53 protein is accountable for inactivation of p53 in Li-Fraumeni Syndrome patients (Satyamoorthy et al. 2000). 


\subsection{UV Protective and Repair Mechanisms}

\subsubsection{Protective Barrier}

Strangely enough, healthy formation of the stratum corneum entailing the development of dead keratinocytes in the superficial layer of the epidermis is necessary to maintain cutaneous homeostasis. The stratum corneum is only about one-tenth the thickness of the rest of the epidermis yet it has double the number of cells packed in such a highly optically dense layer of about 15 cells stacked on top of each other. For this reason, they are very efficient at providing a barrier, which is the single most important function of skin (Nickoloff, Qin et al. 2002). Moreover, the stratum corneum alone is loaded with a high concentration of proteins (75-80\%), which aid in creating a considerable resistance to the passage of water, topical chemicals and light to a certain extent (Nemes and Steinert, 1999). The stratum corneum scatters incident light by numerous reflection and refraction at skin discontinuities. The Malpighian layer of the epidermis, which is basically its viable region has intracellular components populated by protein, lipids, monofilaments, keratohyaline granules and melanosomes that tend to dissipate UV radiation through efficient scattering of short wavelength radiation $(<320 \mathrm{~nm})$ and thus, prevent deep penetration of UV into the body. Likewise, cells in the stratum germinativum are fully hydrated and their intact cell and nuclear membranes along with the keratohyaline granules of the stratum granulosum have optically discontinuous surfaces.

Furthermore, the absorption spectrum of ultraviolet light in skin is considerably modified from its normal action spectrum due to the fact that the superficial layers serve as spectral filters for the underlying layers. Aside

from attenuating effects of the superficial layers, certain wavelengths are absorbed more effectively in certain regions, thereby altering the wavelength composition as incident UV light goes deeper into the tissue. Keratins, which are abundantly produced in the stratum corneum are responsible for considerable absorption between the 260 and $290 \mathrm{~nm}$ wavelengths. Other components that absorb radiation between 260-290 $\mathrm{nm}$ include histidine, urocanic acid, small peptides, cholesterol and phospholipids of the stratum corneum and the nucleic acids of the nucleated cells in the Malphigian layer. 
However, more critical components in shielding the skin from the effects of UV irradiation are the melanin granules predominantly found in the basal layer and stratum corneum. Apart from extensive absorption of damaging UV, infrared and visible light radiation, they are also involved in considerable scattering of light and dissipation of absorbed energy as heat. Melanin granules, by accumulating in the perinuclear region, provide a protective shield by preventing the nuclei from being hit directly by radiation.

\subsubsection{Antioxidants}

The skin has intrinsic stores of antioxidants that serve to protect itself from photodamage and methods to augment this process by topical application of antioxidants have been proven to further diminish the harmful effects of free radicals (Pinnell, 2003). Antioxidants can act either intra- or extracellularly and intracellular antioxidants are often enzymes. Catalase is found especially in peroxisomes at relatively higher concentrations in the epidermis than in the dermis and serves to detoxify hydrogen peroxide, while glutathione peroxidase and glutathione reductase function to reduce hydrogen peroxide and lipid hydroperoxides using glutathione. Copper-zinc and manganese superoxide dismutase protect against superoxide in cells whereas extracellular superoxide dismutase act extracellularly. Non-enzyme antioxidants include aqueous L-ascorbic acid (Vitamin C), glutathione in the cytoplasm, vitamin E located in membranes and ubiquinol found in mitochondria. However, these often prove inadequate due to repeated exposure to sunlight and other sources of free-radicals (e.g. smoking, pollution). Catalase and superoxide dismutase activity in particular, are considerably diminished upon UVA exposure of skin fibroblasts (Shindo et al. 1997) and ubiquinol and glutathione were observed to be readily destroyed upon irradiation of murine skin (Shindo et al. 1993). Decreased antioxidant levels of ascorbic acid, $\alpha$-tocopherol and glutathione are also correlated with actinic keratosis and basal cell carcinoma (Vural et al. 1999).

L-ascorbic acid is highly soluble and is considered the body's major aqueous reducing agent (Rumsey et al. 1999) with many known functions. It reacts with harmful free radicals by donating an electron, thereby generating a relatively stable ascorbate free radical that easily pairs with nearby free radicals. Loss of the second electron produces dehydroascorbic acid, which can be regenerated by dehydroascorbic acid reductase or more often degrades upon opening of the lactone ring. It is also known to be involved in collagen synthesis by being a cofactor for prolyl 
and lysyl hydroxylase enzymes required for molecular stability and intermolecular cross-linking (Kivirikko and Myllyla, 1985), as well as in the regulation of transcription of genes for collagen synthesis (Tajima and Pinnell, 1996). L-ascorbic acid also decreases photoaging by inhibiting increased elastin biosynthesis (Davidson, 1997) and reducing abnormal pigmentation in melanocyte cells by decreasing tyrosinase levels (Maeda and Fukuda, 1996). Lastly, it had been reported to aid in keratinocyte differentiation (Pasonen-Seppanen et al. 2001; Savini et al. 2002) and in inducing sphingolipid synthesis (Ponec et al. 1997; Uchida et al. 2001), thus enhancing the epidermal barrier function.

Due to a loss of function mutation in L-gulono- $\chi$-lactone oxidase, the human species are the only eukaryotic organisms that cannot synthesize L-ascorbic acid (Nishikimi et al. 1994) and must acquire it through the diet. But with high amounts of oral supplementation, intrinsic control mechanisms constrain its absorption up to a certain threshold (Levine et al. 2001). Alternatively, topical application with removal of the extra ionic charge on the molecule proves to be a good method to enhance its levels in skin (Pinnell et al. 2001). Studies have shown that L-ascorbic acid protects porcine skin from erythema and sunburn (Darr et al. 1992) and mice from UVB immunosuppression (Nakamura et al. 1997), and enhances procollagen I and III transcription and processing enzymes in human skin (Nusgens et al. 2001)

Vitamin E is the body's major lipid antioxidant (Packer et al. 2001) and can exists in either tocopherol or tocotrienol form. In particular, $\alpha$-tocopherol is the predominant form consumed since it is easily transferred into lipoproteins (Azzi et al. 2000). Vitamin E is found at high concentrations in the deeper layers of the stratum corneum where they are distributed by the skin's oil secretions (Thiele, 2001) to protect lipid structures as well as proteins from oxidation. The antioxidant functions mainly to inhibit lipid peroxidation, i.e. a chain reaction of peroxyl radical formation which occurs when the ROS reacts with membrane lipids resulting in the weakening of membrane structure integrity (Munne-Bosch and Alegre, 2002). This chain reaction can be terminated by a reaction between Vitamin E and the peroxyl radical. Moreover, Vitamin E can scavenge singlet oxygen and be regenerated to its reduced form by L-ascorbic acid (Packer et al. 2001). Animal studies revealed numerous important findings on its photoprotective properties including protection against erythema (Roshchupkin et al. 1979), lipid peroxidation (Lopez-Torres et al. 1998), UV-induced photoaging (Jurkiewicz et al. 1995), UV immunosuppression (Yuen and Halliday, 1997), melanogenesis (Ichihashi et al. 1999) and photocarcinogenesis (Gensler and Magdaleno, 1991). 
The cancer prevention mechanism was further revealed to be due to inhibition of cyclopyrimidine dimer formation in the epidermal p53 gene in mice (Chen et al. 1997).

Currently available topical antioxidants provide additional protection by supplementing the diminished stores of natural antioxidants consumed in the body during the series of redox processes involved in breaking down ROS. Problem areas needing special protection can be especially targeted with direct application. Topical application also eliminates the complexities associated with dietary and oral supplementation such as systemic distribution, absorption and solubility, which limits the total concentration of antioxidants reaching the skin. There are however, disadvantages with using topical antioxidants including the characteristic instability of antioxidants that is essential to their roles as redox reactants, their deep coloration and the need for high concentrations. For instance, glutathione is a tripeptide with ionic charges which impedes with its ready absorption into the skin. Hence, formulation of antioxidants for cosmetic purposes continues to be a challenge to many skin care specialists.

\subsection{3. p53-mediated Cell Cycle Arrest}

An disturbance in cell division is observed after UV irradiation and follows a dose dependent trend, with large doses ultimately rendering sterility to the cell (Urbach, 1969). In the latter case, a cell undergoes growth arrest for durations of hours, days or weeks, followed by cell shrinkage and eventually, death. Yet there are cases where cells survive and proceed with the normal process of growth and division observed in controls, as well as cells that die even after an apparently normal division as an effect of lethal mutations.

Focused irradiation of specific organelles at different stages of mitosis reveals their different roles in cell division. An important discovery was that irradiation of the nucleus more often results in cell cycle arrest than irradiation of the cytoplasm. This is an effect of damage to chromosomes and errors in their partitioning during subsequent mitosis, thus resulting in segregation of whole chromosomes. Irradiation of spindle fiber attachment during mitosis known as the kinetochore, causes random movement of the chromosomes during partition. Specifically targeting the individual chromosomes in heart-cell tissue cultures triggers a loss of characteristic UV absorption in nucleic acids attributed to the loss of DNA in such regions (Bloom, 1965). UV microbeam irradiation of centrioles halts cell division perhaps due to a hindrance in the cleavage step (Rustad, 1960). 
It is believed that exposure to UVB induces p53 in response to excised thymine dimers (Goukassian, Eller et al. 1999). p53 accumulation after DNA damage by UVB is critical in initiating DNA repair (genome protection) or apoptosis of cells with damaged DNA (tissue protection). Several cell cycle regulators are expressed upon the accumulation of p53 (Figure 8). IGF-BP3, Gadd45 and D1 or G cyclin are known to induce cell cycle arrest although their mechanisms of action have yet to be elucidated. The most notable and main immediate effector of p53 is p21 (also known as Cip1, Sdi1, CDKI, MDA-6, CDKN1 or Pic1, encoded by the Wafl gene), which inhibits cyclin-dependent kinases. It is known as the universal cell cycle inhibitor, which inhibits Cdk/Cyclin complex formation and hence, impede DNA replication and cell cycle progression (Harper et al. 1993). Cell cycle arrest may occur in at least two checkpoints: before DNA replication (G1/S phase) or before chromosome segregation (G2/M phase) (Herzinger et al. 1995). Cell cycle is directly controlled by cyclin-dependent kinases by hyperphosphorylating and inactivating cell cycle inhibitors such as Rb protein and p107 and p130 (Soehnge, Ouhtit et al. 1997). But accumulation of p53 after DNA damage arrests the cell cycle at the G1 checkpoint before S phase, which is crucial in repairing the damage before DNA is replicated (Huang, Clarkin et al. 1996). By binding to proliferating cell nuclear antigen (PCNA), p21 allows DNA repair to proceed but not DNA replication (FloresRozas et al. 1994; Waga et al. 1994; Podust et al. 1995), hence inhibiting the expression of growth responsive genes and DNA replication (Fotedar, Bendjennat et al. 2004). The resulting E Cyclin/PCNA/Waf1 complex then leads to the accumulation of hypophosphorylated $\mathrm{pRb}$. This in turn, releases E2F which is required for initiating DNA synthesis in the S-phase of the cell cycle (Harper, Adami et al. 1993). 


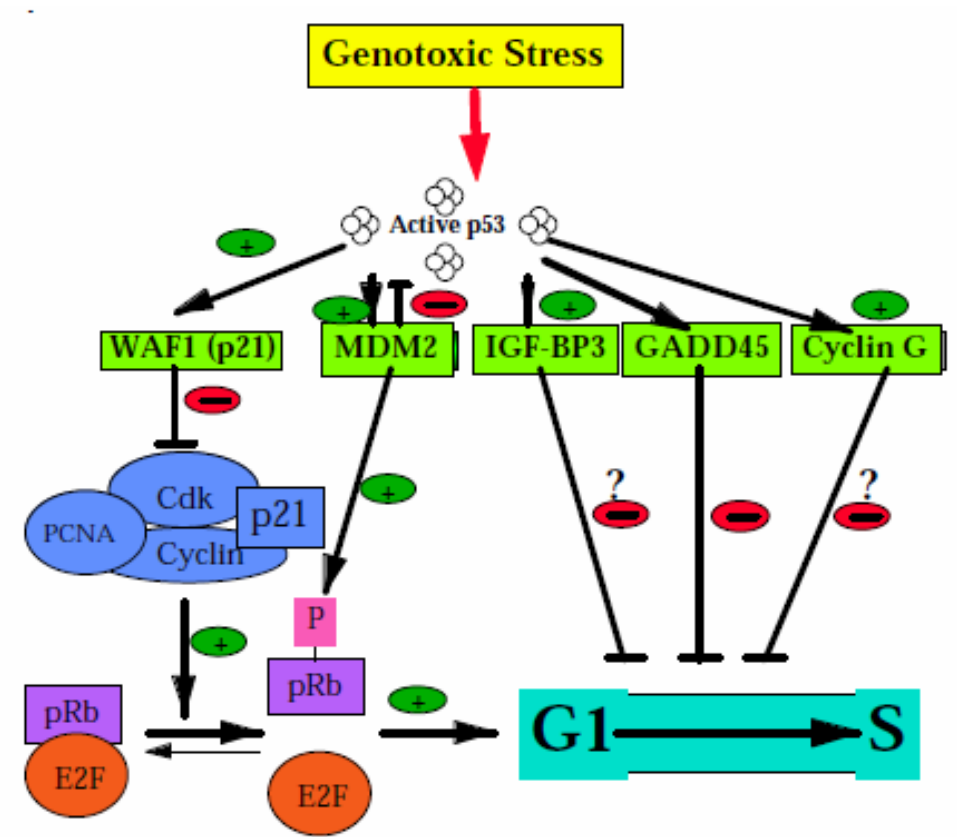

Figure 8. p53 accumulation and cell cycle proteins. Source: Soehnge and Ouhtit, (1997).

\subsubsection{DNA Repair}

DNA repair is deemed to be the chief biological response to DNA damage (Singh and Agarwal, 2005). The highly efficient anti-radiation repair system may have evolved as a testimony to the important relationship between UV light, living cells and visible light. Cells maintain a natural balance between the level of injury and the effectiveness of DNA repair. In cases skewed towards severe injuries or inefficient DNA repair, the frequency of mutation rises, resulting in a high risk of cancer development if cells do not undergo apoptosis (Kunkel 2004). DNA repair process is critical in skin cancer prevention as evidenced by Xeroderma pigmentosum (XP) patients whose DNA repair processes (i.e. NER) are defective. XP is a genetic disorder that renders the patients extremely susceptible to skin carcinogenesis characterized by the general failure to repair the thymine dimers in the DNA (Cleaver and Kraemer, 1989). Two of the most important mechanisms of DNA repair are nucleotide excision repair (NER) and postreplication repair by DNA polymerases, the latter of which involves synthesis of DNA in an errorfree process. Moreover, base excision repair (BER) system is capable of repairing 8-OHdG lesions and other ROS, hydrolysis and methylating agent-induced DNA damage. 
NER is a highly-conserved process that can induce cell growth inhibition and completely repair DNA damage before replication in the absence of light (Mitchell et al. 1985; Wood, 1997). It has been shown to repair both CPDs and (6-4) photoproducts. NER however, is not specific to UV irradiation but is likewise involved in the repair of damage due to ionizing radiation and DNA damaging chemicals (e.g. antibiotics and nitrous acid). This repair process can be initiated from two independent subpathways depending on the function of the damaged DNA: the global genomic repair (GGR) and transcription-coupled repair (TCR). Transcription-coupled repair was discovered upon the observation that CPDs in genes that are actively transcribed have a high probability of being repaired as opposed to non-transcribing regions in the genome in hamster and human cells (Bohr et al. 1985; Vreeswijk et al. 1994). CPD lesions are only repaired efficiently by the TCR subpathway (Mitchell and Nairn, 1989; Hanawalt, 1991). GGR is peculiar since it is highly influenced by p53 activation or loss of function but only marginally by p21 upregulation (Smith, Ford et al. 2000).

In general however, the overall NER process and subsequent steps can be summarized in five main steps (Aboussekhra et al. 1995): first, the cell recognizes a DNA lesion and unwinds the DNA double helix locally surrounding the lesion; incisions on a single strand are then made on both sides flanking the damaged region; next, the damage on the single strand is excised for up to 32 bases; DNA synthesis is then initiated to replace the lesion that was removed and fill in the gaps; and lastly, both ends of the newly synthesized DNA are ligated to the original DNA strand (Figure 9). This repair mechanism has also been identified to constantly act on dimer photoproducts (Goukassian et al. 2000). In cases when repair is incomplete, $\mathrm{C} \rightarrow \mathrm{T}$ and $\mathrm{CC} \rightarrow \mathrm{TT}$ mutations, which are distinctive to UV photodamage, may linger in the DNA strands. In such cases that the extent of damage to the genome is significant, p53 and its downstream effectors induce apoptosis of the irradiated keratinocytes (Goukassian, Eller $e t$ al. 1999). 


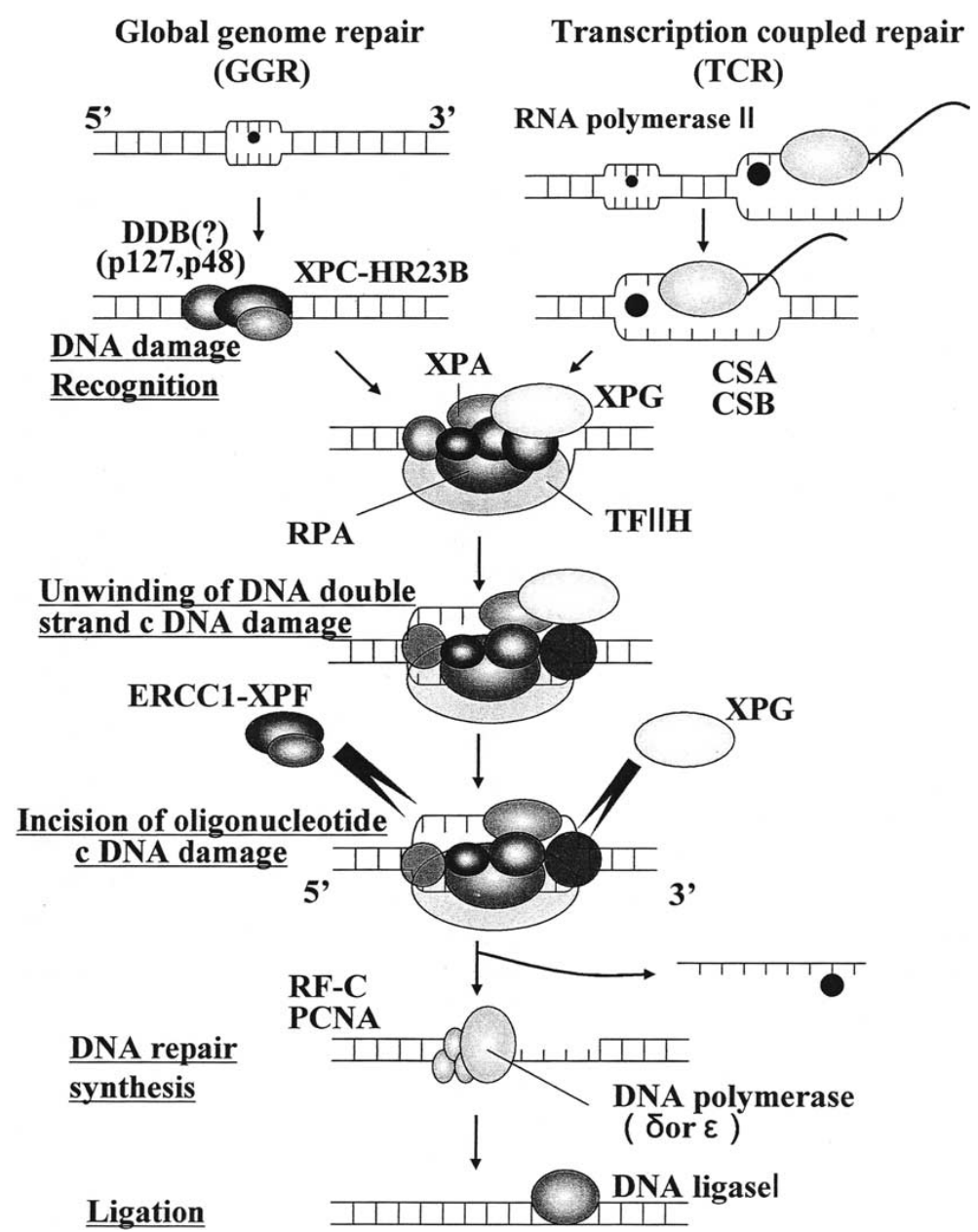

Figure 9. Chronological steps involved in nucleotide excision repair. Source: Ichihashi et al. (2003).

\subsubsection{Apoptosis}

In case of severe damage, the cell undergoes apoptosis or programmed cell death, which is a genetically encoded, controlled type of cell death distinct from necrosis. In necrosis, injury to the cell results in explosion and release of its contents, thereby inciting the inflammatory response. In apoptosis, the cell autodigests itself and is removed by roaming macrophages. In recent years, oncologists have focused on apoptosis as one of the main pathways to defeat cancer (Evan and Vousden, 2001). One of the most critical issues to investigate in cancer protection is to pinpoint which, if any, pro-survival and pro-cell death factors are exploited or inhibted by malignant cells. 
The two pathways employed by cells undergoing apoptosis are the extrinsic and intrinsic pathways. As the name implies, the extrinsic pathway involves the cell ultimately succumbing to pro-apoptotic signals received from outside the cell. The intrinsic pathway on the other hand, involves internal signaling triggered by considerable damage to DNA due to stressors such as chemicals, heat and radiation. For cancer to progress, both pathways should be disrupted. Either way, the central executioners in this final phase of apoptosis are a group of enzymes released into the cytoplasm to auto-digest proteins known as caspases (cysteine-aspartic acid proteases).

In keratinocytes, the response to UV activates various death pathways within the cell: death receptors (e.g. Fas and other TNF family) are known to cluster in the plasma membrane upon irradiation (Leverkus, Yaar et al. 1997); reactive oxygen species caused by photodamage triggers the release of pro-apoptotic effector molecules such as cytochrome C and Apaf-1 (Denning, Wang et al. 1998); and DNA damage causes the induction of p53 (Tron, Trotter et al. 1998) and MAP kinase families (Assefa, Garmyn et al. 1997). These pathways serve as redundant safety systems that ensure death to keratinocytes in the presence of an apoptic signal even if one pathway is compromised.

Cellular proofreading is an apoptotic response driven by p53 signaling wherein keratinocytes undergo cell death instead of damage repair (Brash, Ziegler et al. 1996) and may proceed in two independent pathways as seen in Figure 10. In the first pathway, p53 enhances transcription, thereby upregulating Bax, IGF-BP3 and Fas proteins, and downregulates the expression of Bcl2, IGF-1R and IGFII to induce apoptosis (Harris, 1996). The second pathway relies on 553 protein-protein interaction by binding of p53 protein to DNA synthesis proteins such as replicating protein antigen (RPA) (Dutta et al. 1993) and DNA repair proteins such as TFIIH, XPB and XPD DNA helicase, p62 and topoisomerase I (Wang et al. 1995; Gobert et al. 1996). It is important to note that all p53 mutation hotspots results in the expression of mutant p53 protein that inhibit p53-dependent transcription pathway (first pathway) (Brachmann et al. 1996).

Expression of Bax protein has also been observed to suppress transformation (Zong, Lindsten et al. 2001). This is also believed to be the pathway by which certain human papillomavirus (HPV) cause skin cancer through inhibition of Bak-mediated apoptosis. Likewise, disturbances in Fas/FasL, has been instrumental in the apoptoticresistant phenotype in several types of cancer such as SCC and melanoma where the tumor cells lose the ability to express Fas death receptor (CD95) (Hill, Ouhtit et al. 1999). 


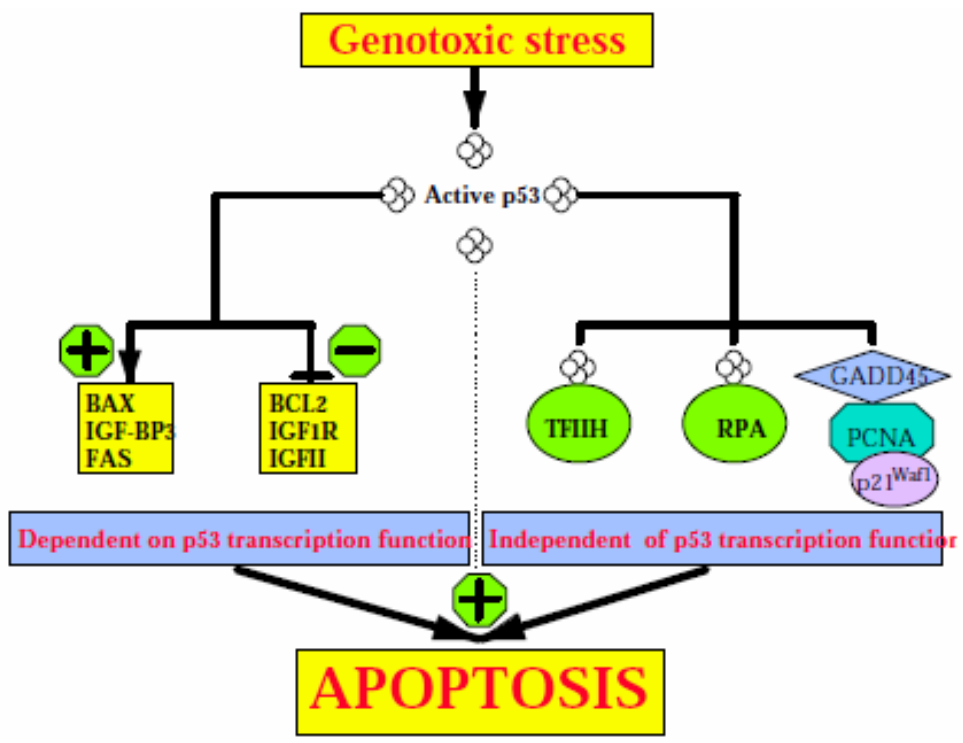

Figure 10. p53-dependent and independent apoptotic pathways. Source: Soehnge and Ouhtit, (1997).

The morphology exhibited by cells during this process is highly similar even in various cell types (Lockshin and Zakeri, 1990; Williams, 1991). As observed with an electron microscope, the nuclear chromatin initially aggregates into uniformly compact masses along the nuclear membrane as the cytoplasm decreases in size. Hoechst staining of apoptotic nuclei reveal bright fluorescence within the periphery of the nuclear membrane and absence of fluorescence at the middle of the nucleus due to perinuclear aggregation of chromatin and dissolution of the nucleolus (Oberhammer et al. 1993). Eventually, the nucleus breaks up into discrete fragments as the cell itself also degrades (Wyllie et al. 1980). Finally, cells ultimately lyse and lose their chromatin structure altogether after all of the DNA gets digested. The exact molecular mechanisms behind these events are still not clear but some studies were able to demonstrate that the entire nucleus becomes acidified during apoptosis (Gottlieb et al. 1995; Li and Eastman 1995; Perez-Sala et al. 1995). These point toward a possible role for lysosomes in releasing their digestive contents including acidic endonucleases after fusing with the nucleus, thus inducing DNA hydrolysis (Barry and Eastman, 1993). Alternatively, the lysosomes may lower the $\mathrm{pH}$ around the nucleus and hence, activate resident endonucleases within the nucleus. 


\subsection{Phospholipids: Structure and Function}

Phospholipids are monounsaturated fatty acids composed of a polar head group and two non-polar tails and are commonly present in membranes. The major membrane phospholipids in mammals include phosphatidylcholine, phosphatidylethanolamine and sphingomyelin. Sphingomyelins, in particular, are the dominant phospholipids that also belong to the sphingolipid class. They serve as major mammalian cell membrane phospholipids, reservoirs for ceramides and as constituent of nerves. Membrane phospholipids ensure the structural integrity of the membrane as well as influence the function of enzymes and transport proteins and receptors, which are essential in mediating several metabolic processes such as transport of nutrients to the cells and intercellular signaling. Phosphatidylserine is localized intracellularly and participates in organizing transport proteins and cell membrane enzymes, thereby facilitating cell-to-cell communication and signal transduction by conveying external signals to the inside of the cell. Sphingolipids are present in all eukaryotic cells and as of yet are thought to have at least three functions: structure, recognition and signal transduction (Merrill Jr, Sandhoff et al. 2002). Their signaling functions have been shown to confer protective effects against colon carcinogenesis. In general, they may possess curative properties on bowel inflammation (e.g. Krohn's disease). Sphingolipids are present in serum lipoproteins, especially LDL, and in lipidrich skin. They may aid in lowering cholesterol levels by competeng with their uptake and loading in intestinal micelles as well as raise the levels of HDL or "good" cholesterol. Gangliosides are yet another group of complex glycosphingolipids found in milk fat globule membrane that have been observed to induce pro-inflammatory mediators in the mucosas lining of the intestines after acute exposure to bacterial endotoxins.

Around $1 \%$ of total lipids in bovine milk are phospholipids, which is found in milk fat globule membranes, conferring structural stability to globules (Jensen et al. 1991). It is possible to obtain them in concentrated quantities through sophisticated milk processing technology. Milk phospholipids are known to contain high concentration of sphingolipids.

Sphingomyelins comprise the structural framework of plasma membranes (Figure 11) where they provide critical barrier to water loss, and can also be found in cytoplasmic vesicles (including Golgi bodies, endosomes and lysosomes) involved in responding to external agonists (Bell et al. 1993), as well as in the mitochondria and nucleus, though their exact locations in these organelles are still unknown (Merrill Jr, Sandhoff et al. 2002). A physical 
property that makes them suitable as membrane structures is their high phase transition temperature, which helps keep the membrane fluid at a reasonably wide temperature range. They can serve as receptors for antibodies, bacteria and viruses, as ligands for extracellular matrix proteins, receptors of neighboring cells and as second messengers for a variety of chemicals (e.g. TNF- $\alpha$, IL-1 $\beta$, nerve growth factor and $1 \alpha, 25$-dihydroxyvitamin $\mathrm{D}_{3}$ ) that trigger complex signaling pathways.

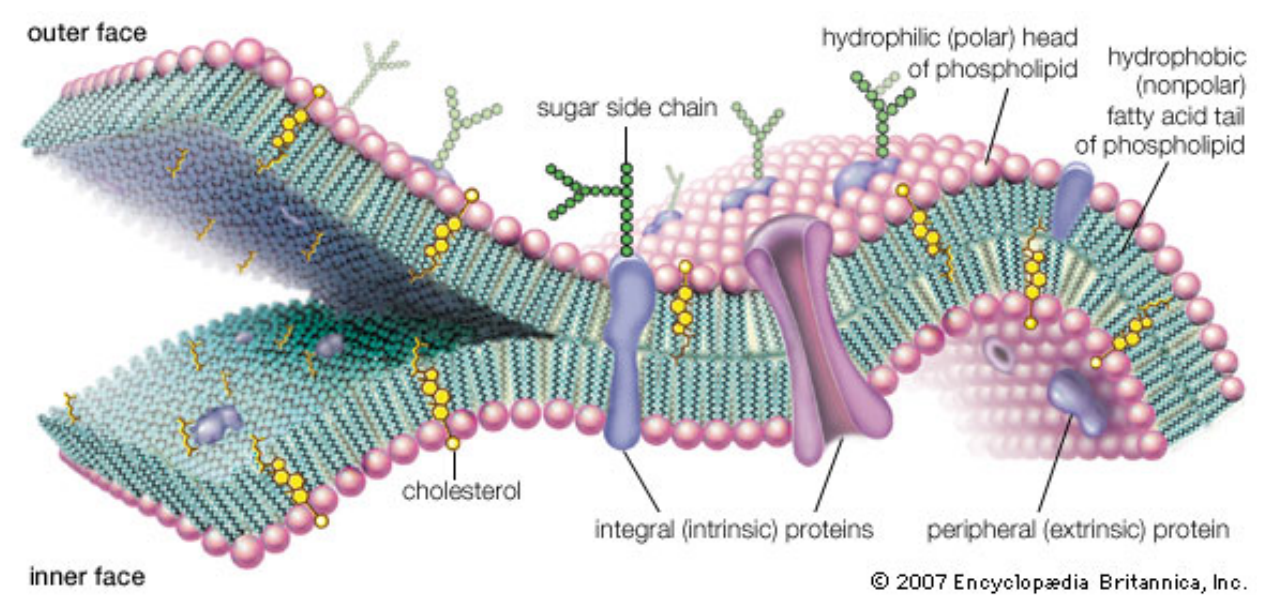

Figure 11. Phospholipid membrane bilayer components. Source: Britannica.com

During normal turnover and synthesis of sphingolipids in the cell, sphingomyelin structures are constantly being converted into intermediates by reversible break down processes collectively known as the sphingomyelin cycle (Figure 12). In addition, extracellular agents such as growth factors and cytokines activate the sphingomyelin degradation pathway in response to stresses including UV and $\gamma$-irradiation, serum deprivation, hypoxia, heat and chemotherapy (Schmelz, 2004). Catalyzed by different enzymes, each sphingomyelin molecule is cleaved into simpler compounds. Initially, headgroups are removed to form the first metabolite known as ceramide. This is then broken down to sphingosine before finally being activated by phosphorylation to sphingosine-1-phosphate (SPP). Although these metabolites do not belong within the phospholipid class per se but are rather recognized as sphingolipids, it is worth discussing their physiological effects since the metabolism of these compounds is linked to the constant breakdown and turnover of sphingomyelin. 


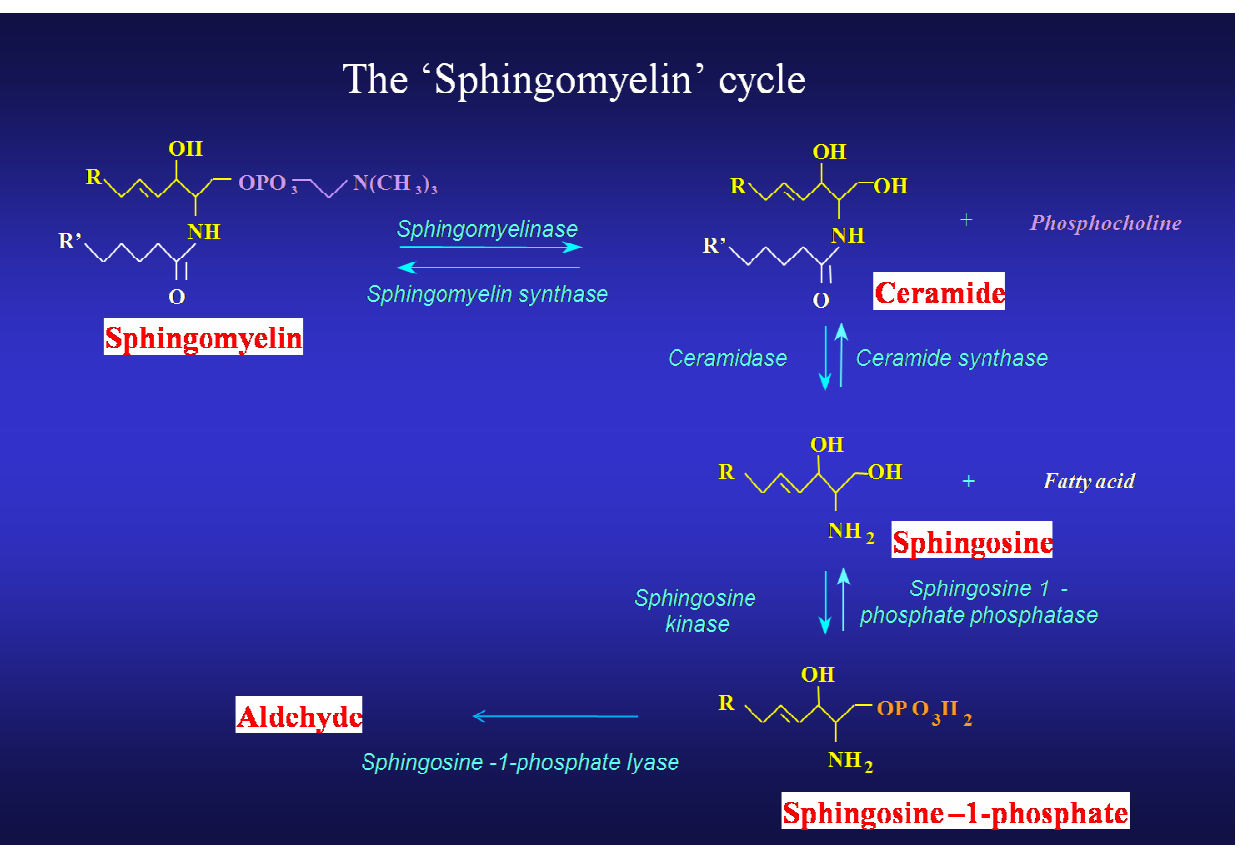

Figure 12. Sphingomyelin conversion cycle. Source: Kelly, (2009).

The field of sphingolipid signaling has only recently emerged and a few studies have given light on the action of its metabolites on multiple downstream targets that modulate cell behavior. Aside from providing a fluid structure to the cytoskeletal membrane, sphingolipids have been recognized to have diverse functions in regulating cell growth, transformation, intercellular communication, intracellular signal transduction and even cell death (Merrill, Schmelz et al. 1995). Their backbones in particular - sphingosine, ceramides and sphingosine-1-phosphate are currently believed to play some functions in cell regulatory processes involved in carcinogenesis. Ceramide and sphingosine trigger cell cycle arrest, differentiation or apoptosis in many transformed cell lines (Spiegel and Merrill, 1996; Mathias et al. 1998; Huwiler et al. 2000). On the contrary, sphingosine-1-phosphate stimulates cell growth, suppresses apoptosis, enhances cell survival, modulates adhesion and cell motility and induces cell differentiation (Spiegel and Milstein, 2003).

\subsection{Phospholipid Components in the Epidermis}

As basal cells migrate through successive epidermal layers, the stem cells likewise mature sequentially until they differentiate completely in the stratum corneum. For this reason, the lipid components at each layer 
change considerably during transport to the surface, where enzymes degrade phospholipids into glycerol and free fatty acids and glucosylceramides are degraded into ceramides. Hence, the major lipid components of the stratum corneum are cholesterol, free fatty acids and ceramides (Coderch, Lopez et al. 2003).

As described previously (see Skin structure layer section), the stratum corneum consists mainly of corneocytes and is surrounded by crystalline lamellar lipid regions. Intercellular bilayer can be found within intercellular spaces whereas neutral lipids enrich the membrane region of the stratum corneum (Coderch, Lopez et al. 2003). However, in contrast to other lipid bilayers in the body, phospholipids are absent and stratum corneum lipids consist mostly of ceramides, fatty acids, cholesterol, cholesterol sulfate and sterol/wax esters. Ceramides account for one of the major lipid components (40-50\%) of lamellar sheets in the intercellular spaces of the stratum corneum (Coderch, Lopez et al. 2003) and are generated from the hydrolysis of sphingomyelin and glucosylceramide in the stratum granulosum.. The presence of this complex group of sphingolipids offers stability, structure and impermeable barrier properties to the epidermis to combat dehydration and stress. Reduced levels of ceramides is often associated with atopic dermatitis and is currently considered as one of the major causes of barrier disruption and dry skin in this condition (Imokawa et al. 1991).

Lipid supplements formulated to be identical to skin's normal lipid composition and especially ceramide supplements can be used to treat a wide range of diseased skin conditions. In a study conducted by Man et al (1996) using murine and human skin models, topical administration of stratum coreneum lipid mixture including cholesterol, ceramide and free fatty acid resulted in their absorption into the nucleated layers of the epidermis, thereby repairing the induced damage to water barrier function. The finding also showed that application of ceramide, cholesterol or fatty acid alone, or a mixture of two of these induced barrier recovery over time. Hence, it was concluded that a balance in the ratio of the three lipids is necessary for barrier homeostasis and recovery. Alternatively, enhancement of natural lipid synthesis in the skin through topical administration of lipid precursors has also been shown to improve barrier function (Coderch, Lopez et al. 2003). 


\subsection{Systemic Fate of Dietary Lipids}

The backbones, fatty acids and headgroups that make up sphingolipids from different types of food are highly diverse. Sphingolipid species present in food sources from mammalian tissues such as beef, poultry and milk include ceramides, sphingomyelin, cerebrosides, gangliotides and sulfatides (Vesper, Schmelz et al. 1999). Sphingolipids are highly abundant in foods such as meats $(\sim 0.3-0.5 \mu \mathrm{mol} / \mathrm{g})$, dairy $(0.5-1 \mu \mathrm{mol} / \mathrm{g})$, salmon $(0.2$ $\mu \mathrm{mol} / \mathrm{g})$, chicken $(0.53 \mu \mathrm{mol} / \mathrm{g})$ and vegetables such as soybean $(2 \mu \mathrm{mol} / \mathrm{g})$ (Blank et al., 1992; Zeisel et al., 1986; Wurtman, 1979; Ohnishi and Fujino, 1982), yet little is known about their distribution within the body after ingestion. Only a small percentage of ingested sphingolipids are absorbed and transported into the body but work done by Silins and colleagues (2003) demonstrated that $0.1 \%$ (by weight) sphingomyelin diet reduced pre-neoplastic lesions after chemically inducing liver cancer in Sprague-Dawley rats. It had also been demonstrated (Nilsson 1968; Schmelz 1994) that rats orally fed with radiolabeled sphingomyelin and its breakdown product, ceramide ended up having some of the radiolabel in their blood, lymph and liver after a long period of time, while some ended up in the feces, somehow implying the inefficiency of the small intestine at absorbing the sphingomyelin. There was another study conducted where sphingolipid mixture was fed to rats and resulted in changes in serum lipid concentration (Imaizumi et al. 1992) such that LDL cholesterol is reduced and HDL levels are raised. Such outcomes verify their ability to pass through the intestinal mucosa and circulate systemically (Schmelz, Crall et al. 1994). More current findings in mice reveal that dietary sphingomyelin and cerebrosides pass through the stomach intact but are hydrolyzed in the small intestines, yielding choline, ceramide and sphingosine molecules. At this point, incomplete uptake of the metabolites occur with a minor fraction (10\%) of sphingomyelin passed along to the cecum and colon, where some hydrolysis can also proceed along with further uptake (Schmelz, Crall et al. 1994).

Ceramides comprise a substantial portion of the metabolites in the lumen and intestinal tissue. Ceramides and sphingosine are absorbed by intestinal cells and are either utilized for the synthesis of complex sphingolipids, which are possibly incorporated structurally in colon cells (Schmelz et al. 2000) or degraded. These metabolites by themselves are considered highly bioactive and it is thus expected that they may exert their effects on the exposed intestinal lumen cells to some extent and sphingomyelin supplementation has been shown to exhibit antitumorigenic properties (Dillehay, Webb et al. 1994). The sphingosine and some ceramides are subsequently 
absorbed by intestinal cells and degraded to fatty acids or remain in the intestine as components of complex sphingolipids (Nilsson, 1968). However, due to limited hydrolysis, approximately $25 \%$ of total ingested sphingomyelin end up getting excreted as whole (10\%), as ceramide (80-90\%) or as sphingosine (3-6\%) (Nilsson, 1968).

\subsection{Potential Anticarcinogenic Lipids}

Sphingomyelin's role as ceramide source is important since the pathway that generates ceramides from sphingomyelin is a response involved in interactions with external factors such as cytokines, stress, radiation, chemotherapeutic drugs and pathogenic substances and consequently, implicated in the regulation of cell growth, differentiation and programmed cell death. In fact, consumption of dairy products is apparently associated with a reduction in cancer risk in mice (Jarvinen et al. 2001).

In different studies, it was found that sphingosine inhibited the oncogenic transformation of normal C3H10T1/2 cells (Borek et al. 1991), the proliferation of human tumor cell lines in vitro (Stevens et al. 1990a) and in mice (Endo et al, 1991) and the metastasis of human (Endo, Igarashi et al. 1991) and mouse tumors (Sadahira, Ruan et al. 1992). It has additionally been reported that sphingosine (Stevens et al. 1990b) and ceramides (Okazaki et al. 1990) promote differentiation, which is a lost function in most transformed cell lines. This has implications on these metabolites' effects on the latter phases of carcinogenesis by reverting transformed cells into more normal phenotypes. Ceramides can even increase the sensitivity of tumors to chemotherapy (Kolesnick, 2002) and inhibit angiogenesis. Furthermore, Dillehay et al (1994) demonstrated that mice induced with colon cancer using 1,2dimethylhydrazine and subsequently fed long term with food supplemented with milk phosphosphingolipids (predominantly sphingomyelin at $0.025 \%-0.1 \% \mathrm{w} / \mathrm{w}$ sphingomyelin for 28 weeks) had reduced incidence of premalignant lesions of colon cancer (20\%) compared to controls (47\%). It is hypothesized that the anticancer effect may act by bypassing a sphingomyelinase defect (Vesper, Schmelz et al. 1999) considered to be an early indicator

of the first stages of colon cancer (Dudeja et al, 1986). Subsequent experiments further revealed that sphingomyelin caused a high percentage $(31 \%)$ of tumors to be adenomas than the more progressive malignant adenocarcinoma when fed at $0.025-0.05 \%$ of the diet, although no effect can be observed on tumor incidence or proliferation within 
40 weeks of feeding (Enkvetchakul et al. 1989). The apparent discrepancy in the effects of sphingolipids possibly parallels with findings that sphingosines may act to promote mitosis or cell proliferation (Zhang, Buckley $\boldsymbol{e t}$ al. 1990) due to their conversion to sphingosine-1-phosphate (Zhang, Desai et al. 1991). This, therefore, calls for a more cautious and in depth approach to evaluating the in vivo effects of dietary sphingolipids. Anti-cancer potential of dietary sphingolipids in skin has also been shown in a few mouse studies; the sphingoid bases inhibited induction of ornithine decarboxylase and therefore, tumor promotion (Enkvetchakul, Merrill et al. 1989) and with a specific protocol, was able to prolong the skin cancer-free period (Birt, Merrill et al. 1998).

A number of studies have contributed to elucidate particular mechanisms behind the apparent tumor suppressive effects. Hannun and colleagues (1986) found that sphingosine inhibited protein kinase C, which is assumed to play a role in cell proliferation and tumor progression (Weinstein, 1988). It is now known that ceramides participate in stress-induced apoptosis signal transduction by acting as secondary messengers of extracellular signals received via membrane protein receptors or the TNF- $\alpha$ and Fas ligand pathways (Cifone, De Maria et al. 1994). This is accomplished by blocking the cell cycle and disrupting an intrinsic apoptotic pathway. Furthermore, ceramides play key roles in epidermal differentiation, in providing a barrier for the epidermis (Geilen et al. 1997) and as proapoptotic messengers in response to stress (Burek et al. 2001).

In support of these findings, a recent discovery revealed that deregulation of sphingolipid metabolism has been correlated with certain animal diseases and possibly human cancer (Merrill et al. 1997). Sphingomyelinase activity is also reported to be a target that is inhibited in human colorectal carcinoma (Hertervig, Nilsson et al. 1997). Also, consumption of fumonisins, a group of carcinogenic toxin from fungi prevalent on agricultural products such as corn, is associated with esophageal cancer incidence in southern Africa and China (Riley et al. 1994). An enzyme involved in sphingolipid metabolism, ceramide synthase, is targeted by fumonisins, which induce cancer in the host (Wang et al. 1991). Indirect effects of sphingolipids in colon cancer inhibition have also been explored; sphingolipids were shown to induce intercellular calcium release (Ghosh, Bian et al. 1990), which in turn, is believed to be a chemoprotective agent against colon cancer (Wargovich et al. 1991).

Sphingosines have been known to promote cell survival but it is possible that they actually have a dual action as positive or negative regulators of cell growth (Spiegel and Merrill, 1996). This has been observed in different types of cells (Zhang et al. 1990; Jacobs and Kester, 1993; Stevens et al. 1990; Gomez-Muñoz et al. 1994) and is apparently dependent on the concentration of sphingosine. At low concentrations, sphingosine along with SPP 
stimulate cell growth, increase DNA synthesis and cell division (Zhang, Desai et al. 1991) presumably by mobilizing intracellular calcium molecules (Ghosh, Bian et al. 1990) and activation of the MAPK pathway (Zhang et al. 1990). Conversely, high concentrations of sphingosine have a cytotoxic effect. Sphingosine was able to induce apoptosis in HL-60 cells (Sakakura, Sweeney et al. 1996) and enhance ceramide-mediated cell death (Jarvis, Fornari et al. 1996). In general, sphingosine-1-phosphates promote tumor progression, but in a few cases also exhibit anticarcinogenic activities. Its associates with enzymes involved in removing the acetyl groups from histones to form nuclear complexes, which alter gene expression patterns leading to the upregulation of p21 (Wu et al. 1995). Therefore, the metabolites of sphingomyelin in general are conceived primarily as second messengers to a number of response pathways while capable of inducing disease at reduced levels.

Given the precursory knowledge on the potential anticancer effects of phospholipids, we sought to verify their action on skin at the molecular level by determining the extent of DNA damage and cell death in skin tissue equivalents after 24 hours of milk phospholipid incubation and subsequent UVB irradiation. Our hypothesis is that incubation of skin tissue equivalents in milk phospholipid solution prior to UV exposure reduces DNA photodamage acquired by the tissue. A $120 \mathrm{~mJ} / \mathrm{cm}^{2}$ UVB dose with intensity six times the minimal erythema dose was used to expose skin tissue grafts for the induction of photodamage. As far as we are aware, there have been no other published studies that evaluated the biological effects of milk phospholipids on UV-induced DNA damage in skin. Since little is known about the protective effects of phospholipids in skin cancer, the goals of this study are pretty basic, i.e. to determine whether treatment of skin with milk phospholipids prior to their exposure to UV would result in increased or decreased DNA damage and/or cell death. Specifically, we examined the amount of cell cycle inhibitor p21 protein expression as indicator for DNA damage and changes in nuclear morphology including DNA fragmentation and nuclear condensation. Additionally, the degree of epidermal thickening for each treatment group was noted after UV irradiation. 


\section{Chapter III: Methods}

\subsection{Milk Phospholipid Mixture}

Commercially available milk phospholipids concentrate (kindly provided by Dr. Rafael Jiménez-Flores) (PC-700, Fonterra Limited, Edgecumbe, New Zealand) was tested in this study. The mixture contains complex phospholipids with especially high levels of sphingomyelin extracted from milk and concentrated at levels 4000 times higher than that normally found in milk. Quantitative analysis of the phospholipid components in the mixture was performed by MacKenzie et al. (2009) by employing two-dimensional thin-layer chromatography (2D-TLC) and ${ }^{31} \mathrm{P}$ NMR spectroscopy techniques (Table 1). The contents of the mixture comprise of $85 \%$ lipids and $7 \%$ lactose, where phospholipids make up $60 \%$ of the total weight of the extract (Kamili et al. 2010). The principal phospholipid components include $32 \%$ phosphatidylcholine (PC), $28 \%$ phosphatidylethanolamine (PE) and $28 \%$ sphingomyelin (SM). Other phospholipid classes detected were phosphatidylinositol (PI), phosphatidylserine (PS) and dihydrosphingomyelin (DHSM) along with minor lipids lysophosphatidylcholine (LPC) and lysophosphatidylethanolamine (LPE) and the acidic phospholipid Amadori product of lactose and PE (Lac-PE). Of the fatty acid contents, $31 \%$ are oleic acids, $21 \%$ are palmitic acids and $11 \%$ are stearic acids.

Table 1. Phospholipid profile (mol\% of total phospholipids) of milk phospholipid concentrate (PC-700) analyzed with 2D-TLC and ${ }^{31}$ P NMR. Source: MacKenzie et al. (2009)

\begin{tabular}{|c|c|c|c|c|}
\hline & \multicolumn{2}{|l|}{ TLC } & \multicolumn{2}{|c|}{${ }^{31} \mathrm{P}$ NMR } \\
\hline & $\mathrm{mol} / \mathrm{s}$ & sd & $\mathrm{mol} \%$ & sd \\
\hline $\mathrm{PC}$ & 31.1 & 0.9 & 30.6 & 0.1 \\
\hline PI & 2.9 & 0.2 & 3.2 & 0.2 \\
\hline PS & 3.7 & 0.4 & 4.0 & 0.1 \\
\hline LPC & 1.6 & 0.3 & 1.8 & 0.1 \\
\hline PE & 28.0 & 0.6 & 27.9 & 0.2 \\
\hline SM & & & 21.0 & 0.1 \\
\hline DHSM & & & 5.3 & 0.2 \\
\hline DHSM + SM & 28.5 & 0.3 & 26.2 & 0.1 \\
\hline Lac-PE & 1.7 & 0.3 & 3.4 & 0.2 \\
\hline LPE & 1.8 & 0.3 & 2.9 & 0.2 \\
\hline PL in sample (\% w/w) & 53.2 & 1.1 & 60.6 & 1.5 \\
\hline
\end{tabular}


Phospholipids derived from milk are reported to be more similar in structure to human phospholipids compared to vegetable sources, which also lack a sphingomyelin component. A solution of $1 \%(\mathrm{w} / \mathrm{v})$ milk phospholipids diluted in tissue culture maintenance media (EPI-100-NMM, MatTek Corporation, Ashland, MA) was prepared for all milk-treatment samples (i.e. Milk phospholipids only and Milk phospholipids + UV groups). Around $0.1 \mathrm{~g}$ of the phospholipid powder was weighed in a conical tube and dissolved in $10 \mathrm{~mL}$ of tissue culture media by immersing the tube in a water bath set to a temperature not greater than $55^{\circ} \mathrm{C}$ for approximately 20 minutes, or until all solids are dissolved. The solution was then filter-sterilized using a $10 \mathrm{cc}$ syringe and a $0.45 \mu \mathrm{m}$ syringe filter before adding them to the milk-treatment group samples as will be discussed below.

\subsection{Sample Preparation and Incubation}

In this study, we used EpiDerm ${ }^{\mathrm{TM}}$ epidermal tissue equivalents (EPI-200, MatTek Corporation, Ashland, MA), also known as reconstructed human epidermis (RhE), consisting of highly differentiated, human-derived epithelial keratinocytes (NHEK). The lipid profile (including ceramides) of this tissue matches that found in vivo and its barrier properties includes the presence of gap junctions and approximates that of normal human skin. EpiDerm $^{\mathrm{TM}}$ has been approved by US and EU regulatory boards. Epi-200 tissue is a 3-dimensional $\left(0.63 \mathrm{~m}^{2}\right)$, multilayered in vitro model of the epidermis grown in a microporous membrane of a Millicell tissue culture insert (Figure 13) and is both metabolically and mitotically active, thereby preserving its ability to release relevant cytokines. Differentiation markers specific to the epidermis are also expressed by Epiderm ${ }^{\mathrm{TM}}$ cells (e.g. profilaggrin, K1/K10 cytokeratin repair, involucrin and type I epidermal transglutaminase). It is approximately 8-12 cell layers thick (excluding 10-15 layers of stratum corneum) and consists of the basal, spinous, granular and corneum strata, which exhibits the transition of cell shape from columnar in the stratum basale to flattened at the surface. These highly reproducible tissues are cultured at the air liquid interface, thereby exposing the surface to air. Nutrients are received from MatTek's serum-free culture medium at the bottom of the culture, therefore mimicking the oral route of nutrient delivery through the bloodstream. 


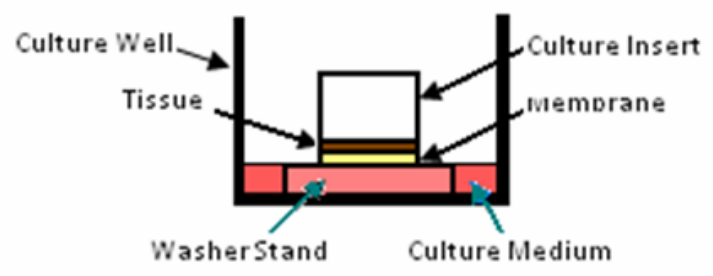

A

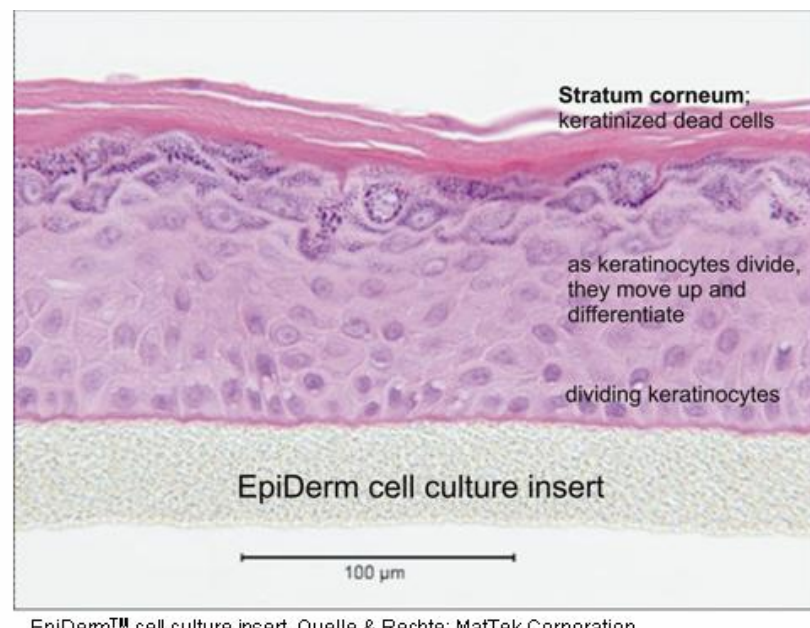

EpiDem $^{\top \mathrm{IM}}$ cell culture insert. Quelle \& Rechte: MatTek Corporation

B

Figure 13. EpiDerm ${ }^{\mathrm{TM}}$ reconstructed human epidermis (RhE) tissue model with cell culture insert. Source: MatTek Corporation, (2007).

Before the arrival of the tissues, all materials used for handling the tissue culture, such as forceps and scissors, were autoclaved. Upon receipt, tissues were placed in the refrigerator right away as culture materials were prepared. The maintenance (EPI-100-NMM, MatTek Corporation, Ashland, MA) and assay media (EPI-100-ASY, MatTek Corporation, Ashland, MA) were initially aliquoted and filter-sterilized onto a $50 \mathrm{~mL}$ Falcon conical tube then pre-warmed in a $37^{\circ} \mathrm{C}$ water bath prior to use. Under sterile conditions in the laminar flow hood, each well of a 6-well plate was filled with $0.9 \mathrm{~mL}$ of pre-warmed assay media and labeled completely according to their designated treatments. It was necessary to pre-incubate the tissues at $37^{\circ} \mathrm{C}, 5 \% \mathrm{CO}_{2}$ for 1 hour or overnight so as to aid in cell recovery and the release of transport-stress related compounds.

The tissue samples were divided into four treatment groups: a "Control" group received no UV and no milk phospholipid treatment, a "UV" group was incubated with maintenance media prior to UV exposure, a "Milk Phospholipids" group was treated with milk phospholipids alone, and lastly, a "UV + Milk Phospholipids" group was treated with milk phospholipid incubation before UV exposure. Sample tissues were taken from the refrigerator and the package was sterilized with $70 \%$ ethanol. The package was opened and without touching the sterile inner surface, sterile forceps were used to remove the gauze. Then, each culture insert was separated from the mediuminfused agarose gel while still cold. Performing this step at low temperature was crucial to facilitate separation of the agarose gel adhering to the inserts and care was taken to remove any remaining adhering agarose gel by blotting the inserts onto the sterile gauze. The inserts were then transferred onto empty, sterile 24-well plates for visual 
inspection within the next 5 minutes. Tissue defects that were noted include excess surface moisture and discoloration. Tissues were subsequently transferred to the pre-filled 6-well plates, taking care to avoid the formation of bubbles. Tissues were then incubated at $37^{\circ} \mathrm{C}$ and $5 \% \mathrm{CO}_{2}$ for 1 hour.

After 1 hour pre-incubation, each of four tissue samples was then transferred onto a well of a 6-well plate pre-filled with $5 \mathrm{~mL}$ of either fresh maintenance media for Control and UV samples, or $1 \%$ milk phospholipid solution for UV + Milk Phospholipids and Milk Phospholipids samples over washer stands (EPI-WSHR, MatTek Corporation, Ashland, MA). Lastly, the tissues were incubated at $37^{\circ} \mathrm{C}$ and $5 \% \mathrm{CO}_{2}$ for 24 hours.

\subsection{UV Irradiation}

After 24 hours of incubation, the tissues were prepared for exposure to intense UV dose. At first, 6-well plates with $5 \mathrm{~mL}$ maintenance media were prepared and washers were placed on each well using aseptic technique under the hood. The plates with media were then warmed in the incubator. After hands were sprayed thoroughly with sunscreen, the UVP EL Series UVL-28 lamp (UVP, LLC, Upland, CA) was wiped down and sprayed with 70\% ethanol, then warmed up by turning the power on for about 5 minutes. With the room lights turned off, the average intensity of the lamp was measured using a ThorLabs Optical Power Meter (ThorLabs, Newton, NJ) by reading the UV wattage at the corner of the lamp base and along the center of the base where the lamp emits the highest wattage. The time of exposure for a $120 \mathrm{~mJ} / \mathrm{cm}^{2} \mathrm{UV}$ dose was then calculated using the area of the detector, 0.7088 $\mathrm{cm}^{2}$ in our case, and the average wattage reading acquired previously. Sample computations are as follows:

\footnotetext{
Average wattage $=\frac{\text { corner wattage }+ \text { center wattage }}{2}=\frac{240 \mu \mathrm{W}+282 \mu \mathrm{W}}{2}=261 \mu \mathrm{W}=\mathbf{0 . 2 6 1} \mathbf{~ m J} / \mathbf{s}$

Exposure time $=\underline{(\text { desired dose })(\text { area of detector })}=\frac{\left(120 \mathrm{~mJ} / \mathrm{cm}^{2}\right)\left(0.7088 \mathrm{~cm}^{2}\right)}{0.261 \mathrm{~mJ} / \mathrm{s}}=325.89 \mathrm{sec}=\mathbf{5} \mathbf{~ m i n}, 26 \mathrm{sec}$ average wattage $0.261 \mathrm{~mJ} / \mathrm{s}$
}

A tissue sample was then placed at the center of an empty, sterile $100 \mathrm{~mm}$ petri dish with the cover removed. The center of the dish was then positioned with the tissue right under the highest UV intensity as measured previously. In the dark, the UV lamp was set to $302 \mathrm{~nm}$ and the tissue was exposed to UV for the calculated time to give a total dose of $120 \mathrm{~mJ} / \mathrm{cm}^{2}$. 


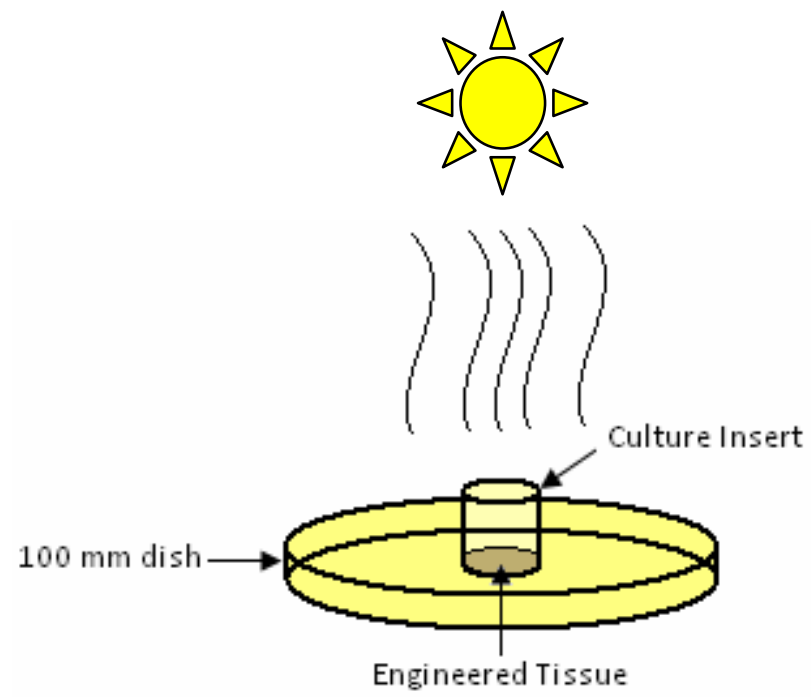

Figure 14. Set-up for UV irradiation of tissue engineered skin sample.

Following exposure, the tissues were immediately transferred to a 6-well plate pre-filled with $5 \mathrm{~mL}$ of fresh maintenance media, while avoiding bubbles, and placed in the $37^{\circ} \mathrm{C}$ incubator. UV exposure and incubation were then performed as described for the remaining tissues designated to receive UV treatment. Tissues that were not irradiated with UV were also transferred to wells containing fresh media and all tissues were incubated at $37^{\circ} \mathrm{C}, 5 \%$ $\mathrm{CO}_{2}$ for another 24 hours.

\subsection{Fluorescence and Immunofluorescence Microscopy}

\subsubsection{Tissue Fixation and Chemical Processing}

Approximately 24 hours after UV exposure, several tissues were set aside for histological examination. First, tissues were excised from the culture insert using a sterile scalpel blade. The tissues were then fixed to preserve the integrity of the cell structure and antigenic sites by submerging in Histochoice for at least 30 minutes until tissues are ready to be processed further. Samples were then loaded onto a chemical processor. After at least half an hour, samples were placed upright on a metal tray and melted paraffin wax was dispensed onto the tray to encase the tissue. The bottom half of a plastic cassette was then placed carefully on top of the dispensed wax while avoiding dislodging the tissue from its upright position. The paraffin embedded samples were then cooled for at least an hour to solidify the wax and stored at room temperature until further processed for microtomy. 


\subsubsection{Microtomy}

For the microtomy procedure, $6 \mu \mathrm{m}$ histological sections were obtained to facilitate staining and visualization of cellular morphology with microscopy. To prepare for sectioning, samples were incubated at $4^{\circ} \mathrm{C}$ for at least 20 minutes to facilitate easy tissue sectioning and prevent premature melting of the paraffin wax during slicing. The tissue flotation bath was then half-filled with water and plugged into the outlet. The paraffin-embedded sample was placed on the tissue holder of the microtome and clamped securely. While wearing gloves, the microtome blade was fitted and locked into position and the tissue was lowered to the level of the blade by unlocking and turning the hand wheel clockwise. The blade, mounted on its base, was then moved towards the tissue block until it was close enough to almost touch the flat surface of the block. The base was then locked into place and the tissue slice setting was adjusted to around 15 microns. The hand wheel was rotated clockwise to produce tissue slices until the slices obtained contained tissue sections within the center of the tissue sample. The slice setting was then adjusted to 6 microns and the succeeding $6 \mu \mathrm{m}$ slices were placed on the water surface of the flotation bath using forceps. Adjacent tissue slices were separated in two's by poking the section edges with forceps and two block slices were scooped onto the top of a coated glass slide from underneath the water surface. The slide was then placed in the microscope slide rack. The procedure was repeated for sample replicates and for the each of the blocks for the other treatment groups.

\subsubsection{Deparaffinization and Rehydration}

Deparaffinization and rehydration steps are crucial to prevent poor staining of the specimen due to the incomplete removal of paraffin wax or inadequate penetration of stains into the tissue. The slide rack containing the glass slides were placed in an incubator or oven and heated to $55^{\circ} \mathrm{C}$ to allow the tissues to attach to the slides until the paraffin wax melted and turned clear ( 15 minutes). At this point, the immunohistochemistry reagent baths were prepared for the following incubation steps: 2 Xlyene baths, two $100 \%$ ethanol baths, one $95 \%$ ethanol bath and 1 distilled water bath. As the wax melted and became clear, the slide rack was immersed in the first Xylene bath for 5 
minutes inside the fume hood. The rack was then transferred into the second Xylene bath and incubated for another 5 minutes. Afterwards, the rack was submerged in the first $100 \%$ ethanol bath for a total of 2 minutes followed by another 2-minute immersion in a $100 \%$ ethanol bath. The rack was then transferred to the $95 \%$ ethanol bath for 1 minute and lastly, to the distilled water bath for another minute.

\subsubsection{Permeabilization, Blocking and Staining}

The slides were taken out of the slide rack and placed on top of a paper towel. Triton-X detergent was prepared at $10 \%(\mathrm{v} / \mathrm{v})$ concentration in distilled water to permeabilize the tissue and allow easy penetration of the stains into the nuclear membrane. A volume of $200 \mu \mathrm{L}$ of $10 \%$ Triton-X solution was dispensed on top of the specimen for each slide and allowed to sit at room temperature for 50 minutes. The slides were then rinsed twice with PBS. BSA at $1 \%(w / v)$ concentration was prepared and used for the subsequent blocking step. Likewise, 200 $\mu \mathrm{L}$ of $1 \%$ BSA for each slide was dispensed on top of the specimen and allowed to sit at room temperature for 50 minutes. The slides were again washed with PBS twice before staining. The primary antibody used was Alexa Fluor ${ }^{\circledR}$ 647-conjugated p21 (F-5) (Santa Cruz Biotechnology, Santa Cruz, CA) diluted at 1:10 with 1\% BSA. Around $200 \mu \mathrm{L}$ of the antibody was placed on top of the tissues for each slide. The slides were then incubated overnight at $4^{\circ} \mathrm{C}$. The next day, slides were rinsed twice with PBS and $1 \mu \mathrm{g} / \mathrm{mL}$ Hoechst 34580 was prepared by diluting in PBS. Hoechst dye is a stain that readily permeates the cell membrane and binds to the minor groove of the DNA (Figure 15) with high selectivity for AT-rich sequences to emit a bright blue fluorescence. Each slide was incubated at room temperature with $200 \mu \mathrm{L}$ of the prepared Hoechst dye for a total of 15 minutes, while covered in aluminum foil. The slides were then washed twice with distilled water, mounted with coverslips and allowed to dry overnight. 


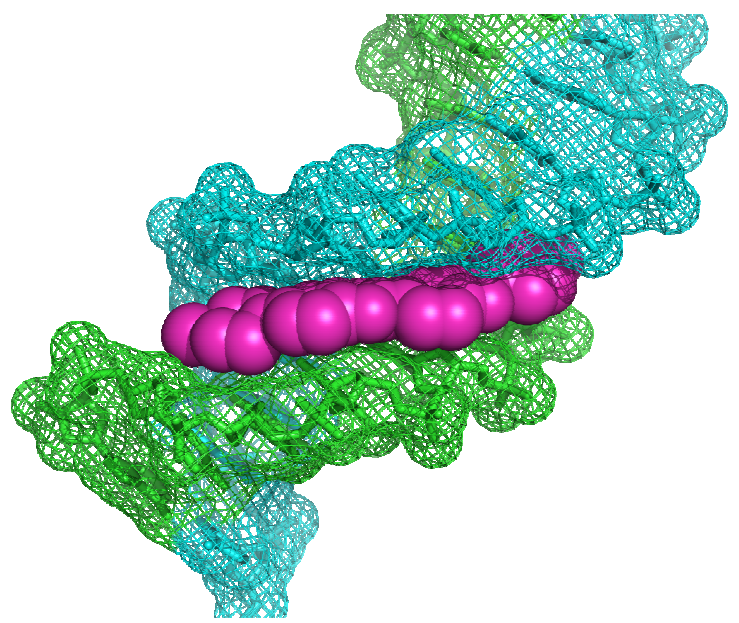

Figure 15. Image of the Hoechst dye binding to the minor groove of DNA. Source: Vega et al. (1994).

\subsubsection{Laser Scanning Confocal Fluorescence Microscopy}

Confocal microscopy has a wide range of advantages over conventional widefield fluorescence microscoppy. These include the ability to acquire extremely high-quality images at controlled depths of field, dramatic reduction of background from regions away from the focal plane and 3D reconstruction of thin $(0.5-1.5$ $\mu \mathrm{m})$ serial optical sections from thick specimen. These are made possible by spatial filtering of out-of-focus light including secondary fluorescence emitted from excited volumes of the specimen, which is often an issue that compromises the resolution in widefield epi-fluorescent microscopes.

The peak absorption and emission wavelengths of Hoechst 34580 dye when bound to DNA are at $392 \mathrm{~nm}$ and $440 \mathrm{~nm}$ respectively (Figure 16). The excitation wavelength of the DAPI filter is around $360 \mathrm{~nm} / 405 \mathrm{~nm}$ while the emission is around $460 \mathrm{~nm}$. Hoechst dye has a relatively large Stokes shift or gap between its excitation and emission maxima and consequently, has small overlap between the absorption and emission spectra. Since the crucial aim of fluorescence microscopy is to irradiate the specimen with a specific range of wavelengths and separate the weaker fluorescence emission from the excitation light, this large gap ensures that the signal detected is mostly coming from emitted fluorescence from the tissue as opposed to signal from the excitatioin light. Thus low background staining is often observed during visualization of Hoechst staining. 


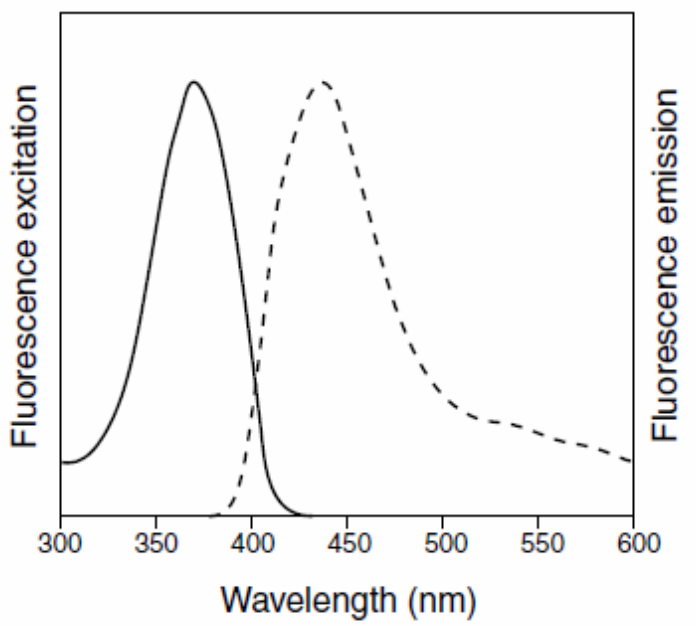

Figure 16. Approximate fluorescence excitation and emission of Hoechst 34580 dye. Source: Invitrogen, (2005)

The stained tissues were visualized with an Olympus FluoView Laser Scanning Confocal Microscope (FV100, Olympus America, Centerville, PA). A drop of immersion oil was placed on top of the objective lens and the slide was clamped onto the microscope stage with the top side facing down. The specimen was initially located using the brightfield setting and the 20X objective. The lens was moved upwards until it touched the slide's bottom surface. The focus was adjusted using the coarse and fine adjustment knobs to localize the tissue. After acquiring a clear focus of the specimen, the light source was shifted to widefield fluorescence illumination, which activated the lasers. Using the FluoView software, Hoechst staining was visualized by selecting the DAPI filter settings and the p21 staining was visualized using the Alexa Fluor 647 filter. Samples were then scanned in both channels simultaneously by clicking on "XY Repeat". When the desired image has been acquired the scanning is stopped by clicking the "Stop" button and all channels of the image files are saved as .oib files. All saved files are then exported as .tif files. 


\subsubsection{Image Analysis}

\subsubsection{Experimental Design}

In order to remove any conscious or subconscious bias during subsequent analyses of the images, a blind study was devised where information about the image sources, in terms of the treatment groups they belong to, the date of acquisition and microscope settings used were concealed from the analyst. Prior analysis of DNA fragmentation was conducted by three analysts in a non-blinded study but due to time constraints the thickness measurements, percent p21 staining and repeat DNA fragment analyses were conducted blindly by only one analyst. An arbitrary population of at least 200 images from different experiment dates, usually involving different paraffin blocks and conducted by different researchers ${ }^{1}$ but using and following the same experimental variables and protocols were obtained. These images were then assigned random numbers as their filenames and placed in a single folder to conceal as much information prior to proceeding with image analysis.

The ideal sample size for image analysis was determined by obtaining the standard error from a pilot study which involved the counting of DNA fragments in six images of each Control and UV sample groups. Using the Minitab software, a normality (State $\rightarrow$ Basic Statistics $\rightarrow$ Normality Test) and equal variance test (Graph $\rightarrow$ Scatterplot $\rightarrow$ Simple) was first conducted since the sample size was less than 30; a p-value of more than 0.05 indicates a normally distributed sample. An ANOVA test was run using Control and UV sample \% DNA fragments data by selecting the General Linear Model test (Stat $\rightarrow$ ANOVA $\rightarrow$ General Linear Model). In the General Linear Model dialog box, the "\% Fragments" column header for the \% DNA Fragment values was entered in the "Responses" field, while the column header "Treatment" for the treatment groups was entered in the "Model" field (Figure 17).

\footnotetext{
${ }^{1}$ Experiments were conducted in collaboration with C. Dargitz, M. Bingham and A. Russell of the Biomedical Engineering \& General Engineering Department, California Polytechnic State University, San Luis Obispo, CA.
} 


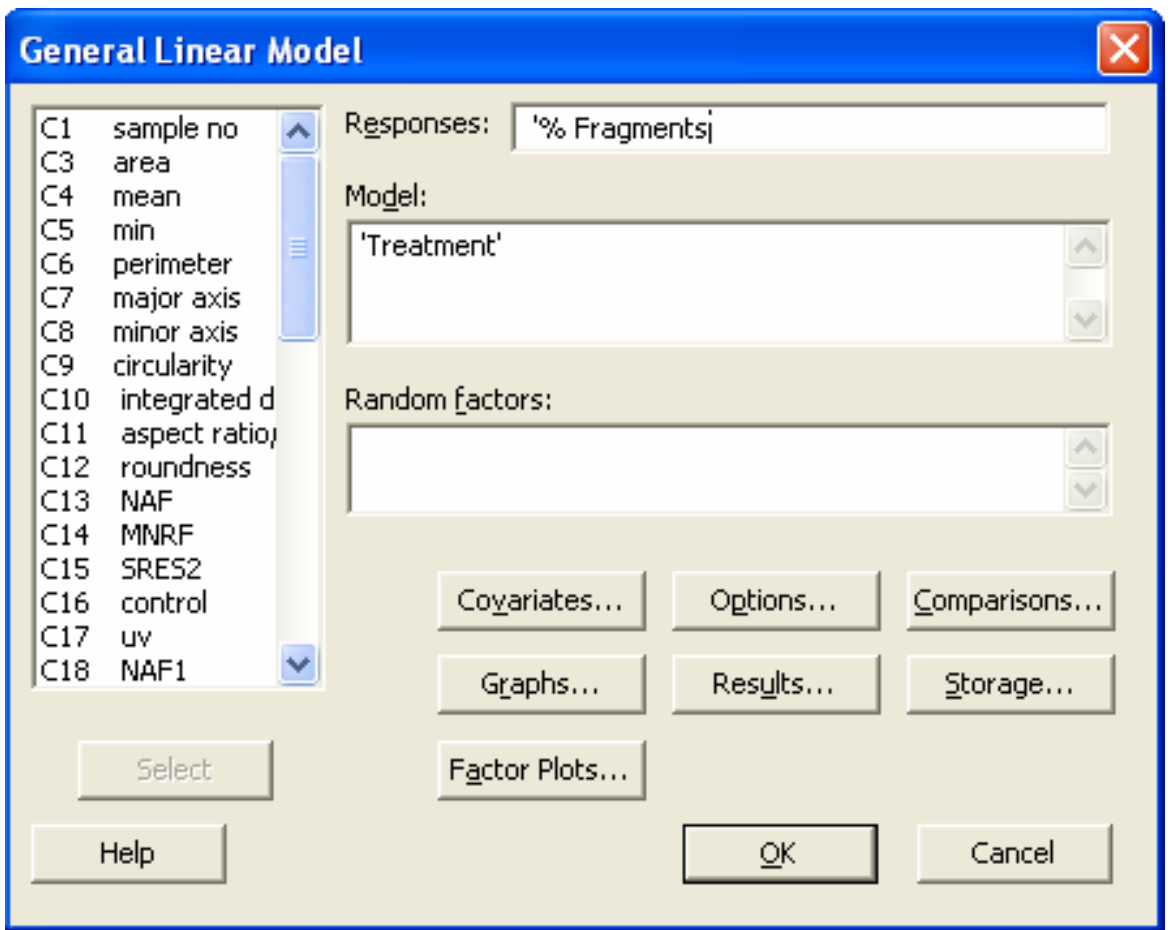

Figure 17. Minitab General Linear Model dialog box entries for obtaining the standard error for the pilot study.

The standard error obtained from this step was then used to run a 2-sample t-test (Stat $\rightarrow$ Power and Sample Size $\rightarrow$ 2-Sample $t$ ) for estimating the power and sample size for the experiment. An effect size of 8 was chosen for the "Differences" and a minimum power of 0.8 (80\%) was entered in the "Power values" field (Figure 18).

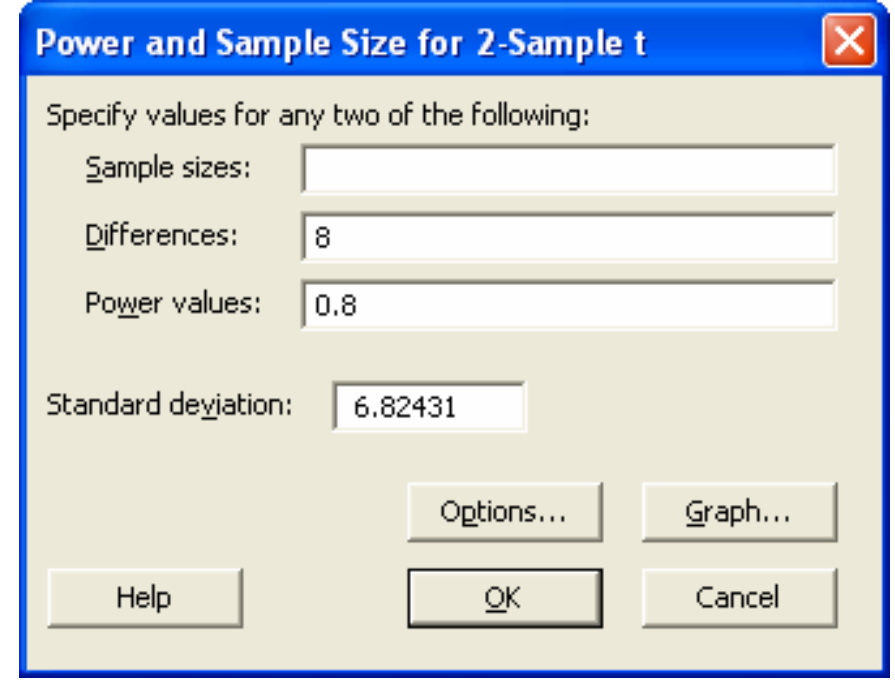

Figure 18. Power and Sample Size values for determining the ideal sample size for \% DNA fragment counting. 


\subsubsection{Setting the Measurement Scale}

The images were analyzed using ImageJ, a Java-based image processing program commonly used for biological image analysis, which can be downloaded for free from the NIH. The image files are opened in ImageJ by dragging and releasing the file icon into the program taskbar. The measurement scale was then set by first drawing a straight horizontal line from the left edge to the right edge of the image using the straight line tool (Figure 19). The Set Scale option was then selected (Analyze $\rightarrow$ Set Scale...) to open the Set Scale dialog box (Figure 20). The software for the Confocal Microscope provided the reference values for the image size ( 800 pixel x 800 pixel) and its corresponding scale value $(0.795 \mu \mathrm{m} / \mathrm{pixel})$.

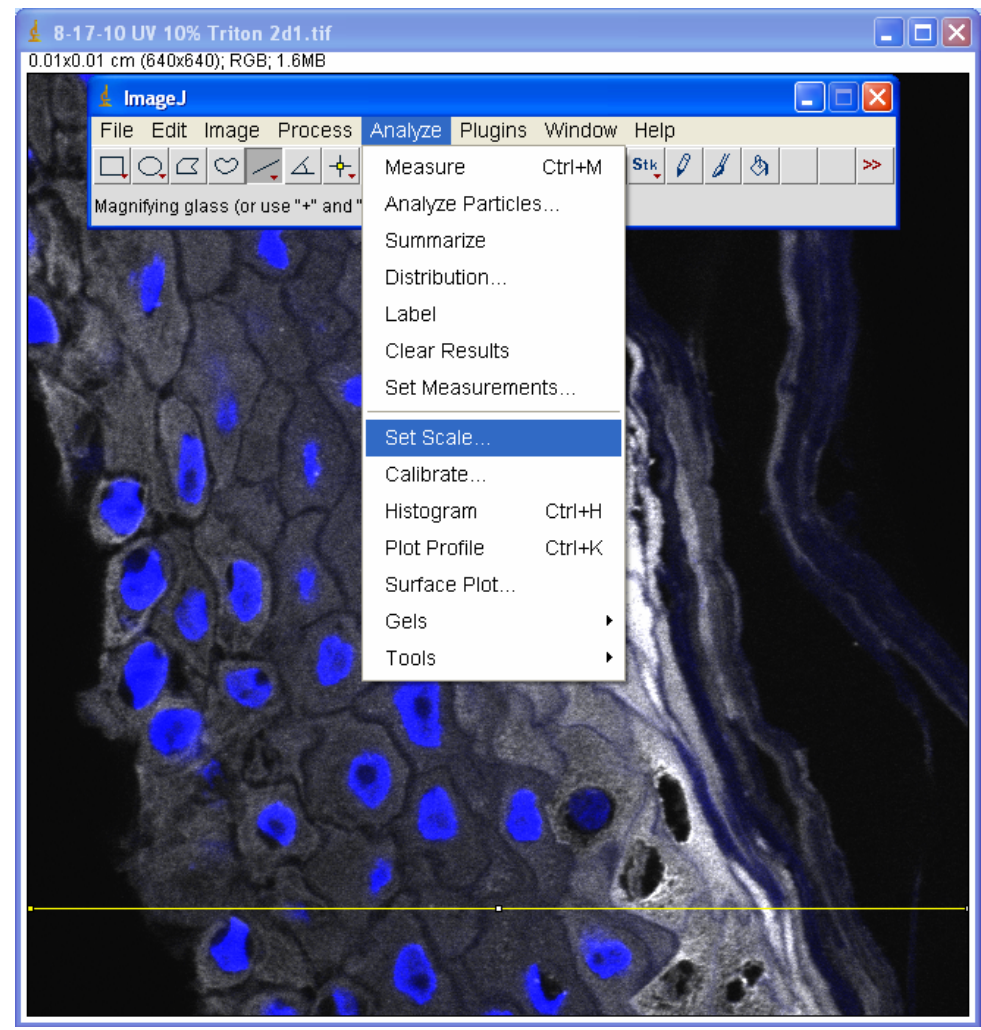

Figure 19. Setting the measurement scale using the straight line tool and set scale option. 


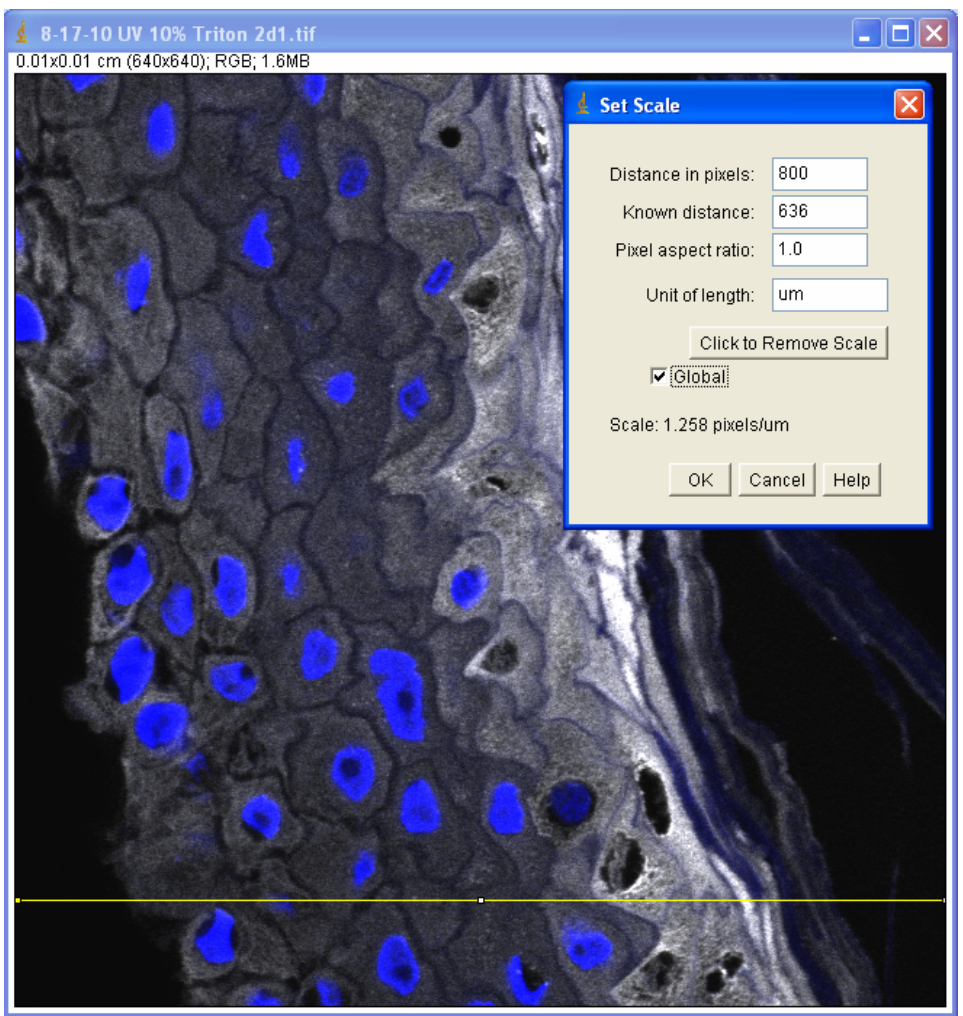

Figure 20. Dialogue box entries for modifying set scale settings for the image.

The following describes the values entered in the dialogue box for setting the scale:

Distance in Pixels: 800

Known Distance: 800 pixels x $0.795 \mu \mathrm{m} /$ pixel $=\mathbf{6 3 6} \boldsymbol{\mu m}$

Units: $\mu \mathrm{m}$

The box for Global was ticked to allow the settings to carry over to all succeeding file images that will be opened. The resulting scale conversion was double checked by comparing the value with the reciprocal of 0.795 $\mu \mathrm{m} /$ pixel (1.25786 pixel/ $\mu \mathrm{m})$. The "OK" button was then clicked to save the changes.

\subsubsection{Measurement of Epidermal Thickness}

The thickness of the epidermis was measured using the straight line tool in ImageJ. A total of 26 images from each treatment group were analyzed for the thickness measurements. Each file was opened and the image was rotated until the basement membrane aligned parallel to the x-axis. The straight line tool was then used to draw a vertical line from the basement membrane to the top surface of the stratum corneum and the length of the line was 
recorded (Figure 21). A second vertical line was drawn in another section of the tissue and the average of the two line measurements was used as the thickness measurement for each image.

An ANOVA test was performed using Minitab software to find out if the mean values for the thickness measurements between the treatment groups were equal. Data from all images were stacked in a single column and entered as the "Response" variable. Another column containing the corresponding treatment group for each measurement was designated as the "Model" variable in the General Linear Model dialog box. Tukey's pairwise comparison method was used in conjunction to ANOVA to reveal which among the means are significantly different from the others. Additionally, the individual effects of each treatment factor (i.e. milk phospholipid incubation and UV exposure) on epidermal thickening were analyzed by running the main effects and interactions plot; in the General Linear Model dialog box, the treatment factors were entered in the "Factors" field under the Main Effects as well as the Interactions Plot.

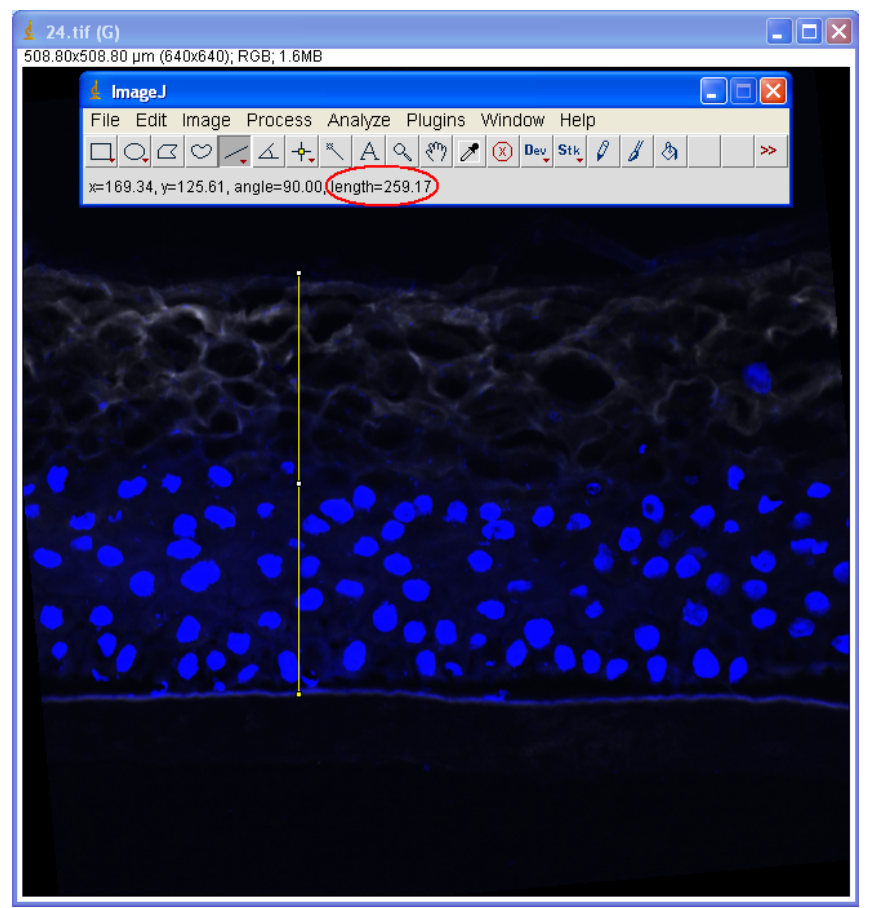

Figure 21. Epidermal thickening measurement using the straight line tool of ImageJ. 


\subsubsection{Percent DNA Fragment Analysis}

The cell counter plug-in (Plugins $\rightarrow$ Analyze $\rightarrow$ Cell Counter) in ImageJ was used to count individual cells with fragmented DNA (Figure 22). A total of 19 images were analyzed for each treatment group. Valid counts included nuclei that have jagged outlines, holes and variable intensities. Half-moon nuclei were identified as nuclear condensation. Fragments that were clumped together were counted as one. Excluded from the cell counts were cells that were out of focus and those located on the margins and tissue defects.

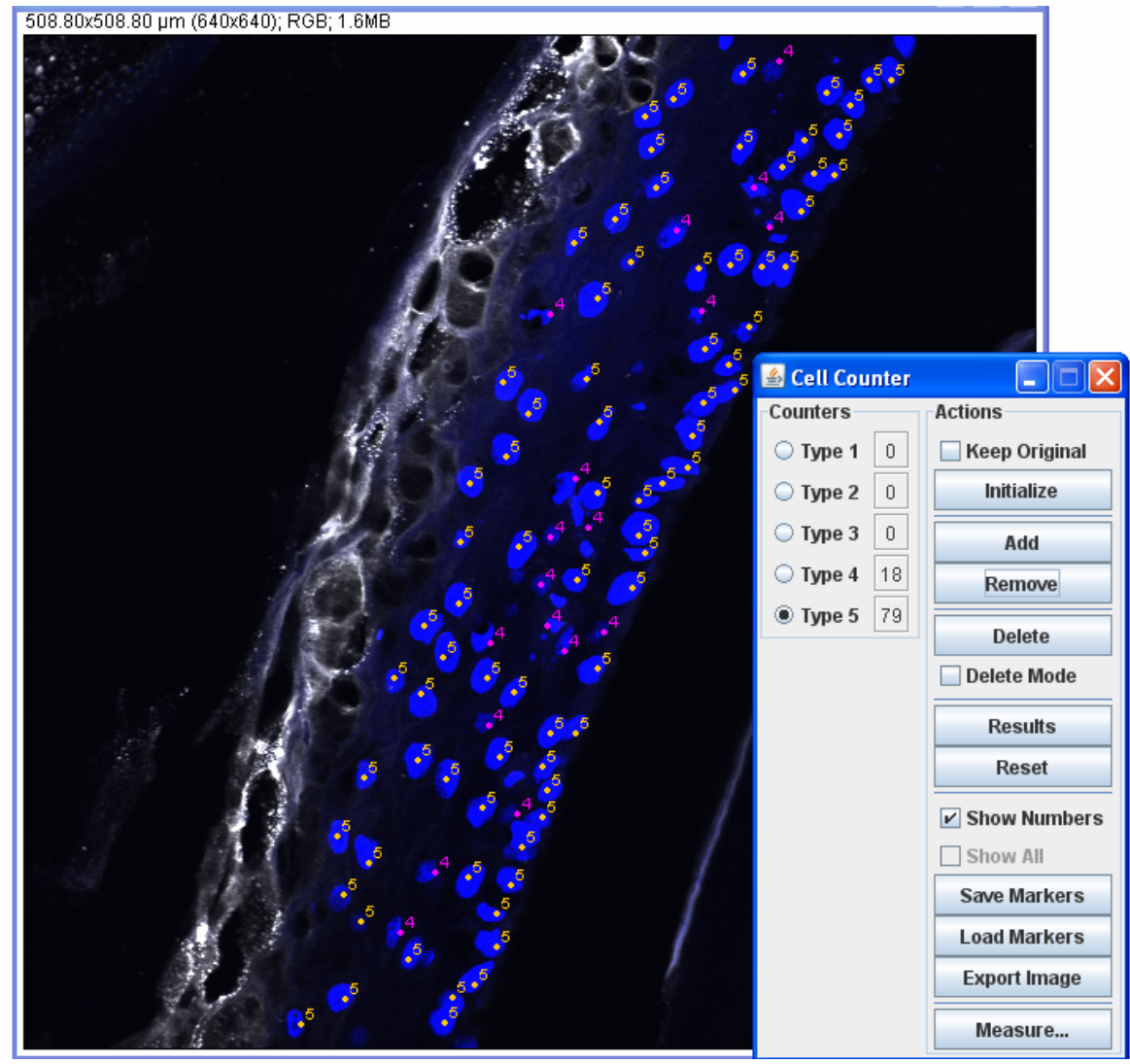

Figure 22. ImageJ cell counter plug-in for \% DNA fragment measurements.

Likewise, individual \% DNA fragment values were entered in Minitab and an ANOVA with Tukey's comparison test was conducted to determine significant differences as described previously. In addition, the interaction plot between the two treatment factors was obtained. 


\subsubsection{Analysis of $\mathbf{p} 21$ Immmunofluorescence-Positive Cells}

The number of cells that were positive for p21 staining was also counted using the cell counter plug-in. A total of 17 images for each treatment was used for this study. Initially, the DAPI and Alexa ${ }^{\circledR}$ Fluor 647 filter channel images were opened in ImageJ. The total cell count was determined from the DAPI channel image and recorded. Both files were rotated to align the basement membrane to the $\mathrm{x}$-axis then each was converted to an 8-bit image (Image $\rightarrow$ Type $\rightarrow$ 8-bit). The DAPI and Alexa ${ }^{\circledR}$ Fluor 647 channel images were then converted to blue and red pseudocolors respectively from the "Lookup Tables" options (Image $\rightarrow$ Lookup Tables) (Figure 23). The image files were then merged using the "AND" function of the image calculator such that regions with staining in both channels appeared in the third image (Process $\rightarrow$ Image Calculator $\rightarrow$ “AND”) (Figure 24). Again from the "Lookup Tables", the gamma, intensity and contrast of the image was changed to "brgbcmyw" (Image $\rightarrow$ Lookup Tables $\rightarrow$ brgbcmyw), resulting in an image of cells outlined in red with bright green p21 staining. Outlined cells containing p21 staining were counted as p21-positive cells and their percentages were calculated.

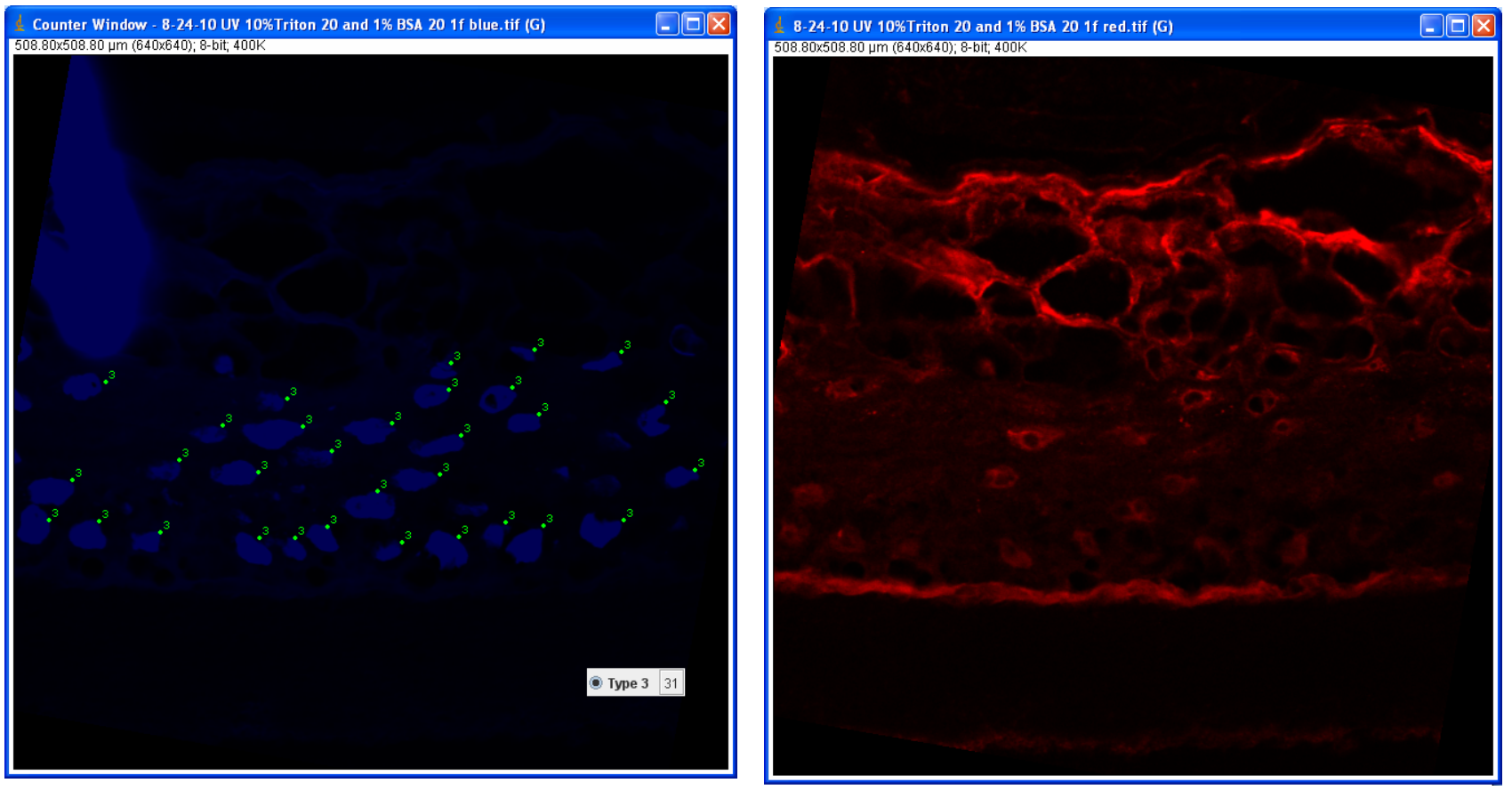

Figure 23. Blue and red channel of a UV sample image. Images were rotated, transformed to 8-bit images and assigned blue and red color from the Lookup Table in imageJ. 


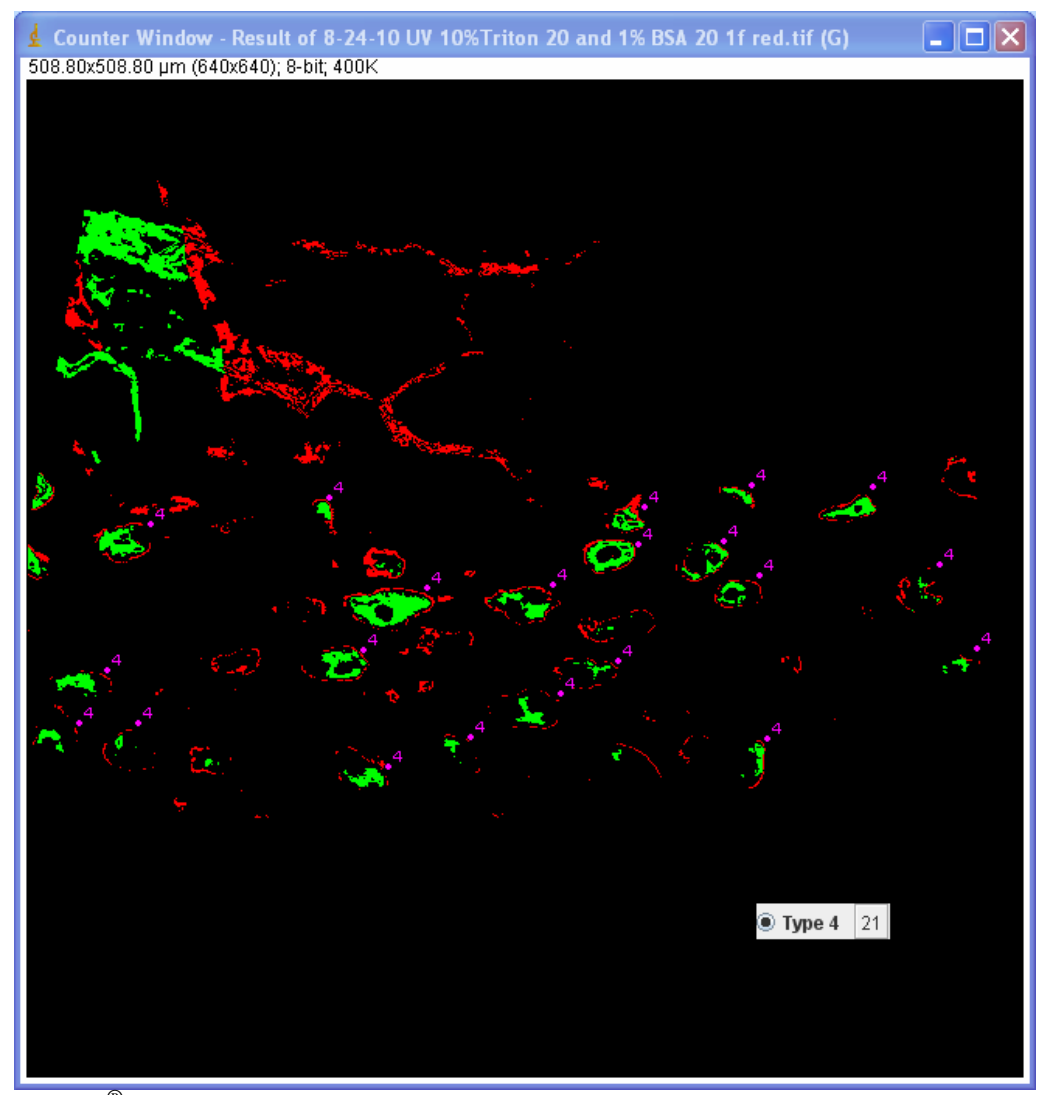

Figure 24. DAPI and Alexa ${ }^{\circledR}$ Fluor 647 channels merged using the image calculator "AND" function then converted to "brgbcmyw" LUT. Cells with green p21 staining were counted with the cell counter plug-in. Note that background staining at the top left corner is not eliminated with this method.

\subsection{Western Blot}

\subsubsection{Tissue Lysis and Total Protein Extraction}

Protein extraction is a very crucial step in the success of the Western blot procedure since the downstream processes depend so much on the quantity and quality of the protein yielded from this procedure. The efficacy of the extraction method involves four critical steps in the procedure: effective tissue and cell disruption, release of proteins into soluble form and from possible binding with other molecules, inactivation of proteinases and purification from contaminating agents including nucleic acids, carbohydrates and lipids. All protein extraction procedures were performed on ice to inactivate proteases and therefore, inhibit degradation of proteins especially after their release from cells. 
Various extraction protocols and lysis buffers were tested to determine which method would effectively disrupt the robust skin tissue and give maximal protein yield: urea extraction, TCA/acetone buffer, $1 \%$ NP-40, $2 \%$ SDS extraction and finally, 2\% SDS extaction with TCA/acetone precipitation. Urea is a strong chaotropic agent that solubilizes proteins via hydrogen bonds and electrostatic interactions (Moglich et al. 2005). NP-40 is a nonionic detergent along the same detergent classification as Triton X-100. These agents are considered to be mild and act by disrupting lipid-lipid and lipid-protein interactions. TCA/acetone on the other hand, is a delipidation agent that selectively solubilizes lipids and thus, frees them from proteins, which are precipitated out of the solution. Ultimately however, 2\% SDS extraction procedure with TCA/acetone precipitation was the most efficient at extracting the highest yield of total proteins.

On the day following UV exposure, all tissues were harvested and processed for protein extraction immediately. First, tissues were excised from their culture inserts using a sterile scalpel blade and immediately placed onto a $1.2 \mathrm{~mL}$ screw-cap tube with round bottom. A volume of $360 \mu \mathrm{L}$ of the ice-cold lysis buffer being tested (see Appendix for recipe) was added to the $10 \mathrm{mg}$ tissue sample, which was subsequently macerated with around 50 strokes of a tight-fitting Wheaton Dounce homogenizer piston. The tissue pieces were then homogenized further using an IKA Ultra-Turrax T8 homogenizer (Janke and Kunkel, Staufen, Germany) for 15 seconds at maximum speed. This was repeated until no clumps of tissue remained visible and the tubes were kept on ice in between bursts. Tissue samples were then placed in an orbital shaker for a total time of 1 hour and agitated by vortexing occasionally. Alternatively, a 25-29 G syringe was used to further homogenize the lysate and ensure nuclear membrane lysis. A $10 \mu \mathrm{L}$ volume was aliquoted at this point and placed on a glass slide. The specimen was then stained with $1 \mu \mathrm{g} / \mathrm{mL}$ Hoechst 34580 dye (Invitrogen, Carlsbad, CA) and visualized under the DAPI filter of an Olympus FluoView Laser Scanning Confocal Microscope (FV100, Olympus America, Centerville, PA) at 40X magnification to check for any intact nuclei. The rest of the lysates were centrifuged at 12,000 $\mathrm{x} g$ for 15 minutes at $4^{\circ} \mathrm{C}$. The resulting supernatant, which contains the extracted proteins, was then transferred to a fresh, labeled microcentrifuge tube and stored at $-20^{\circ} \mathrm{C}$, while a second round of lysis steps was repeated on the pellets with another $360 \mu \mathrm{l}$ volume of the lysis buffer. The resulting suspension was again vortexed and homogenized. Afterwards, the tissue samples were again spun at $12,000 \times \mathrm{g}$ for 15 minutes at $4^{\circ} \mathrm{C}$. Once more, the supernatant was collected and added to the first tube of supernatant, while the remaining pellet was discarded. 
In the latter period of protein extraction protocol optimization, a Trichloroacetic acid (TCA)/acetone precipitation procedure was additionally performed on the collected supernatant after every extraction procedure, so as to concentrate the proteins as well as purify them against contaminants that may interfere with the succeeding steps. In advance, sufficient volume of precipitation media (see Appendix for recipe) was cooled to $-20^{\circ} \mathrm{C}$. Then, to the supernatant from the extraction step, precipitation media was added at a volume four times that of the supernatant. The tubes were then vortexed and incubated at $-20^{\circ} \mathrm{C}$ overnight. The following day, tubes were centrifuged at 22,000 $\mathrm{x} g$ for 60 minutes. This time, the supernatant was discarded and the pellet was resuspended with the same volume of the wash buffer (see Appendix for recipe). The tubes were again centrifuged at 22,000 x $g$ for 60 minutes and the supernatant was discarded. Finally, the pellet was either air dried or dried using an Eppendorf Vacufuge ${ }^{\circledR}$ before resuspending the pellet in $160 \mu 1$ of Tris-Buffered Saline (TBS) (see Appendix for the recipe).

\subsubsection{Protein Quantification}

The protein yield from the extraction procedure was determined by an absorbance assay known as the Bradford Assay. This method involves the use of protein standards of known concentration in order to generate a standard curve and facilitate intrapolation of an unknown sample's protein concentration. For this method, the microassay procedure, appropriate for smaller protein volumes, was performed. To begin with, protein standards of increasing concentration were prepared from the same stock solution of $1 \mathrm{mg} / \mathrm{mL}$ Bovine Serum Albumin (BSA) as shown in Table 2.

Table 2. Designated volumes of Bradford Assay protein standards from a $1 \mathrm{mg} / \mathrm{ml}$ BSA standard stock solution.

\begin{tabular}{|c|c|c|c|}
\hline Test Sample (working concentration) & Vol. BSA Stock $(\mu \mathrm{L})$ & Vol. TBS buffer $(\mu \mathrm{L})$ & $\begin{array}{c}\text { Vol. Bradford } \\
\text { Reagent }(\mu \mathrm{L})\end{array}$ \\
\hline Blank & 0 & 800 & 200 \\
\hline BSA Standard $(4 \mu \mathrm{g} / \mathrm{mL})$ & 4 & 796 & 200 \\
\hline BSA Standard $(16 \mu \mathrm{g} / \mathrm{mL})$ & 16 & 784 & 200 \\
\hline BSA Standard $(27 \mu \mathrm{g} / \mathrm{mL})$ & 27 & 773 & 200 \\
\hline BSA Standard $(35 \mu \mathrm{g} / \mathrm{mL})$ & 35 & 765 & 200 \\
\hline
\end{tabular}

Samples and standards were first incubated at room temperature and the SPECTRAmax PLUS384 UV/Vis spectrophotometer (Molecular Devices, Sunnyvale, CA) was warmed up for about 15 minutes. The reading 
wavelength for the spectrophotometer was set to $595 \mathrm{~nm}$, which is specific to detecting the absorbance maximum shift of Coomassie Blue G-250 dye from $465 \mathrm{~nm}$ to $595 \mathrm{~nm}$ upon binding with proteins in acidic solution. Next, the corresponding volume of TBS buffer and Bradford Reagent (see Appendix for recipe) was pipetted onto a $1 \mathrm{~mL}$ quartz cuvette. This reference cuvette is then placed onto the chamber for the UV light path and read by pressing the "Ref" button to set up the blank as the baseline reference reading. The absorbance measurement of each BSA standard was then measured twice and plotted as a function of its theoretical concentration, which should give a linear curve.

According to the Beer-Lambert Law, there is a linear relationship between absorbance and protein concentration such that:

$$
\mathbf{A}=\mathbf{\varepsilon c l},
$$

where $\boldsymbol{\varepsilon}$ is the molar extinction coefficient for the absorbing material at the specific wavelength $(595 \mathrm{~nm})$ in units of $\mathrm{L} /(\mathrm{mol} \mathrm{x} \mathrm{cm})$; $\mathbf{c}$ is the concentration of the absorbing solution in molar $(\mathrm{M})$ units; and $\mathbf{l}$ is the path length of light in the absorbing material, which is $1 \mathrm{~cm}$ in our case. The best-fit line was then drawn as a straight line through the data points from the BSA standards measurement and the equation of the form " $y=m x+b$ ", where $y$ is the absorbance value at $595 \mathrm{~nm}, \mathrm{~m}$ is the slope of the curve, $\mathrm{x}$ is the protein concentration and $\mathrm{b}$ is the $\mathrm{y}$-intercept, was obtained. Finally, the extract protein concentration was determined by adding $7 \mu \mathrm{L}$ of the lysate to $793 \mu \mathrm{L}$ TBS buffer and $200 \mu \mathrm{L}$ Bradford Reagent. The slope and intercept from the generated standard curve equation was then used to calculate the concentration of the whole cell lysate sample based on the measured absorbance.

Below is a sample calculation for a representative Bradford Assay result (see Table 4 for individual absorbance values and standard curve plot)

$$
\begin{gathered}
\text { Control Sample: } \quad \mathrm{x}=\frac{(0.083+0.092) / 2}{3.95 \times 10^{-3}}=22.15 \mu \mathrm{g} / \mathrm{mL} \\
\text { UV Sample: } \quad \mathrm{x}=\frac{(0.135+0.122) / 2}{3.95 \times 10^{-3}}=32.52 \mu \mathrm{g} / \mathrm{mL} \\
\text { UV + Milk Phospholipids Sample: } \quad \mathrm{x}=\frac{(0.063+0.058) / 2}{3.95 \times 10^{-3}}=15.31 \mu \mathrm{g} / \mathrm{mL} \\
\text { Milk Phospholipids Sample: } \quad \mathrm{x}=\frac{(0.047+0.037) / 2}{3.95 \times 10^{-3}}=10.63 \mu \mathrm{g} / \mathrm{mL}
\end{gathered}
$$


The values above pertain to the concentration in the cuvette and the dilution factor had to be taken into account to obtain the total protein concentration of the whole cell lysate stock solution:

\author{
Control Sample: $\quad$ stock concentration $=\frac{(22.15 \mu \mathrm{g} / \mathrm{mL})(1 \mathrm{~mL})}{0.03 .16} \mathbf{~ m g} / \mathbf{m L}$ \\ $0.007 \mathrm{~mL}$ \\ UV Sample: $\quad$ stock concentration $=\frac{(32.52 \mu \mathrm{g} / \mathrm{mL})(1 \mathrm{~mL})}{0.007 \mathrm{~mL}}=\mathbf{3 . 6 5} \mathbf{~ m g} / \mathbf{m L}$ \\ $0.007 \mathrm{~mL}$ \\ UV + Milk Phospholipids Sample: $\quad$ stock concentration $=\quad(15.31 \mu \mathrm{g} / \mathrm{mL})(1 \mathrm{~mL})=\mathbf{2 . 1 8} \mathbf{~ m g} / \mathbf{m L}$ \\ $0.007 \mathrm{~mL}$
Milk Phospholipids Sample: $\quad$ stock concentration $=\underline{(10.63 \mu \mathrm{g} / \mathrm{mL})(1 \mathrm{~mL})}=\mathbf{1 . 5 2} \mathbf{~ m g} / \mathbf{m L}$ $0.007 \mathrm{~mL}$

\title{
3.5.3. Sodium Dodecyl Sulfate-Polyacrylamide Gel Electrophoresis
}

An established method often used to distinguish proteins of different molecular weights is sodium dodecyl sulfate-polyacrylamide gel electrophoresis (SDS-PAGE). In this technique, proteins are driven by an applied current through the acrylamide gel matrix, but in contrast to agarose gel electrophoresis, which separate molecules according to size and shape, and PAGE, which separate based on size, shape and charge density, SDS-PAGE separates solely based on the molecular mass of the molecules. This is made possible by SDS denaturation and coating of the proteins, which eliminate all inherent charges and impart an overall negative charge, and which reduce any higher structures into a rod-like protein molecule. The acrylamide pore sizes are important in determining the size of proteins that would be allowed to pass through its molecular sieve network of cross-linked polyacrylamide. Larger molecular weight proteins migrate slower due to their higher tendency to get entangled in the gel matrix and the proteins of interest are identified based on their mass as manifested by the distance of their migration relative to proteins of known size in the protein standard ladder.

The SDS-PAGE gel used was a commercial pre-cast gel, NuPAGE ${ }^{\circledR}$ Novex $4-12 \%$ Bis-Tris pre-cast gel (Invitrogen, Carlsbad, CA), and the only thing that needed to be prepared was the whole cell lysate sample mixture with the loading buffer according to volumes described in Table 3 . The mixture was then heated in a $70^{\circ} \mathrm{C}$ water bath for 10 minutes. 
Table 3. NuPAGE ${ }^{\circledR}$ Novex Bis-Tris Mini Gel sample preparation guide.

\begin{tabular}{|c|c|}
\hline Reagent & Reduced Sample Volume $(\boldsymbol{\mu L})^{*}$ \\
\hline Sample & 10 \\
\hline NuPAGE $^{\circledR}$ LDS Sample Buffer (4X) & 2.5 \\
\hline NuPAGE $^{\Theta}$ Reducing Agent (10X) & 1 \\
\hline Distilled $\mathrm{H}_{2} \mathrm{O}$ & 6.5 \\
\hline TOTAL VOLUME & 20 \\
\hline
\end{tabular}

* Volumes can be adjusted according to the desired loading volumes.

Whether using a hand-cast or pre-cast gel, the following gel electrophoresis procedures were performed. The white tape was ripped off the bottom and the cassettes were inserted into the Criterion Cell tank (Bio-Rad Laboratories, Hercules, CA) or XCell SureLock ${ }^{\mathrm{TM}}$ Mini-Cell Electrophoresis System (Invitrogen, Carlsbad, CA). The tank was then filled with running buffer (see Appendix for recipe) up to the fill line. Up to $40 \mu \mathrm{g}$ of samples and $10 \mu \mathrm{L}$ of the protein standard were then pipetted into the wells and the cell tank was covered with the matching lid. The cables were then plugged-in (by matching the colors) and the gel electrophoresis apparatus was run at $200 \mathrm{~V}$ for 40-60 minutes until the dye front reached the bottom of the gel. To check if the apparatus was running properly, the bottom of the gel was checked for rising bubbles as soon as the run button was hit. After running the gel, the apparatus was disconnected and disassembled, the gel was removed and the cassette was cracked open by pushing the cassette reservoir down the protruding end of the tank lid or using the spatula that came with the Mini-Cell apparatus. The gel was then carefully loosened from the cassette and placed in distilled water. One of the gels was stained with Coomassie Blue and the other gel was set aside while immersed in distilled water for the immunoblottong procedure.

\subsubsection{Coomassie Blue Staining}

The Coomassie Brilliant Blue R-250 dye (see Appendix for recipe) was prepared by pouring the solution in a plastic container for staining of the gels. The gel was then incubated in the solution at room temperature on an orbital shaker for at least an hour to overnight. Then, gels were destained by incubating in destaining solution (see Appendix for recipe) until the protein bands were distinct and the background staining was cleared. Stained gels were then visualized and captured using the Transilluminator light source of the ChemiDoc ${ }^{\mathrm{TM}} \mathrm{XRS}+\mathrm{System}$. Gels were stored indefinitely after visualization by incubating in the storage solution (see Appendix for recipe). 


\subsubsection{Immunoblotting}

SDS-PAGE alone is efficient in separating proteins based on molecular size but may be insufficient in determining the presence or quantity of a specific protein since a single band in the gel may contain up to 10 different proteins (Moebius et al. 2005). For immunoblotting, the gel region containing the proteins of interest were cut off and used for blotting. Using a ruler, the dimensions of the cut gel were measured. Next, 8 sheets of Bio-Dot SF filter papers were cut to about the same size as the measured dimensions. Upon setting up, four of the larger sheets were to go under the membrane and 4 of the smaller ones were to go on top of the membrane without touching the bottom filter papers. Two sheets of the larger filter papers were first soaked in Buffer A (see Appendix for recipe) and placed on the anode side of the TransBlot ${ }^{\circledR}$ SD semi-dry Electrophoretic Transfer Cell (Bio-Rad Laboratories, Hercules, CA). Bubbles were removed by rolling a test tube on top of the filter paper from edge to edge. Another 2 sheets of filter paper were then soaked in Buffer B (see Appendix for recipe) and placed on top of the papers soaked in Buffer A. Bubbles were again removed by rolling a test tube on top of the papers. Next, using forceps, the PVDF membrane was cut to the gel dimensions and soaked in methanol for 20 seconds, rinsed with water and placed on top of the filter paper soaked in Buffer B. A test tube was again rolled on top of the membrane to get rid of the bubbles. The gel was then immediately placed on top of the membrane and bubbles were removed by tapping them towards the edge of the gel. Four sheets of filter paper were then soaked in Buffer C individually and each was placed on top of the gel one by one, removing bubbles in between each step. The lid cover was then

placed to close the apparatus and the transblot set-up was run for a total transfer time of up to 30 minutes at $200 \mathrm{~V}, 4$ W, $400 \mathrm{~mA}$.

Once the transblot apparatus has finished running or when the prestained marker has been transferred sufficiently to the membrane blot, the membrane was taken out using forceps and the membrane was cut at the bottom left corner to aid with the orientation. The molecular weight marker was also cut out from the membrane to prevent its interaction with the subsequent staining reactions. When blocking step was included in the protocol, the membrane was incubated in the blocking buffer (see Appendix for recipe) for 1 hour on an orbital shaker at room temperature. Next, the membranes were washed 3 times with TBS-Tween for 5 minutes each with agitation. Several p21antibodies from different manufacturers were tried before finally settling with GeneTex anti-human rabbit p21 
antibody (GeneTex, Irvine, CA). This was prepared by diluting up to $5 \mu \mathrm{g} / \mathrm{mL}$ solution with TBS-T. The membrane was then probed for the protein of interest by incubating in the primary antibody solution overnight at $4^{\circ} \mathrm{C}$. On the next day, the membrane was incubated at room temperature for an additional hour at room temperature on an orbital shaker. The membrane was then washed three times with TBS-T, each for 5 min followed by incubation of the membrane with $40 \mu \mathrm{L} / 5 \mathrm{~mL}$ horseradish peroxidase-conjugated anti-rabbit secondary antibody (Code: 111-035-003, Jackson ImmunoResearch, West Grove, PA) for 1 hour with shaking at room temperature. The membrane was once again, washed three times with TBS-T for 5 minutes each with agitation. Signal development was then performed via chemiluminescent detection or chromogenic detection. For chemiluminescent detection, SuperSignal West Pico HRP substrate (Pierce, Rockford, IL) was prepared according to manufacturer instructions by mixing 1:1 ratio of the hydrogen peroxide solution and the luminal enhancer solution. The membrane was positioned in the center of the ChemiDoc $^{\mathrm{TM}} \mathrm{XRS}+$ System chamber and about $500 \mu \mathrm{L}$ of the solution was pipetted on top of the membrane, just enough to cover the entire surface. The chemi hi-sensitivity application was chosen from the Quantity One ${ }^{\circledR}$ program and the membrane was manually exposed for 1000 seconds and image acquisition was set to 60 images to acquire an image at about every 13 second-interval. For chromogenic visualization on the other hand, $12 \mathrm{mg}$ of diaminobenzidine (DAB) powder was weighed and added to $20 \mathrm{~mL}$ of TBS and $25 \mu \mathrm{L} \mathrm{H}_{2} \mathrm{O}_{2}$. The membrane was incubated until satisfactory color development was achieved about 10-30 minutes. The reaction was then stopped by adding tap water to rinse the membrane. Lastly, the membrane was air-dried and stored between two filter papers away from light. 


\section{Chapter IV. Results and Discussion}

Stress induced by environmental agents may cause carcinogenesis through different mechanisms such as alteration of genes, induction of reactive chemical species that trigger oxidative stress or by inducing damage to DNA. UVB makes up 1-10\% of solar radiation reaching the earth surface and is mostly absorbed in the epidermal region. The induction of mammalian UV response is mediated by several transcription factors including AP-1, NF$\kappa \mathrm{B}$ and $\mathrm{p} 53$. However, $\mathrm{p} 53$ is the only factor that is directly induced by photodamaged DNA, while the other two are induced in response to signal transduction events that occur on the cell surface remotely from the DNA damage site. Increased p53 levels are believed to induce cell cycle arrest, NER, and apoptosis. Upregulation of both p53 and p21 expression have been used in a number of studies as key markers for better estimates of exposure to noxious agents and the extent of DNA damage incurred by the cell or tissue. In addition, this permits a better understanding of the intermediate steps involved in the pathway between exposure to stress to the development of disease. To this end, p21 expression and other DNA damage markers in normal controls, milk treated and UV-irradiated skin tissue were evaluated in order to gauge the effects of milk phospholipids against UV-induced DNA damage.

\subsection{Fluorescence and Immunofluorescence Image Analysis}

The pilot study conducted, which involved counting of \% DNA fragments from 12 control and UV samples revealed a standard error of 6.8241 . When this error was used for sample size estimation, the ideal sample size obtained to acquire an experiment with $80 \%$ power was 13 (Figure 25). The power was set high enough to allow reasonable departures from the null hypothesis to be detected.

The epidermal thickness measurements revealed that on average, the thickness of the control samples is significantly lower than the thickness of any of the other treatment groups (p-value $=0.000$ ) (Figure27). The average thickness of the milk samples was significantly lower than UV + Milk Phospholipid samples (p-value $=0.0486)$ but was not significant when compared to UV (p-value $=0.9767$ ), and no significant difference was detected between the UV and UV + Milk Phospholipid groups (p-value $=0.1262)$. In particular, the highest average thickness 
measured in the UV + Milk Phospholipid samples may be correlated to the combined proliferative effects observed to be induced individually by UV and milk phospholipid treatment (both p-values $=0.000$ ) (Figure 28).

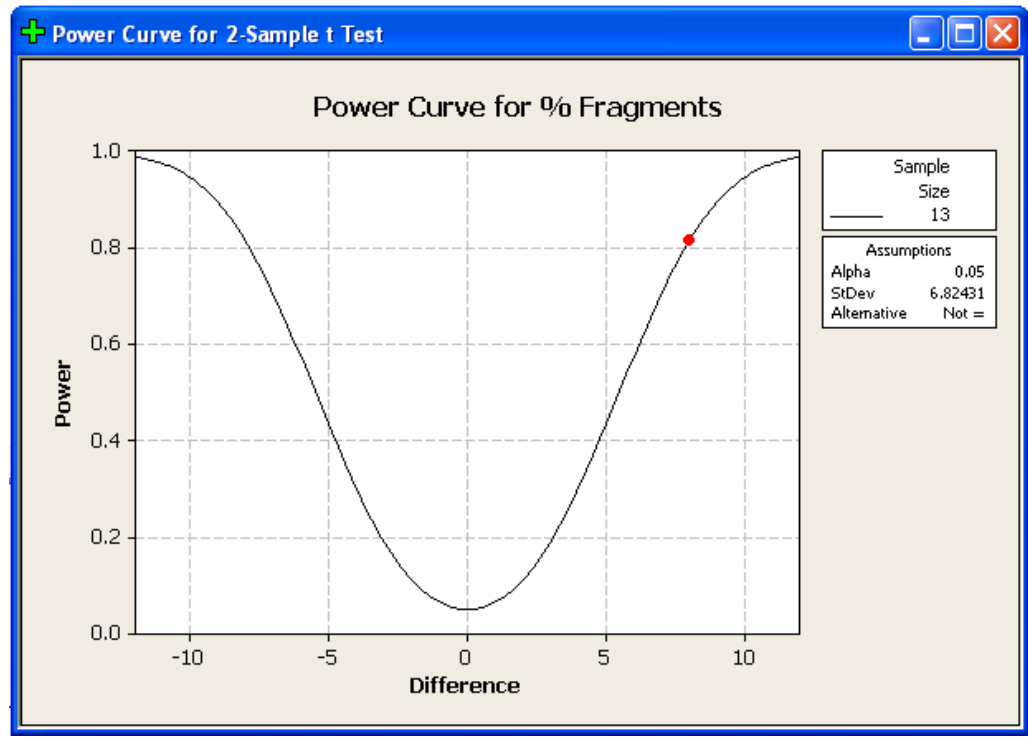

Figure 25. Power curve plot for sample size estimation and design of experiments with $\sim 80 \%$ power.

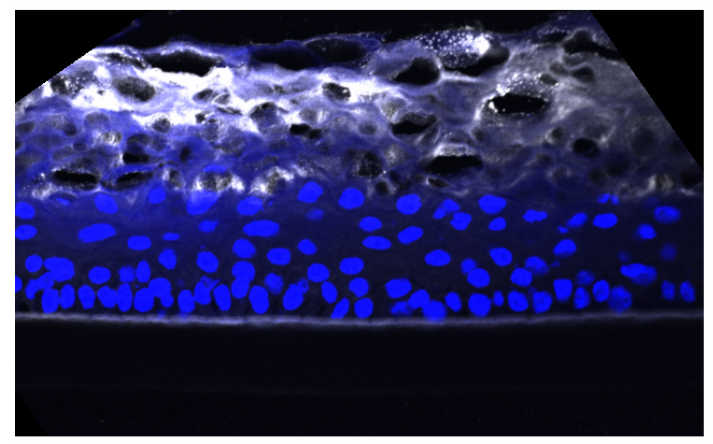

Control
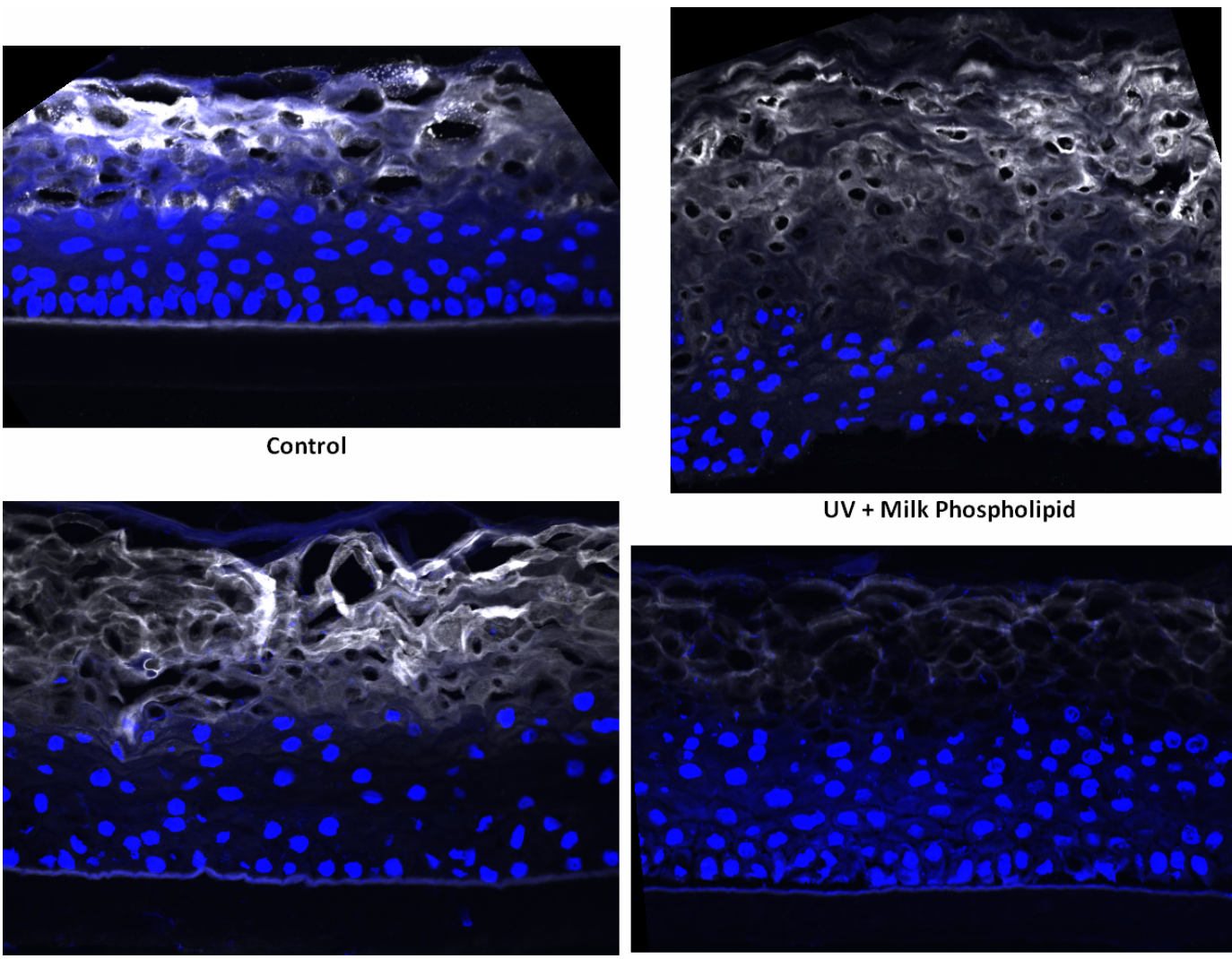

UV + Milk Phospholipid

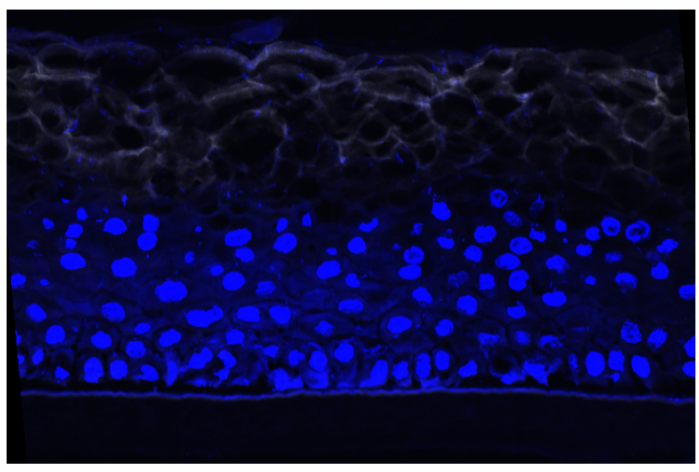

UV

Milk Phospholipid

Figure 26. Representative images for epidermal thickness comparison. 


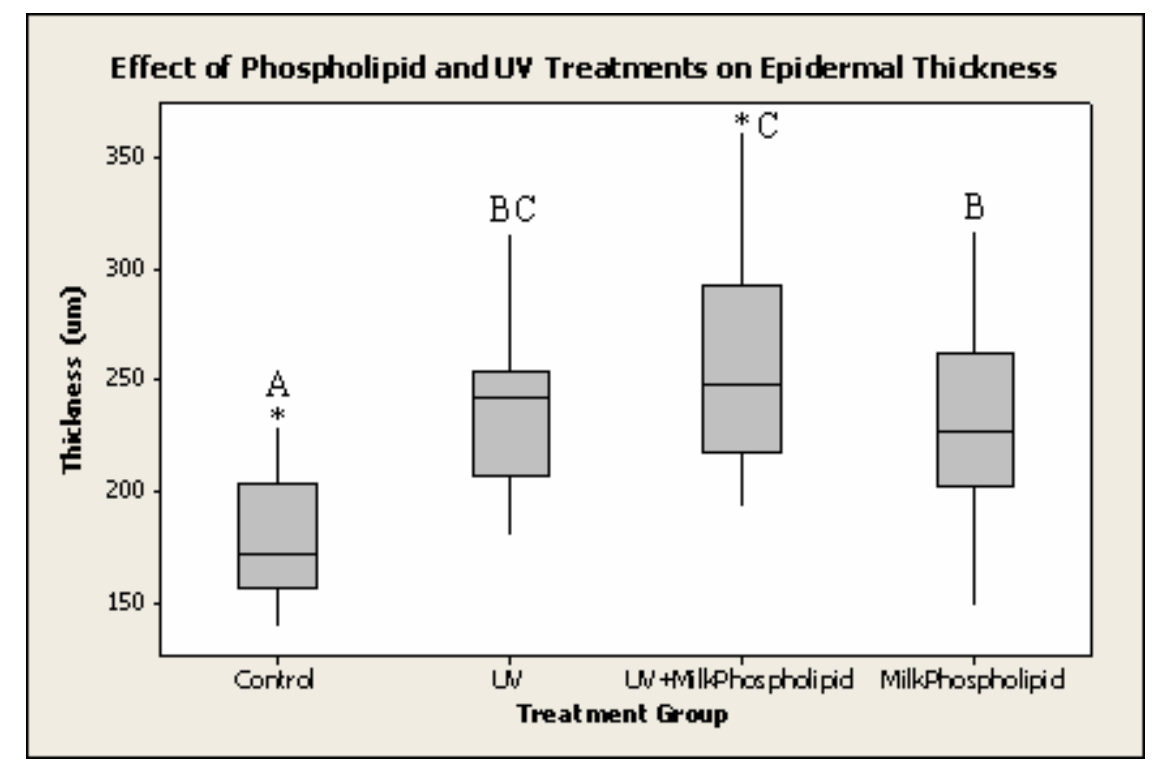

Figure 27. Boxplot of epidermal thickness measurements for each treatment group. Asterisks mark significant differences between sample groups and letters indicate their corresponding groupings after Tukey's pairwise comparison.

However, the apparent proliferative effects induced by phospholipid incubation and UV irradiation are two independent events as evidenced by the lack of interaction between the two treatment factors $(p$-value $=0.075)$ (Figure 29), and may thus involve different physiological pathways, which may in turn partly be accountable for their apparent cumulative effects on UV + Milk Phospholipid samples.

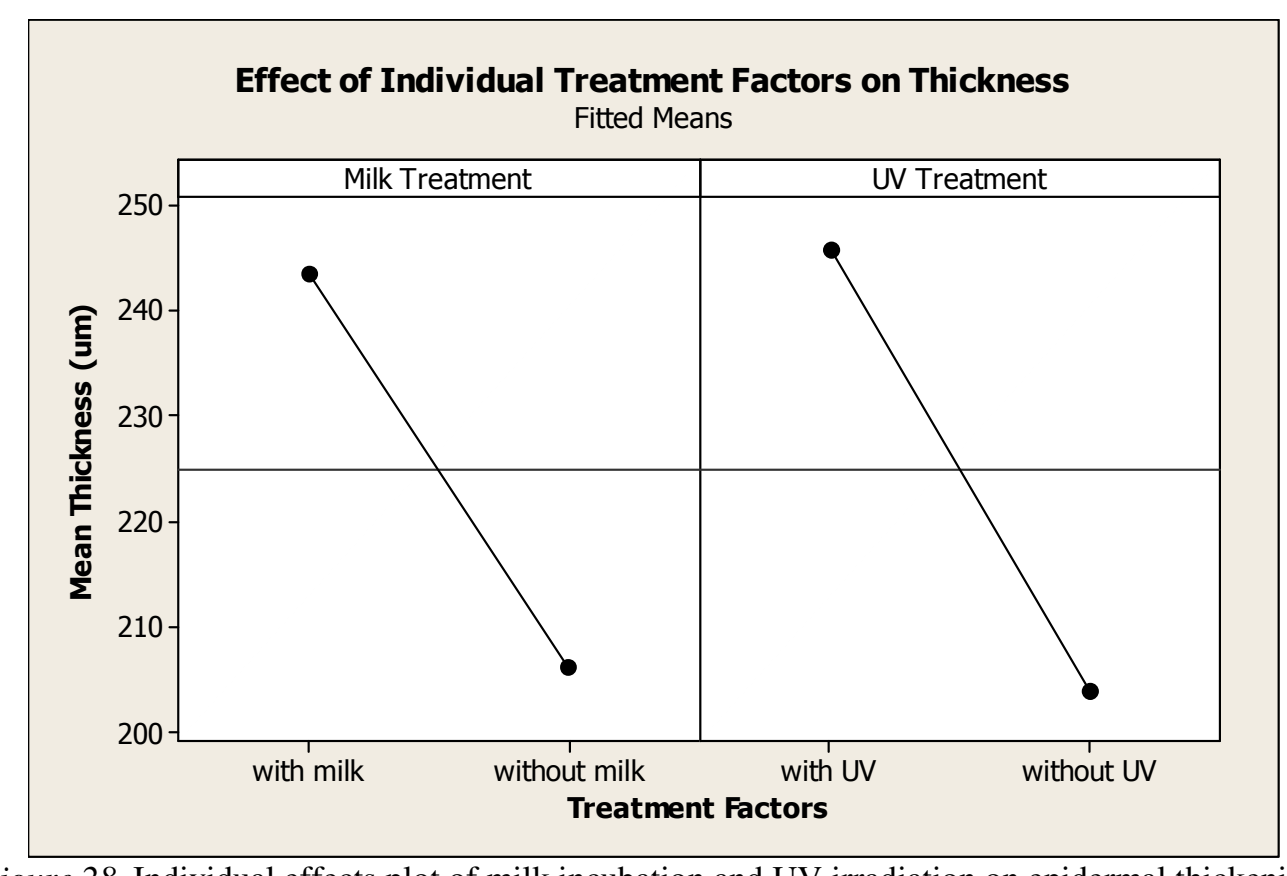

Figure 28. Individual effects plot of milk incubation and UV irradiation on epidermal thickening. 


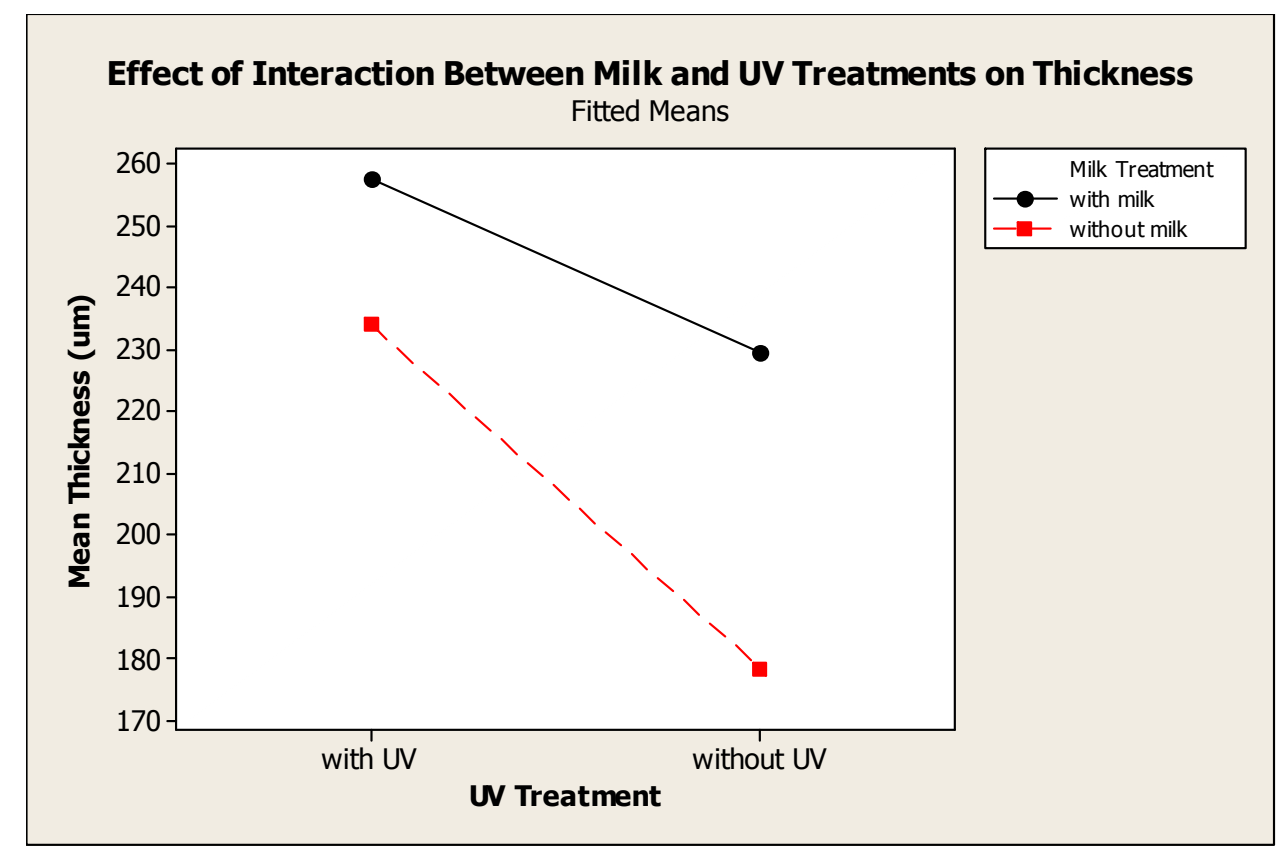

Figure 29. Interaction plot showing the lack of dependence between the two treatment factors in their effects on epidermal thickening.

The increase in thickness induced by UV is consistent with the hyperproliferative activity reportedly observed after UV irradiation, which is triggered as an immediate defense mechanism against possible further exposure. In contrast, the epidermal thickening effect of phospholipids on skin is unexpected, although it is known that phospholipid components such as posphatidylserine accelerates the recovery of damaged lipid barrier by increasing ceramide synthesis (mainly found in the stratum corneum) and blocks collagen reduction by inhibiting MMP-1 expession after UV irradiation. Perhaps, phospholipids are capable of promoting increased cell renewal even in the absence of stress which may act as a preventive mechanism. Another possible explanation for this occurence is that the unusually high concentration of the phospholipids used may be triggering a response pathway that leads to hyperplasia since slight changes in the concentration of several lipid moieties has been reported to induce contrasting effects on cell growth (Zhang et al. 1991, Ghosh et al. 1990).

The fluorescence of Hoechst dye is highly dependent on the DNA conformation and chromatin state in cells (Invitrogen, 2005). For this reason, they are very useful in distinguishing different degrees of nuclear damage. Some morphological features that are easily identified with Hoechst staining include nuclear condensation (Figure 30A), DNA fragmentation (Figure 30B), plasma membrane shrinkage and membrane blebbing. Results from DNA fragmentation analysis shows that the samples treated with UV alone sustained a significantly higher degree of DNA 
fragmentation compared to all other treatment groups (p-value $=0.000-0.005)$ (Figure 31$)$. Furthermore, the effect of UV irradiation on DNA fragmentation is dependent on milk phospholipid incubation of the samples (Figure 32), where phospholipid incubation prior to irradiation seemed to reduce the number of fragmented DNA in UV-exposed cells $(\mathrm{p}$-value $=0.000)$. The explanation behind the apparent protective effect of phospholipid against DNA damage in this case is unclear, but may be a result of the milk phopholipids' possible participation in damage response or repair through the phospholipid's cell signaling activities or perhaps, due to a possible role in enhancing the barrier properties of the skin against radiation.

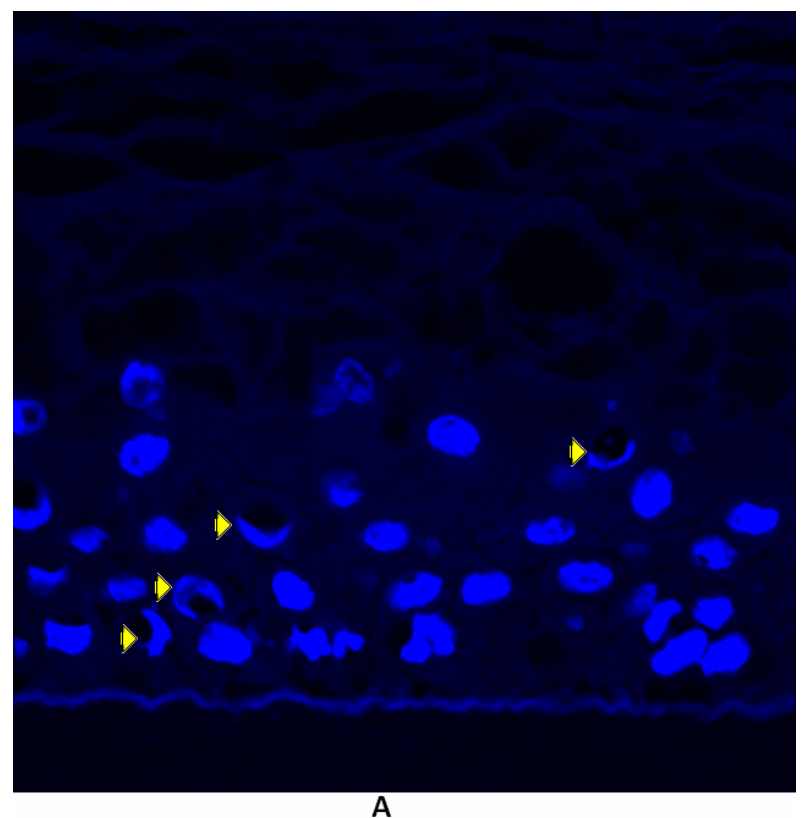

A

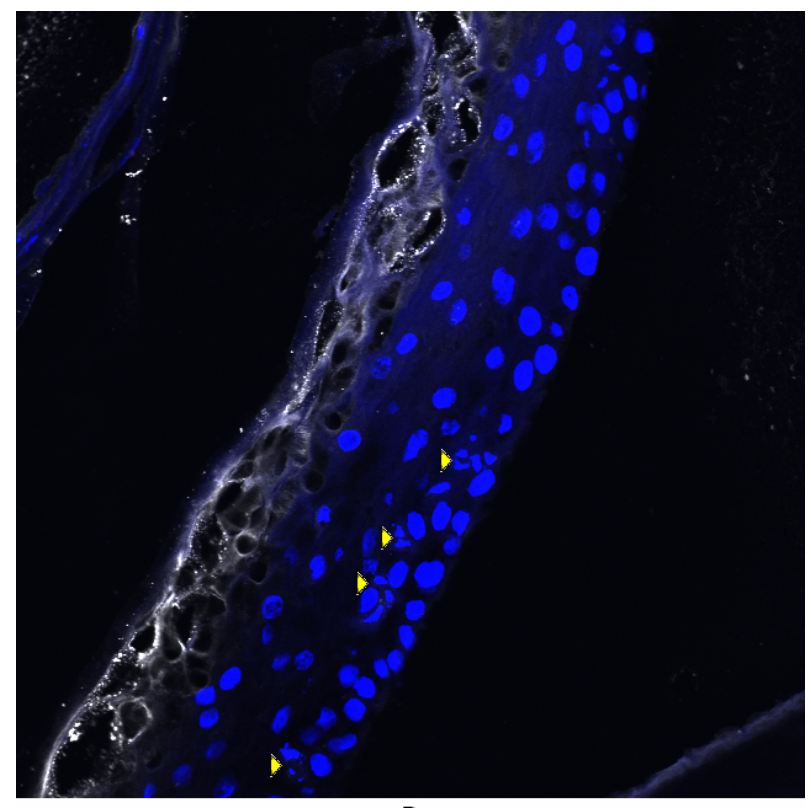

B

Figure 30. Representative images of cells exhibiting nuclear condensation (A) and DNA fragmentation (B) as indicated by the yellow arrowheads. 


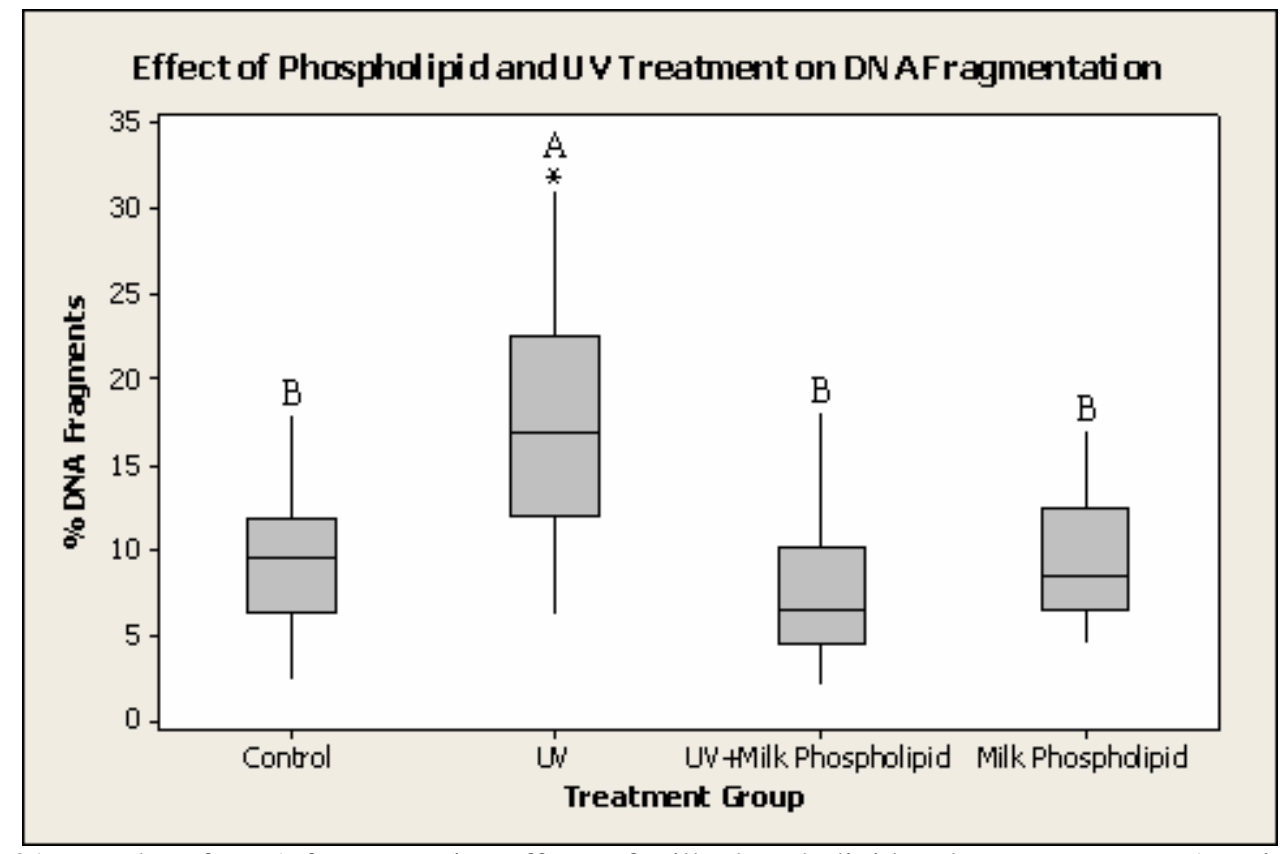

Figure 31. Boxplot of DNA fragmentation effects of milk phospholipid and UV treatments. Asterisk marks significantly different sample group and letters indicate their corresponding groupings after Tukey's pairwise comparison.

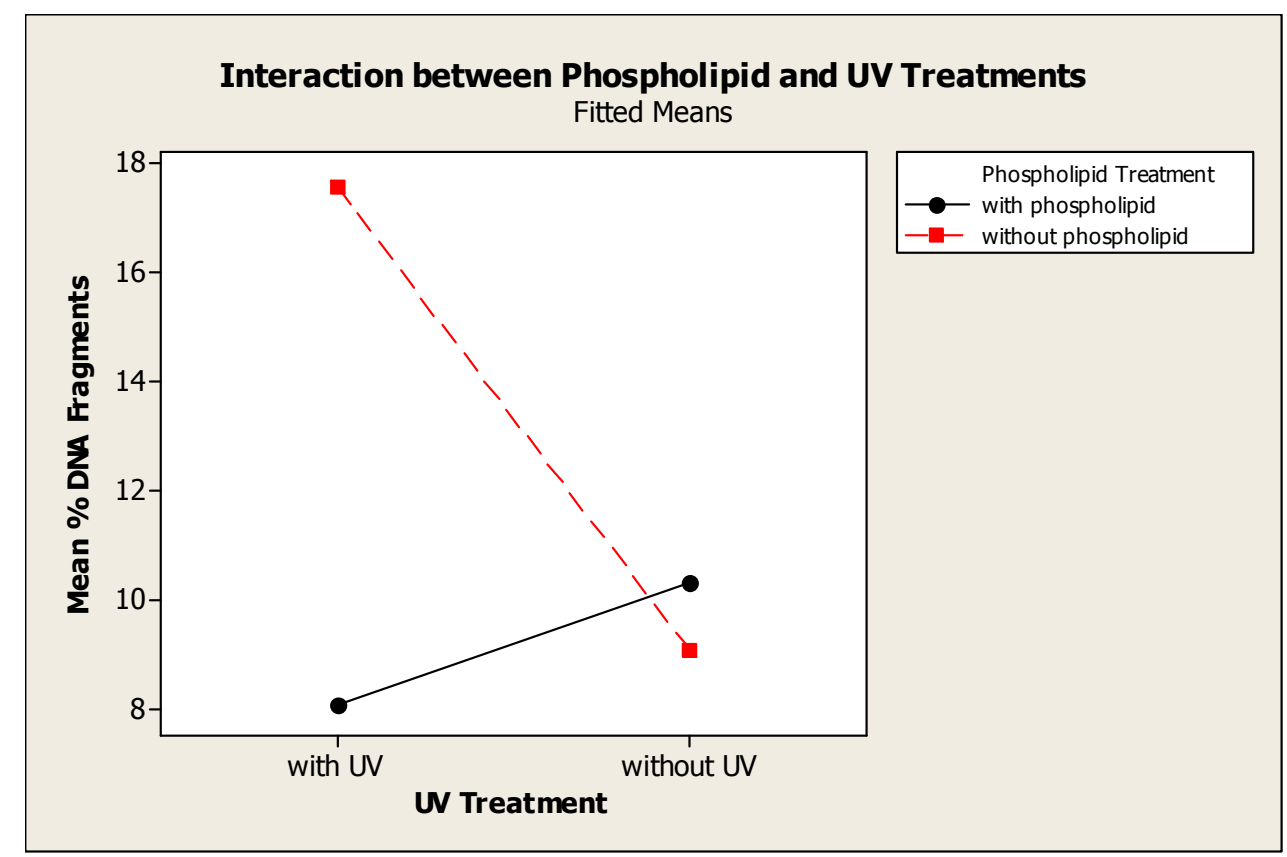

Figure 32. Interaction plot showing the dependence of UV radiation's DNA fragmentation effect on prior milk phospholipid incubation.

The visible light absorption maximum of Alexa Fluor ${ }^{\circledR} 647$ is $649 \mathrm{~nm}$ and the emission maximum occurs at $666 \mathrm{~nm}$. The use of the "AND" function to merge the Hoechst and p21-stained image channels proved to be very 
helpful in eliminating most of the background staining that were otherwise failed to be reduced sufficiently by the blocking step. In effect, it allowed easy identification of p21 staining that specifically occurs only inside the nucleus of cells. These cells are of particular interest because p21 proteins upregulated by p53 localizes within the nucleus where their function is to bind to and inhibit nuclear proteins necessary for cell cycle progression. At the same time, the stark contrast between the bright green, red and black colors in the resulting images allows easy visualization of the amount of signal present in each nucleus, which when developed further may be useful in conducting a more quantitative analysis of p21 expression. This technique however, may not account for false positives that may be generated by signal crossover or bleed-through, especially given the high laser power used to detect signals in the Alexa ${ }^{\circledR}$ Fluor 647 channel. Bleed-through can be controlled by reducing the laser power, photomultiplier voltage and gain and by modifying the detector entrance slit bandwidths or the emission filter spectral profile. Another technique to minimize bleed-through is to activate the "Sequential Scan" checkbox to acquire images from different channels in sequential order rather than simultaneously, and to separate the channels with similar spectra into different "Groups" from the menu toolbar.

Statistical analysis of p21 immunofluorescence staining data shows that the UV samples had significantly higher percentage of p21-positive cells $(0.0004-0.0251)$ compared to any other treatment group, whereas no siginificant difference in the amount of \% p21-positive cells exists between any of the three remaining groups (pvalue $=0.5109-0.9244)($ Figure 33). The relatively low p21 expression exhibited by the UV + Milk Phospholipid samples despite exposure to UV implies the protective effects of milk phospholipid incubation against radiationinduced DNA damage. This dependence of the UV treatment's p21 upregulation effect on milk incubation is illustrated in their interaction plot (Figure 34), which shows that the difference in p21 expression between exposed and non-exposed tissues is statistically significant when they are not incubated with phospholipids while the effect of exposure is reduced when samples are incubated in phospholipids. 


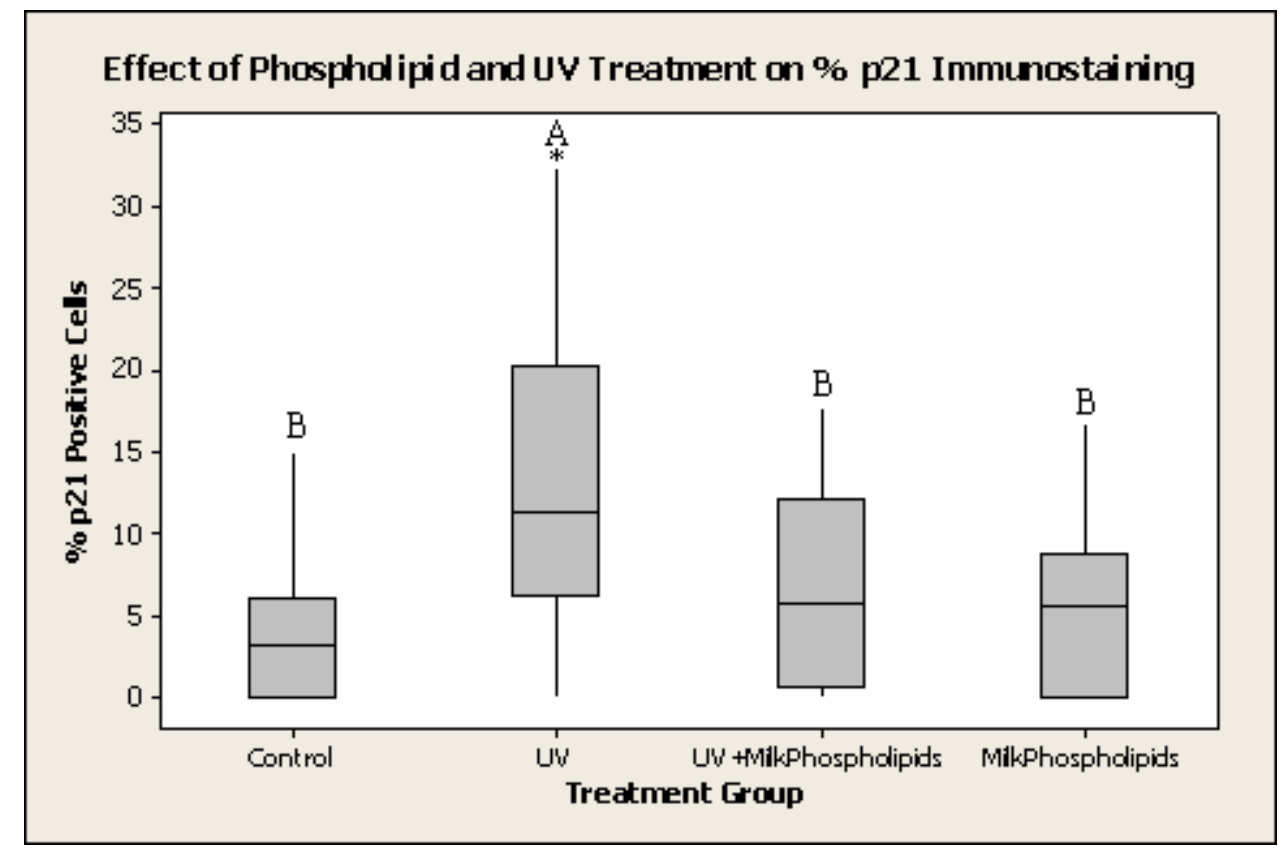

Figure 33. Effect of phospholipid and UV treatment on the amount of p21. Asterisk marks significantly different sample group and letters indicate their corresponding groupings after Tukey's pairwise comparison.

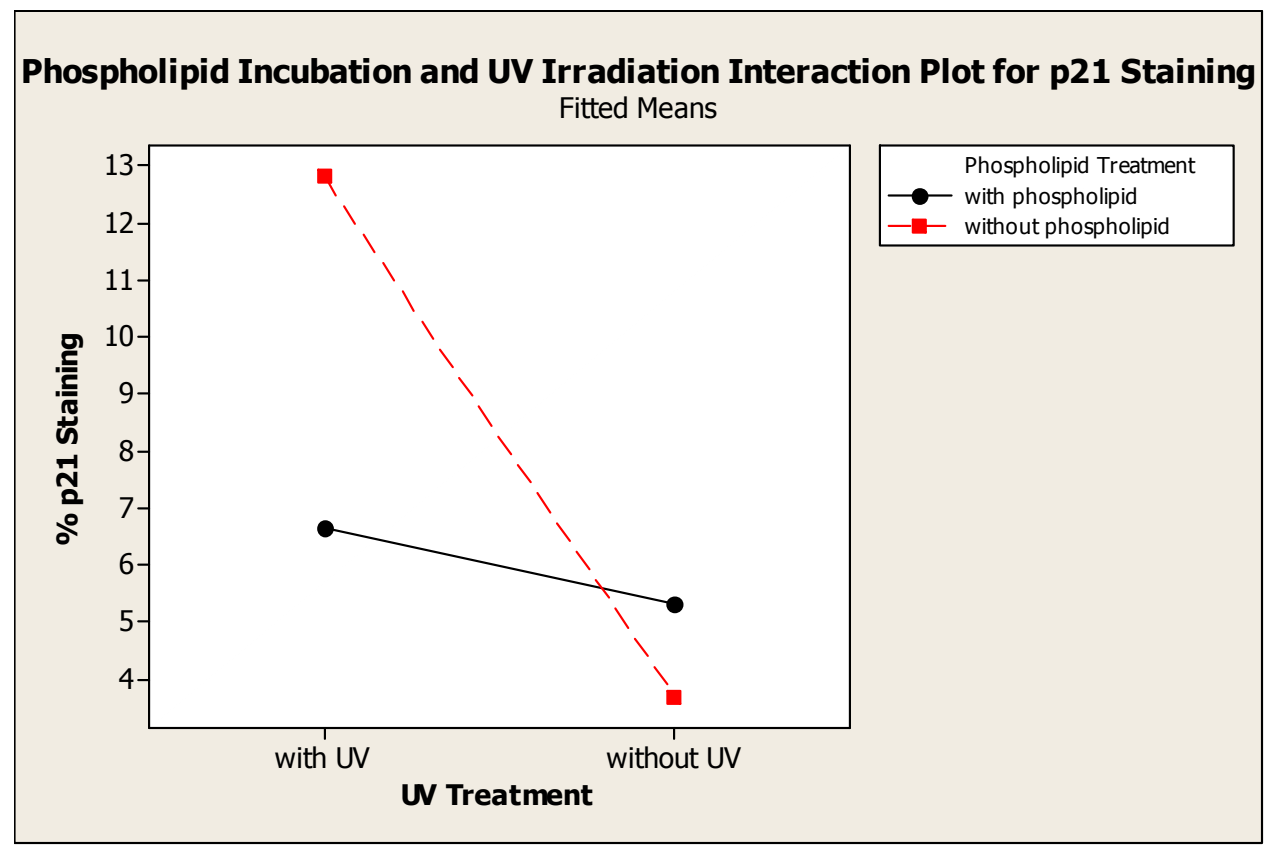

Figure 34. Interaction between Phospholipid and UV treatments showing the dependence of UV treatment's p21 upregulation effects on milk phospholipid incubation. 


\subsection{Western Blot Analysis}

The Western blot technique was beset with problems including the large amount of proteins required ( 50 $100 \mathrm{mg}$ ) in order to detect a signal, protein loss and degradation during the extraction procedure, difficulties in achieving effective separation and retaining small proteins during electrophoresis and efficient transfer of the total proteins with a wide range of molecular weights. Extraction from intact epidermal skin tissue in particular, is considered relatively difficult since the skin is naturally designed to be very tough and can withstand a reasonable amount of mechanical pressure, friction and shear force before completely disintegrating. It was thus necessary to use harsher chemicals and mechanical force to homogenize the tissue, free individual cells by successfully breaking down extracellular matrix and junctions that holds the skin cells together and ultimately, lyse the plasma and nuclear membranes to release $\mathrm{p} 21$ proteins in intact and undenatured form from the cytoplasm and nucleus. The skin also contains high levels of proteases and several layers of cells (i.e. stratum corneum) which do not contribute to the total amount of nuclear proteins of interest, thereby decreasing the purity and ratio of nuclear to total proteins in the resulting lysate.

Due to the necessity of employing harsh lysis techniques to break down the skin structure and to obtain a high quantity of the low abundance p21 protein for Western blot, the extraction method used for this study was adapted from protocols specific for filamentous fungi (Pasquali et al. 2010) and plant tissues (Zheng et al. 2007), which are often problematic samples due to their low protein content and the abundance of interfering substances. Protein extraction protocols for fungi and bacteria are especially harsh due to the need for shearing their stable cell walls that are stronger than plasma membranes, while plants require methods that can dirupt their rigid cellulose cell wall. Breakdown of solid tissue and membrane lysis aims to disrupt cell interconnections and weaken the cell membrane in order to release proteins. SDS is an ionic detergent with a negatively charged head group and was found to be most effective at producing the highest protein yield by virtue of its strong denaturant characteristics. It denatures and solubilizes by hydrophobic interactions with protein chains to destroy ionic interactions between proteins and impart a negative charge to them that causes electrostatic repulsion between the chains (Speers and $\mathrm{Wu}$, 2007). 
Typical protein concentrations of whole cell lysates obtained using the 2\% SDS extraction protocol with $\mathrm{TCA}$ /acetone precipitation ranged from $1 \mathrm{mg} / \mathrm{mL}$ to $4 \mathrm{mg} / \mathrm{mL}$ (Table 4), which is within the optimal concentration range in typical protein extraction procedures. The standard curve generated from the Bradford Assay was typically linear as expected (Figure 35). The Bradford Assay, unlike other protein determination assays (e.g. Lowry, HartreeLowry, Biuret and Bicinchoninic Assay), is not susceptible to most chemical interference usually present in lysates except high detergent concentrations. The effect of high detergent concentration is an increase in variation of the measured absorbance values between different proteins and thus, careful selection of a protein standard with highly similar absorbance values to the protein of interest is most suitable. Also, since different proteins and nucleic acids have widely varying absorbance properties, a whole cell lysate sample with possibly high mixture of UV-absorbing components and low protein purity may give considerable error to the absorbance measurements.

Table 4. Representative Bradford Assay absorbance values of BSA standards and tissue samples after 2\% SDS extraction procedure followed by TCA/acetone precipitation.

\begin{tabular}{|c|c|c|c|c|}
\hline Sample & $\begin{array}{c}\text { Absorbance (AU) } \\
\mathbf{1}^{\text {st }} \text { Reading }\end{array}$ & $\begin{array}{c}\text { Absorbance (AU) } \\
\mathbf{2}^{\text {nd }} \text { Reading }\end{array}$ & $\begin{array}{c}\text { Absorbance (AU) } \\
\text { Average }\end{array}$ & $\begin{array}{c}\text { Stock } \\
\text { concentration } \\
(\mathrm{mg} / \mathrm{mL})\end{array}$ \\
\hline BSA $(4 \mu \mathrm{g} / \mathrm{mL})$ & 0.108 & 0.111 & 0.110 & \multirow{5}{*}{$(-8)$} \\
\hline $\mathrm{BSA}(16 \mu \mathrm{g} / \mathrm{mL})$ & 0.208 & 0.208 & 0.208 & \\
\hline BSA $(25 \mu \mathrm{g} / \mathrm{mL})$ & 0.228 & 0.223 & 0.226 & \\
\hline BSA $(37 \mu \mathrm{g} / \mathrm{mL})$ & 0.246 & 0.243 & 0.245 & \\
\hline BSA $(44 \mu \mathrm{g} / \mathrm{mL})$ & 0.295 & 0.285 & 0.290 & \\
\hline Control & 0.092 & 0.083 & 0.088 & 3.16 \\
\hline UV & 0.135 & 0.122 & 0.129 & 3.65 \\
\hline UV + Milk Phospholipids & 0.063 & 0.058 & 0.061 & 2.18 \\
\hline Milk Phospholipids & 0.047 & 0.037 & 0.042 & 1.52 \\
\hline
\end{tabular}

Based on the absorbance readings, a high amount of protein was present in the UV lysate sample whereas very little amount was extracted from the milk phospholipids lysate. Different stock concentration trends observed in repeated experiments suggests that the total amount of proteins acquired from the lysate at each extraction is mostly a consequence of variations in the extraction technique than a measure of the actual protein quantity present in the tissue samples. Consequently, increasing the sample size (by doing repeat experiments) of our Western blot data for p21 expression would be helpful to ensure the reproducibility of the results we obtained. 


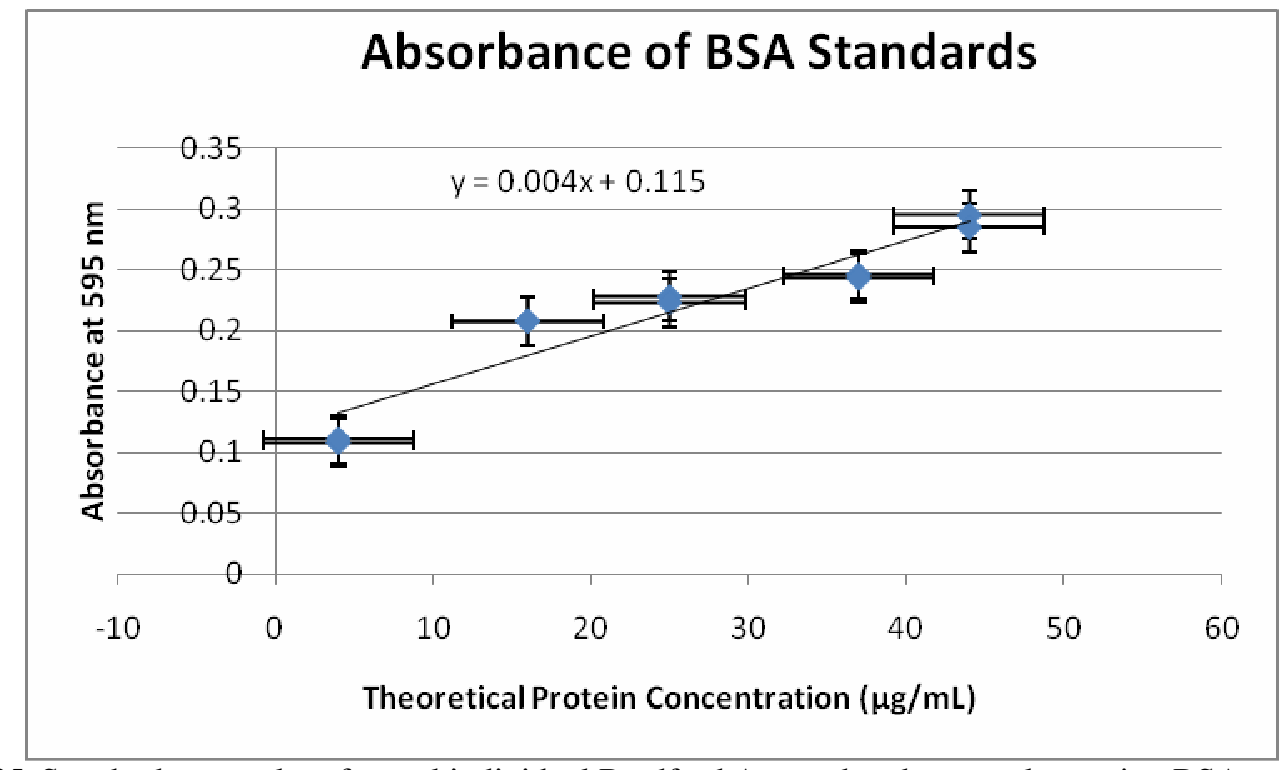

Figure 35. Standard curve plot of actual individual Bradford Assay absorbance values using BSA standards of increasing concentration.

The protein of interest is expected to be seen at $18 \mathrm{kDa}$ when run in a gel, but exists as a $21 \mathrm{kDa}$ protein in vivo. We used positive controls for $\mathrm{p} 21$ in our testing in order to circumvent variables with protein concentration and to verify the efficacy of parameters in our Western blot protocol. Specifically, C32 nuclear extracts, derived from amelanotic melanoma cells were used (Figure 36). Malignant melanomas are typically characterized by high levels of p53 and therefore, p21 expression (Kichina et al. 2003). The appearance of several bands in the positive controls may be a result of using polyclonal anitbodies, which attach to several regions (epitopes) supposedly in the antigen of interest, thereby generating several strong signals. In contrast monoclonal antibodies, which have more specificity often produce weaker signal intensity.

After much optimization of the Western blot protocol, moderate background was still incompletely eliminated but several protein bands were distinguishable. In particular, the putative p21 protein band appeared between molecular weights 15 and $20 \mathrm{kDa}$ in the positive control, Milk Phospholipids and UV sample lanes in the blot detected with chemiluminescent substrate (Figure 37B, Lanes 2, 4 \& 5). In contrast, only the UV sample lane contained the putative p21 protein band when chromogenic detection was used (Figure 37C \& D, Lane 2). 

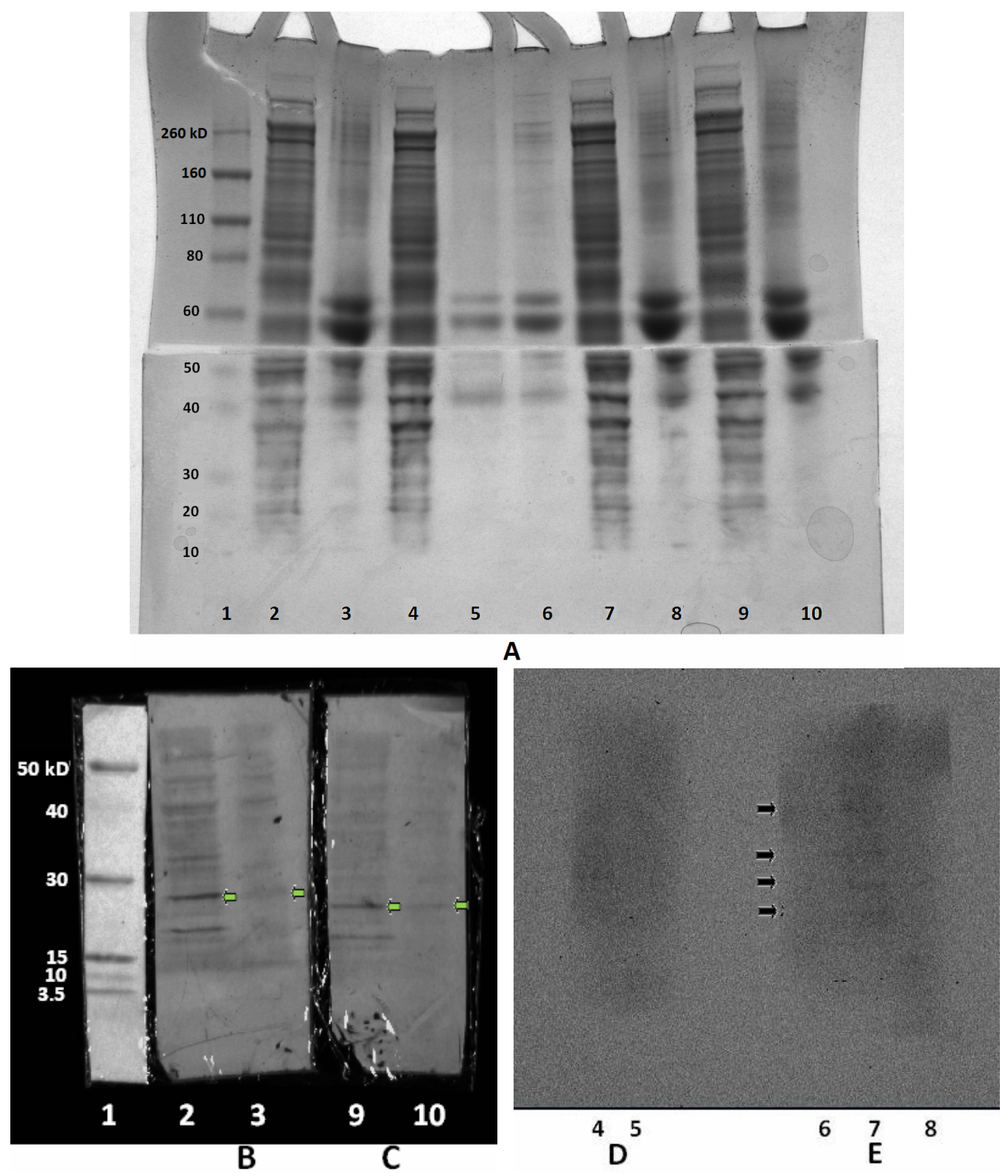

Figure 36. Western blot optimization of blocking step with positive control and UV samples. Samples loaded in the 4-12\% pre-cast SDS-PAGE gel $(A)$ include $37 \mu \mathrm{g} \mathrm{C} 32$ nuclear extract (lanes 2, 4, $7 \&$ 9), $43 \mu \mathrm{g} \mathrm{UV}$ (lanes 3, 5, 8 \&

10) and $38 \mu \mathrm{g}$ Control (lane 6 ). Note that there was a spill during the loading of lane 5 and variable protein concentrations were loaded since the run was performed only to verify if a signal can be detected. After 30 minutes protein transfer, all blots $(B, C, D \& E)$ were incubated directly with $5 \mu \mathrm{g} / \mathrm{mL}$ Genetex p21 primary antibody diluted in TBS-T without a prior blocking step. Blots B and D were then incubated in $8 \mu \mathrm{g} / \mathrm{mL}$ Jackson Immunoresearch

HRP-conjugated secondary antibody diluted in Blotto A buffer, whereas blots $\mathrm{C}$ and $\mathrm{E}$ were incubated in same

concentration of secondary antibody diluted in TBS-T. Signals in blots B and C were detected using DAB

chromogenic substrate, while blots D and E were developed using SuperSignal West Pico chemiluminescent substrate. Green arrows point to putative p21 protein bands and black arrows point to faint extraneous bands in the positive control lane. Images B \& C were captured 1 day after color development. 

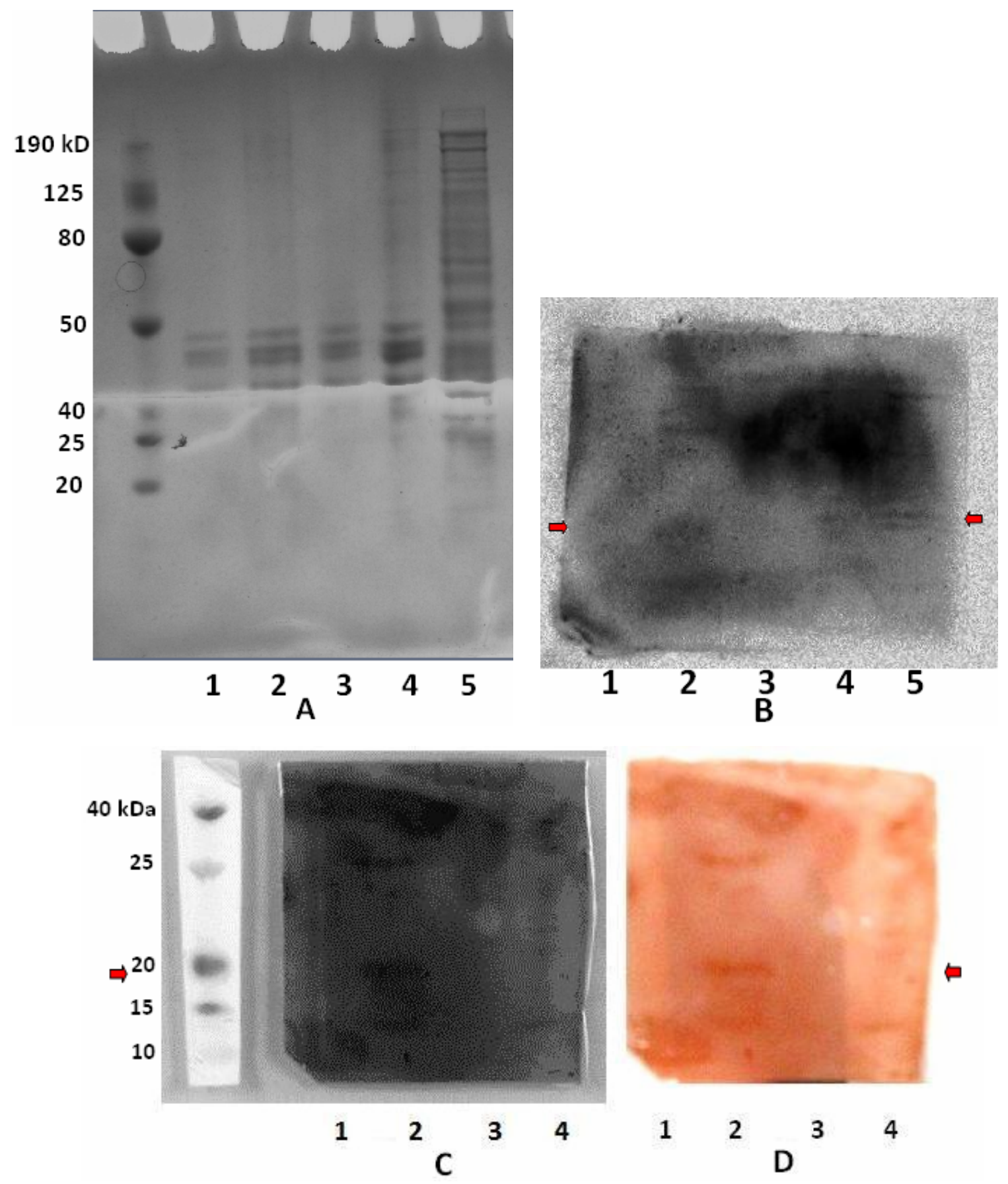

Figure 37. Western blot analysis of p21 expression in human skin tissue equivalents. A 4-12\% pre-cast SDS-PAGE gel (A) was loaded with $10 \mu \mathrm{L}$ ladder, $33 \mu \mathrm{g}$ each of Control (lane 1), UV (lane 2), UV + Milk Phospholipids (lane 3), Milk Phospholipids (lane 4) and C32 nuclear extract (lane 5) samples, as measured using Bradford Assay. Skin equivalent tissues were extracted using $2 \%$ SDS protocol with TCA/acetone precipitation. The molecular weight ladder used was HyperPage Prestained Protein Standard. Blots $(B, C \& D)$ were incubated with $5 \mu \mathrm{g} / \mathrm{mL}$ Genetex p21 primary antibody in TBS-T without a prior blocking step due to loss of signal with a blocking step. Blots were then probed with $8 \mu \mathrm{g} / \mathrm{mL}$ HRP-conjugated secondary antibody in Blotto A buffer. Signals in blot $B$ were detected with SuperSignal West Pico chemiluminescent substrate. $C$ and $D$ are images of the same blot developed by adding a DAB substrate to the membrane. Resulting bands were then imaged with ChemiDoc XRS+ under epi-illumination light source $(C)$ or captured with a Canon PowerShot A470 point-and-shoot camera $(D)$. 
ImageJ gel analysis tool was used to compare p21 protein band density of the skin equivalent tissues relative to the band density of the $\mathrm{C} 32$ nuclear extract positive control in the chemiluminsecent blot (Figure 38) but not in the chromogenic blot due to the lack of a positive control lane in the latter. Although it was intended to load equivalent amounts of protein in each lane based on computations from the Bradford Assay, it was evident that after running and staining the gel, the size and intensity of the bands varied between lanes. This may be due to several factors such as inaccuracies during the Bradford Assay procedure, incomplete resuspension of proteins with TBS buffer and poor pipetting techniques. Because of this, the density values of the other bands may not be directly comparable to each other.

ImageJ's gel analysis results for the gel's relative band densities were used to scale the values in the chemiluminescent blot image (Figure 39). This was based on the assumption that the proportional differences in the relative densities of the gel bands are identical to the amount of total proteins loaded in the gel (Miller, 2010). The Western blot relative density was then adjusted to the gel relative density by dividing the relative density values in the blot by the relative density values in the gel (Table 5).

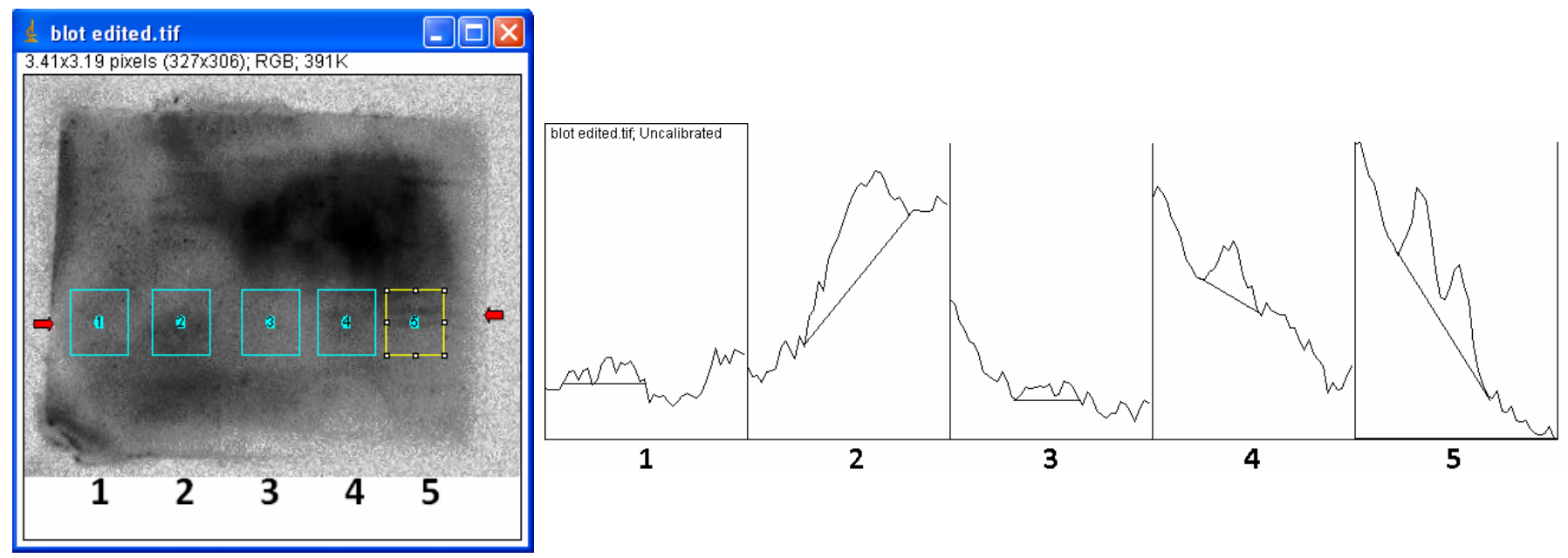

Figure 38. ImageJ gel analysis of chemiluminescent blot p21 bands. Rectangular regions of equal areas were plotted and the areas under the highest peaks to their baseline were measured. 


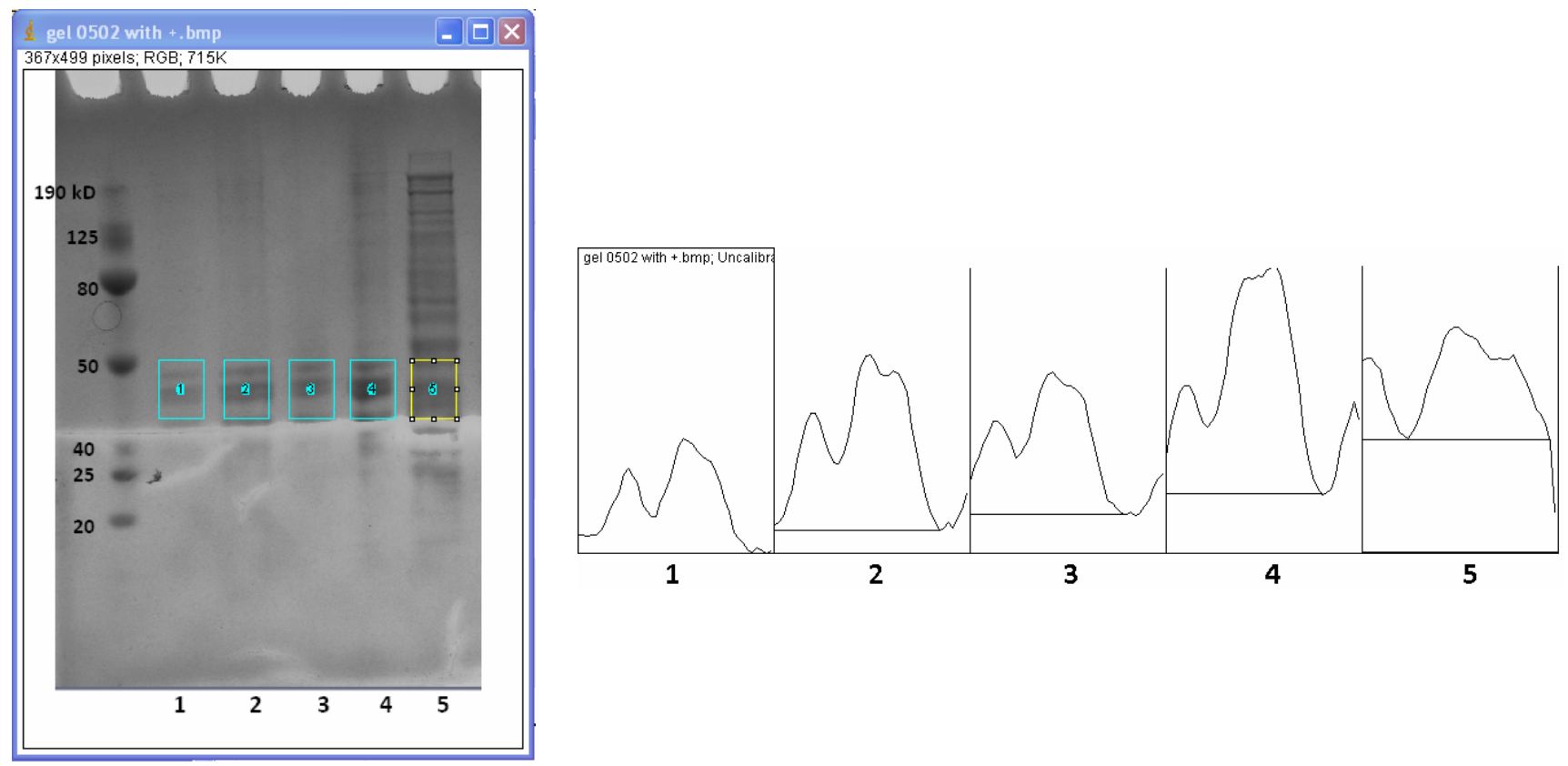

Figure 39. ImageJ gel analysis of Coomassie blue-stained gel protein bands. Rectangular regions of equal areas were plotted and the areas under the highest peaks to their baseline were measured.

Table 5. Relative band intensity analysis of the gel and chemiluminescent blot.

\begin{tabular}{|c|c|c|c|c|}
\hline Gel Lane & $\begin{array}{c}\text { Area under the } \\
\text { Curve }\end{array}$ & Percent & Relative Density & \\
\hline 1 & 7599.045 & 14.09 & 0.84 & \\
\hline 2 & 12165.75 & 22.55 & 1.34 & \\
\hline 3 & 9620.681 & 17.84 & 1.06 & \\
\hline 4 & 15454.27 & 28.65 & 1.70 & \\
\hline 5 & 9100.167 & 16.87 & 1.00 & \\
\hline Blot Lane & & & & Adjusted Density \\
\hline 1 & 572.163 & 5.91 & 0.18 & 0.21 \\
\hline 2 & 4269.501 & 44.08 & 1.32 & 0.99 \\
\hline 3 & 507.870 & 5.24 & 0.16 & 0.15 \\
\hline 4 & 1111.497 & 11.48 & 0.34 & 0.20 \\
\hline 5 & 3224.488 & 33.29 & 1.00 & 1.00 \\
\hline
\end{tabular}

Based on data from the adjusted relative density, p21 expression was highest for the UV sample among the four treatment groups and was comparable to levels seen in the positive control. In contrast, the sample treated with milk phospholipids prior to UV irradiation expressed low levels of p21. Likewise, the adjusted relative density values of the Control and Milk Phospholipid samples were low.

The levels of p21 expression observed in the Control samples may be consistent with what is expected to be seen at normal physiological levels. In normal cells, baseline p53 levels are low (Oren et al. 1981) and so is their transcriptional activity (Rogel et al. 1995). Upregulation of p21 protein therefore, provides a fairly defined 
assessment of p53 activity since the presence of activated p53 transcriptionally induces high levels of p21 production (Halaban et al. 1984; Kastan et al. 1992; el-Deiry et al. 1993; 1995). Based on the adjusted relative density values, it is possible that the Milk Phospholipid sample expression level observed in the blot would have been comparable to that seen in the Control sample if not for the uneven protein loading in the gel.

To further supplement our data, a negative control containing $1 \%$ milk phospholipid diluted in maintenance media was run along with C32 nuclear extract positive control and melanoma whole cell lysate samples (Figure 40). At the same time, a $0.5 \%$ casein blocking buffer was used to dilute the secondary antibody in the second half of the blot (Figure 40B, lanes 5, $6 \&$ 7). The p21 protein was undetectable in any of the lanes that used Blotto A as blocking buffer for the secondary antibody (Figure 40B, lanes $2,3 \& 4$ ). This is inconsistent with previous results (Figures $36 \& 37$ ) where the positive controls gave fairly strong signals. This may be partly due to gel distortion (Figure 40A, Lane 3) or the unusually high background that has also obscured protein standard lane visualization (Figure 40B, Lane 1). In turn, the latter may be caused by errors in weighing out the DAB substrate or failure to stop the color development reaction in time.

Likewise, no signal was detected in melanoma samples (Figure 40B, Lanes 4 \& 7). The mechanism behind this lack of p21 expression in the melanoma whole cell lysate is not clear, considering C32 extracts derived from amelanotic melanoma cell lines generate a fairly strong signal for the p21 protein. A likely explanation for this apparent discrepancy in the results may have to do with the low quantity of $\mathrm{p} 21$ proteins present in our melanoma samples compared with the loaded positive controls (Figure 40A, Lanes 3, 4, 6 \& 7). Although the loaded total protein concentrations were equalized based on their calculated Bradford Assay concentrations, the ratio of nuclear to total proteins in the whole cell lysates may have been considerably lower in melanoma samples than in nuclear extracts. Research has also shown that p21 induction in human melanoma cell lines is independent of wild-type p53 expression (Jiang et al. 1995; Vidal et al. 1995) and is often associated with melanoma differentiation, growth arrest and metastatic suppression (Jiang et al. 1995). This may imply that the melanoma whole cell lysate sample and C32 nuclear extract sample may be at different differentiation and/or metastatic states at the time of testing. Nonetheless, the casein-blocked blot as expected, reveals the presence of a faint $\mathrm{p} 21$ protein band on the positive control lane along with stronger staining extraneous bands indicated by the black arrows. 
A strong $25-30 \mathrm{kDa}$ band in the negative control lane was seen in the Coomassie-stained gel, which may pertain to a yet unidentified lipid molecule present in high amounts in the phosphlipid mixture. The exact components of the milk phospholipid complex mixture is difficult to identify mainly because the analysis techniques that have been used in various studies for analyzing the components are focused on identifying individual molecules that are present rather than their quantity, due to inherent difficulties in the extraction and analysis of these compounds (Ziesel et al. 1986). Sphingomyelin however, is easy to analyze in high amounts and milk is estimated to contain about $123 \mathrm{nmol} / \mathrm{mL}$ of these molecules, but it is not likely to produce the observed band since its molecular weight is about $731 \mathrm{Da}$.
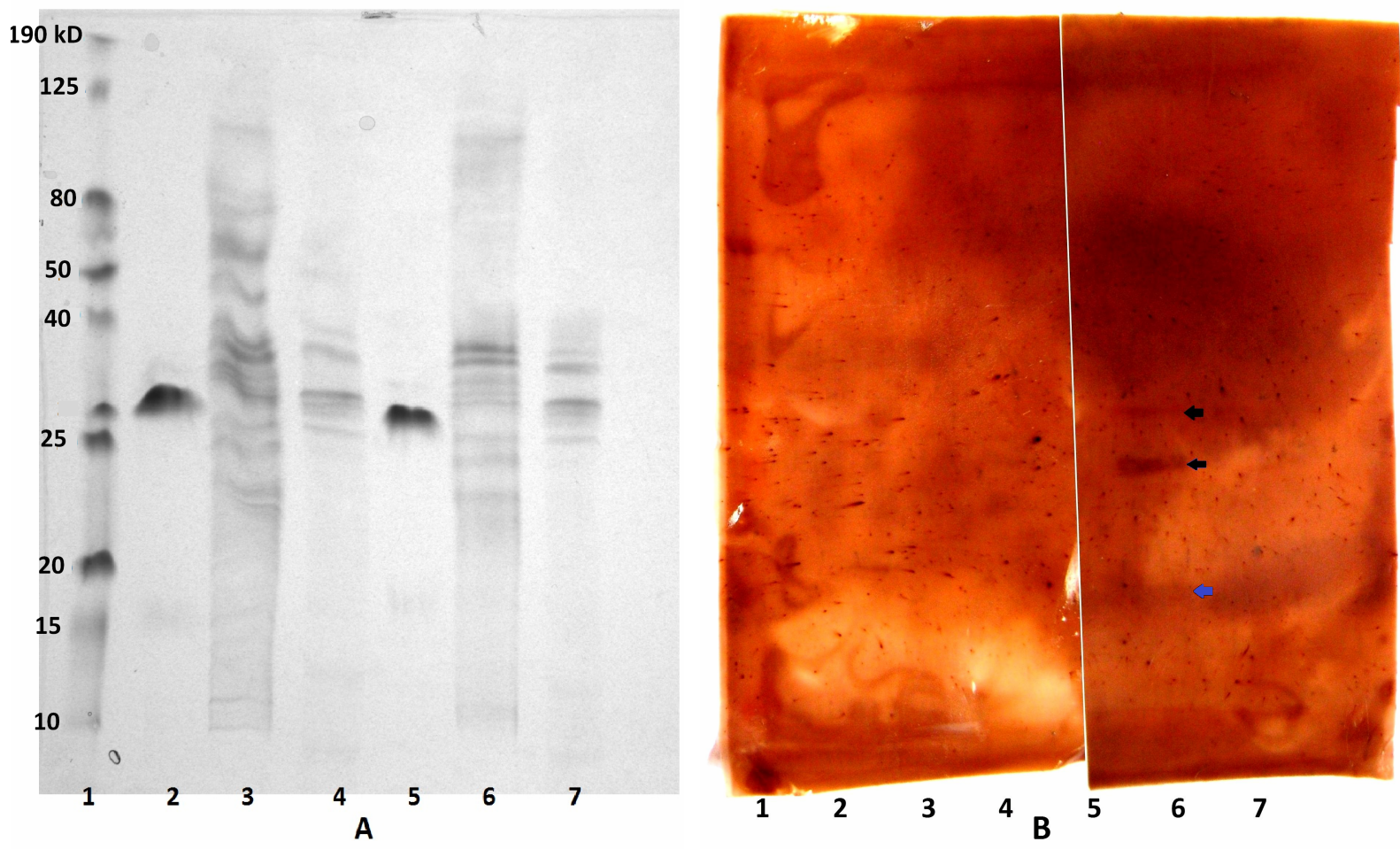

Figure 40. Western blot analysis of negative control, positive control and melanoma tissue. A $1 \%$ Milk Phospholipid $(40 \mu \mathrm{L})$ solution (lanes $1 \& 5)$, a C32 nuclear extract $(37 \mu \mathrm{g})$ (lanes $3 \& 6$ ) and a melanoma whole cell lysate (38.1 $\mu \mathrm{g}$ ) (lanes $4 \& 7$ ) extracted with $2 \%$ SDS protocol and precipitated with TCA-acetone was loaded onto a 4-12\% precast gradient gel $(A)$. The entire gel was used to transfer proteins to the blot for 30 minutes $(B)$. The membrane was then incubated with $5 \mu \mathrm{g} / \mathrm{mL}$ Genetex p21 primary antib0ody, followed by incubation with $8 \mu \mathrm{g} / \mathrm{mL}$ Jackson Immunoresearch HRP-conjugated secondary antibody in either Blotto A (blot B, lanes 1-4) or $0.5 \%$ casein buffer. Notice distortion in Figure A, lane 3 and obscurity of the protein ladder in the blot due to high background staining (Figure B, lane 1). Blue arrow points to potential p21 protein band and black arrows indicate extraneous bands.

The protein p21 is known to accumulate through an increase in p21 mRNA expression, whereas p53 protein accumulation during stress occurs through the reduction of their degradation rate (Qiu et al. 2008). p21 
protein was specifically chosen because its induction is often specific to UV-induced DNA damage. The Western blot results reveal a suppression of p21 expression by milk phospholipids upon UVB exposure, which may translate to a reduction in DNA damage incurred by the tissue. Two possible mechanisms by which sphingolipids may participitate in damage control after UV-induced stress may involve their participation in rebalancing the lipid content on the skin's surface, thus improving physical barrier function, or through a regenerative role involving cell signaling and as sources of second messengers during repair or damage response from induced stress. Indeed, it had been reported that phospholipids promote skin repair and regeneration in damaged skin (e.g. atopic dermatitis) by promoting the synthesis of ceramides, which form protective layers on the skin surface as barrier against irritants while retaining skin moisture. Another mechanism of sphingolipid protection may be through an increase in their cell signaling activity, leading to efficient transduction of extracellular signals to the cell's organelles, which may in turn, effectively induce cell cycle arrest through p21 upregulation that can lead to enhanced DNA repair.

Alternatively, lack of p21 upregulation may also imply failure to initiate cell cycle arrest by milk phospholipids despite having acquired considerable DNA damage, perhaps through indirect effects such as altering the behavior of proteins in the upstream processes. In fact, upregulation of p53 and/or p21 expression is the mechanism of action by a few photoprotective agents such as silbinin (Singh and Agarwal, 2005), green tea and caffeine so as to selectively induce apoptosis in UVB-damaged cells ( $\mathrm{Lu}, 2000 ; \mathrm{Lu}, 2002)$. In another acute exposure procedure, hairless mouse skin showed an increase in p21 protein levels, and since p21 directly binds PCNA to inhibit the latter's function, UVB-induced cell proliferation was inhibited in the epidermal cells (Dhanalakshmi, Mallikarjuna et al. 2004). In general however, upregulation of tumor-suppressor proteins is still a relatively unexplored chemopreventive approach (Smith et al. 2000).

On one hand, an expected decrease in p21 expression may also be supported by findings that p21 expression is induced only after exposure to low UV doses of up to $40 \mathrm{~mJ} / \mathrm{cm}^{2}$ in cultured cells (Murphy et al. 2002; Cotton et al. 1997) and not with higher doses, presumably in order to activate repair. It was further revealed that instead of p21 protein, Bax protein was induced at higher UV doses to initiate the apoptotic pathway through a p53dependent mechanism. These findings suggest that when cells are unable to repair the DNA, apoptosis is triggered as a last resort to avoid the more deleterious effects of mutagenesis and carcinogenesis. Thus, a decrease in p21 expression may, in some cases such as in high UV dose studies, signify increased apoptotic activity in damaged cells rather than the cells acquiring a decreased photodamage from exposure. It is important to keep in mind however, 
that the observed effects of high and low UV doses may not be accurate for intact skin tissues, which may require greater doses than monolayer cultures to achieve the same damaging effects. In addition, this assertion seems to be unlikely given our findings since it does not explain the upregulation of p21 protein observed in our UV samples not incubated with milk phospholipids.

It is also not uncommon that bioactive phytochemicals exert more than one function depending on the extent of damage incurred by the cell. For instance, silibinin (a flavonoid component in milk thistle) is believed to have a dual efficacy in protecting against or promoting apoptosis in keratinocyte cells, thereby acting almost as a damage sensor to decide which cell fate to engage (Dhanalakshmi, Mallikarjuna et al. 2004). To illustrate, silibinin inhibited UVB-induced MAPK activation in nontumorigenic skin cells to prevent proliferation, transformation and apoptosis (Mallikarjuna, Dhanalakshmi et al. 2004). Conversely, it was also capable of promoting apoptosis in tumorigenic skin cells by activating MAPK and cell death related cascades and cause an increase in p53 levels. It is important to note however, that the UVB-induced damage in the former is acute while the latter involved chronic exposure.

Perhaps, another definitive approach to verify whether milk phosphilipids participate in a barrier function or in DNA damage repair is to evaluate the progress of DNA damage over an extended period of time. One immediate marker to explore is thymine dimer formation by immunostaining at different time points after irradiation or by performing DNA damage assays, which note changes in their gel migration behavior as a result of breaks in the strand, denaturation or other structural changes caused by the damage. Decreasing trends of thymine dimer formation can be observed as early as 1 hour after UVB irradiation (Dhanalakshmi et al. 2004) and subsequent decrease in the rate of thymine dimer formation may give an affirmative indication on the possible engagement of a DNA repair system due to the chemopreventive influence of the agent of interest. Also, the milk phospholipid incubation step may be performed after UV irradiation in order to eliminate possible barrier effects and hence, any upregulation in p21 expression observed would denote milk phospholipid's involvement in a DNA damage response, such as repair and anti-proliferation, as opposed to a preventive function. Testing for the expression of p53 alongside p21 may also help to further elucidate the principle behind the observed p21 decrease. In the event that p53 protein is upregulated despite low p21 expression, this may indicate that a divergent damage response pathway downstream of p53, not involving p21 expression, may have been initiated - as in the case of Bax induction of 
apoptosis. On the other hand, simultaneous reduction in both p53 and p21 expression may reinforce the theory that the observed reduction is an effect of reduced photodamage in the tissue.

Eventually, the route of delivery of potential protective agents must also be taken into consideration in the development of chemotherapeutic agents. Since the set-up for milk phospholipid treatment in this study was through the diffusion of milk phospholipids from the media into the membrane, thereby mimicking the oral route of delivery through the blood vessels, issues with inadequate sunscreen application is replaced with challenges in transporting enough concentration of the protective agent to target epidermal regions. However, it is imperative to first conduct studies and come up with approaches that would ensure their ease of passage through the stomach and intestines without degradation and that would ensure their survival in intact or activated form while in circulation. Likewise, several obstacles have to be addressed with topical delivery of chemical agents through the skin. Despite offering easily accessible sites and large surface area that may seem ideal for both local and systemic therapeutic applications, the skin is ultimately designed as a constantly self-repairing barrier that ensures separation between internal and external environments. In more complex analysis of skin structure, the skin stratification can be regarded as a series of physical barriers. Other impediments include the capillaries and lymph ducts, which are efficient at removing molecules within the dermo-epidermal layer, thus reducing the amount of permeants that are capable of reaching the dermis. In a few instances, transdermal drug delivery is restricted by limited metabolic activity that occurs within skin membranes and likewise, immune responses may impede the activity of certain compounds that have otherwise been proven effective in in vitro studies. For instance, topical application of sphingosine was unable to inhibit tumorigenesis in skin (Enkvetchakul, Barnett et al. 1992) despite having exhibited anti-cancer properties in C3H-10T1/2 cells (Borek et al. 1991) and mouse skin (Enkvetchakul et al. 1989).

Extensive studies on the optimal concentration of milk phospholipids that will confer the therapeutic effects also need to be performed since it has been discovered that slight variations in concentration of dietary sphingolipids exert different results. Research performed by Dillehay and colleagues (1994) demonstrated that mice fed with $0.1 \%$ w/w (0.1 g sphingomyelin/100 g diet) purified buttermilk sphingomyelin resulted in a $70 \%$ reduction in colonic crypt foci and $30 \%$ reduction in aberrant crypts per focus, which are premalignant lesions of colon cancer. However, sphingomyelin supplementation at low concentrations of $0.025 \% \mathrm{w} / \mathrm{w}$ sphingomyelin diet actually induced an increase in proliferation of crypt cells. They inferred that this is attributed to the bimodal (Stevens et al. 1990a) and mitogenic effects (Zhang, Buckley et al. 1990) of sphingosine, wherein high concentrations inhibited growth while 
low concentrations promoted proliferation. The authors revealed that they employed this concentration range in their study to mimic sphingomyelin levels normally found in whole milk (Zeisel, Char et al. 1986). Likewise, feeding with $\sim 0.025-0.05 \%$ of the diet was enough $(\boldsymbol{P}=0.075$ to 0.043$)$ to shift the type of tumor from adenocarcinoma to the more benign adenomas. This concentration more closely approximates the normal consumption $(0.01-0.02 \%$ of the diet) in the United States (Vesper, Schmelz et al. 1999). Such findings suggest that slightly increasing the amount of sphingolipids in the diet may further inhibit colon cancer.

Sphinganine and sphingosine are growth stimulatory at low concentrations but cytotoxic at high concentrations (Merrill et al. 1997). Interestingly, use of ceramides, cholesterol or free fatty acid alone, or a mixture of two of these, resulted in delayed barrier recovery (Choi and Maibach, 2005). Only with the mixture of all three lipids, at a specific relative ratio, was barrier recovery accelerated. These results indicate that a balance in the ratio of these three lipids, and not their mere presence, is crucial for skin barrier homeostasis. Barrier recovery was significantly accelerated by topical application of the complete mixture (i.e. ceramides 3 , $3 \mathrm{~B}$, and 6 , together with cholesterol, phytosph ingosine, and linoleic acid) compared with the application of ceramide 3 and $3 \mathrm{~B}$ emulsion. These investigations demonstrated that ceramides combined with other skin lipids improved the repair of skin barrier after damage. 


\section{Chapter V. Conclusion}

Milk phospholipid pre-incubation of skin tissue equivalents induces a reduction in p21 upregulation and development of apoptotic markers such as DNA fragmentation and nuclear condensation, but causes an increase in epidermal proliferation upon UV exposure. Based on current knowledge on the inherent characteristics of phospholipids, it is highly probable that this class of molecules is involved in conferring protection against UVinduced photodamage in skin. The detailed mechanism behind such action is yet to be understood but on the basis of currently known functions of sphingolipid metabolites, it is likely that the effect is due to their role in improving the membrane barrier properties of skin as manifested by an observed increase in epidermal thickness or to their

participation in cell signaling processes during damage response or repair. Nonetheless, further experimentation is necessary before the extent of these effects can be ascertained and their generalized impact on damage prevention and disease therapy can be realized. 


\section{Chapter VI. Future Work and Recommendations}

Extensive research is still necessary before the molecular effects and epidermal fate of milk phospholipids can be completely understood. In terms of improvements in the Western blot technique, duplicate samples are ideal to ensure the reproducibility of results and the validity of data to statistical analysis. Further optimization of steps is beneficial, including the employment of a nuclear extraction protocol to further improve the nuclear to total protein ratio as well as the protein quality and yield. Use of monoclonal antibodies over polyclonal antibodies will help eliminate extraneous bands in the blots. Several 3-in-1 extraction kits have recently been available in the market. For immunofluorescence staining, use of effective antigen retrieval and nuclear permeabilization protocols will be helpful in increasing p21 signal in cells and may eventually permit quantification of relative p21 expression between treatment groups and positive controls through the measurement of signal intensity emitted by the cells. Also, normalization of the Hoechst intensity image is recommended before merging the channels during image analysis in order to eliminate any effects of variable intensity in the Hoechst channel which may interfere with the accurate detection of p21 signal intensity.

A fairly novel technology for biolabeling is the development of quantum dots. Quantum dots of II-VI and III-V semiconductor in the 2-12 $\mathrm{nm}$ range have spawned research interest within the fields of physics, engineering, chemistry and biology. During synthesis, their size and composition can be regulated in order to manipulate their optical properties. Upon absorption of light, quantum dots re-emit the light at a longer wavelength and produce fluorescence. They are ideal for fluorescent tagging of milk phospholipid localization within the cells due to several of their properties: Quantum dots have a broad excitation spectrum yet narrow emission spectrum, are amenable to the precise control of their emmission peak, have long fluorescence lifetime compared to organic fluorophores and can be tailored to any color of a specific wavelength with minimal photobleaching (Liu et al. 2005). Quantum dots may prove useful in tagging phospholipids to visualize their localization within the cell in real-time or in fixed specimens. In conjunction, confocal laser scanning microscopy techniques may also be developed and employed for

high resolution imaging of thick or whole mount tissues in order to visualize relative localization of markers of interest. The instrument is powerful at resolving up to about $140 \mathrm{~nm}$ laterally separated points and has approximately $1 \mu \mathrm{m}$ axial resolution. One of its major advantages is the capacity to image in vitro or in vivo optical 
sections at multiple depths reasonably fast without the need for invasive processing such as fixation and mechanical sectioning.

Further work on melanoma cells or tissues may also help determine if milk phospholipids can also reverse the progression or cause redifferentiation of cancer cells. Alternatively, cultured cells can be used for a more controlled study that would allow identification of the specific carcinogenesis phases that the agent acts upon. For example, in a study conducted by Borek and Merrill (1993) they were able to demonstrate that sphingosine and sphinganine blocks the "promotion" of transformation of C3H 10T1/2 cells by phorbol ester (Borek and Merrill, 1993).

Lastly, evaluating the expression of other biomolecular markers such as PCNA for cell proliferation, stress activated protein kinase/c-Jun kinase, p38 mitogen-activated protein kinase, BrdU-positive cells for specific proliferation in the S phase (DNA synthesis), p27 as proliferation marker similar to p21 (Polyak, 1994) and ornithine decarboxylase for tumor promotion induced by phorbol esters (Merrill et al. 1997) may be performed to further elucidate mechanisms that govern the photoprotective effects. 


\section{Bibliography}

Abdel-Naser MB, Abdallah M, de Almeida Jr. HL and Wollina U. (2005). Human skin cell culture and its impact on dermatology. Egyptian Dermatology Online Journal, 1(2): 1-25.

Aboussekhra A., Biggerstaff M, Shivji MK, Vilpo JA, Moncollin V, Podust VN, Protic M, Hubscher U, Egly JM, Wood RD. (1995). Mammalian DNA nucleotide excision repair reconstituted with purified protein components. Cell, 80:859-868.

Ahmed NU, Ueda M, Nikaido O, Osawa T, Ichihashi M. (1999). High levels of 8-hydroxy-2'deoxyguanosine appear in normal human epidermis after a single dose of ultraviolet radiation. British Journal of Dermatology, 40:226-31.

Ames BN, Gold LS, Willett WC. (1995). The causes and prevention of cancer. Proceedings of the National Academy of Sciences of the United States of America, 92, 5258-5265.

American Academy of Dermatology (AAD) (2011, February 6). Incidence of skin cancer rising at alarming rate. ScienceDaily. Retrieved June 15, 2011, from http://www.sciencedaily.com/releases/2011/02/110205140318.htm

Ananthaswamy HN, Loughlin SM, Cox P, Evans RL, Ullrich SE \& Kripke ML. (1997). Sunlight and skin cancer: inhibition of p53 mutations in UV-irradiated mouse skin by sunscreens. Nature Medicine, 3: 510-14.

Anglin JH, Bever AT, Everett MA and Lamb JH. (1961). Ultraviolet-light-induced alterations in urocanic acid in vivo. Biochimica et Biophysica Acta, 53, 408-409.

Armstrong BK, Kricker A. (1993). How much melanoma is caused by sun exposure? Melanoma Research, 3(6):395-401

Assefa Z, Garmyn M, Bouillon R, Merlevede W, Vandenheede JR, Agostinis P. (1997). "Differential Stimulation of ERK and JNK Activities by Ultraviolet B Irradiation and Epidermal Growth Factor in Human Keratinocytes." Journal of Investigative Dermatology, 108(6): 886-891.

Assefa Z, Vantieghem A, Garmyn M, Declercq W, Vandenbeele P, Vandenheede JR, Bouillon R, Merlevede W, and Agostinis P. (2000). "p38 Mitogen-activated Protein Kinase Regulates a Novel, Caspase-independent Pathway for the Mitochondrial Cytochromec Release in Ultraviolet B Radiation-induced Apoptosis." Journal of Biological Chemistry, 275(28): 21416-21421

Augenstein LG. and Riley P. (1964). The inactivation of enzymes by ultraviolet light. Photochemistry and Photobiology, 3: 353.

Autier P, Boniol M, Severi G and Dore JF. (2001). European Organization for Research and Treatment of Cancer Melanoma Co. Quantity of sunscreen used by European students. British Journal of Dermatology, 144:28891.

Azzi A, Breyer I, Feher M, Pastori M, Ricciarelli R, Spycher S, Staffieri M, Stocker A, Zimmer S and Zingg M. (2000). Specific cellular responses to alpha-tocopherol. Journal of Nutrition, 130:1649-52.

Bacci S, Nakamura T and Streilein JW. (1996). "Failed Antigen Presentation After UVB Radiation Correlates With Modifications of Langerhans Cell Cytoskeleton." Journal of Investigative Dermatology, 107(6): 838-843.

Backvall H, Stromberg S, Gustafsson A, Asplund A, Sivertsson A, Lundeberg J and Ponten F. (2004). Mutation spectra of epidermal p53 clones adjacent to basal cell carcinoma and squamous cell carcinoma. Experimental Dermatology, 2004;13:643-50.

Bale AE and Yu K-p. (2001). The hedgehog pathway and basal cell carcinomas. Human Molecular Genetics, 10(7): 757-762

Barry MA, Eastman A. (1993). Identification of deoxyribonuclease II as an endonuclease involved in apoptosis. Archives of Biochemistry Biophysics, 300:440-450 
Basal and Squamous Cell Carcinoma [Online Photograph]. (2008). Retrieved May 21, 2011 from revolutionhealth.com. http://www.revolutionhealth.com/articles/basal-and-squamous-cell-carcinoma/zm24 40.

Bell RM, Hannun YA and Merrill AH, eds. (1993) Advances in Lipid Research: Sphingolipids and Their Metabolites," vol. 25-26. Academic Press, Orlando, FL.

Birt, D. F., A. H. Merrill, et al. (1998). "Inhibition of skin carcinomas but not papillomas by sphingosine, Nâ€ $\square$ methylsphingosine, and Nâ€ $\square$ acetylsphingosine." Nutrition and Cancer, 31(2): 119-126.

Blank, M. L., Cress, E. A., Smith, Z. L., and Snyder, F. (1992). Meats and fish consumed in the American diet contain substantial amounts of etherlinked phospholipids. Journal of Nutriton. 122, 1656-1661.

Bloom, W. (1965). Electron microscopy of UV-irradiated parts of chromosomes (Amblystoma tigrium). Proceedings of the National Academy of Sciences of the United States of America. 53, 1294-1302.

Blum, H. F. (1959). Carcinogenesis by Ultraviolet Light, Princeton University Press, Princeton, N.J.

Blum, H. F. (1955). Sunburn. In: Radiation Biology II (Ed. by A. Hollaender). McGraw-Hill Book Co., Inc., New York, pp. 487-528.

Bohr VA, Smith CA, Okumoto DS, Hanawalt PC. (1985). DNA repair in an active gene: removal of pyrimidine dimers from the DHFR gene of CHO cells is much more efficient than in the genome overall. Cell, 40:359368.

Boldt S, Weidle UH and Kolch W. (2002). The role of MAPK pathways in the action of chemotherapeutic drugs. Carcinogenesis, 23(11): 1831-1838.

Borek C, and Merrill AH, (1993). Sphingolipids inhibit multistage carcinogenesis and protein kinase C. In Antimutagenesis and Anticarcinogenesis Mechanisms III (G. Bronzetti, M. Hayatsu, S. DeFlora, M. D. Waters, and D. M. Shankel, Eds.), pp. 367-371. Plenum, New York.

Borek C, Ong A, Stevens VL, Wang E and Merrill AH. (1991). Long-chain (sphingoid) bases inhibit multistage carcinogenesis in mouse $\mathrm{C} 3 \mathrm{H} / 10 \mathrm{~T} 1 / 2$ cells treated with radiation and phorbol 12-myristate 13-acetate. Proceedings of the National Academy of Sciences of the United States of America, 88:1953-1957.

Bouwstra JA, Honeywell-Nguyen PL, Gooris GS and Ponec M. (2003). Structure of the skin barrier and its modulation by vesicular formulations. Progress in Lipid Research, 42: 1-36.

Bouwstra JA, Honeywell-Nguyen PL. (2002). Skin structure and mode of vesicles. Advanced Drug Delivery Reviews, 54: s41-55

Bowden GT. (2004). Prevention of non-melanoma skin cancer by targeting ultraviolet-B-light signalling. Nature Reviews Cancer, 4(1): 23-35.

Brachmann RK, Vidal M and Boeke JD. (1996). Dominant-negative p53 mutations selected in yeast hit cancer hot spots. Proceedings of the National Academy of Sciences of the United States of America, 93:4091-4095.

Brash DE, Rudolph JA, Simon JA, Lin A, McKenna GJ, Baden HP, Halperin AJ and Ponten J. (1991). A role for sunlight in skin cancer: UV-induced p53 mutations in squamous cell carcinoma. Proceedings of the National Academy of Sciences of the United States of America, 88(22): 10124-10128

Brash DE, Ziegler A, Jonason AS, Simon JA, Kunala S and Leffell DJ. (1996). Sunlight and sunburn in human skin cancer: p53, apoptosis, and tumor promotion. Journal of Investigative Dermatology Symposium Proceedings, 1(2): 136-142

Burek C, Roth J, Koch HG, Harzer K, Los M and Schulze-Osthoff K. (2001). The role of ceramide in receptor and stress induced apoptosis studied in acidic ceramidase-deficient Farber disease cells. Oncogene, 20: 6493502

Campbell C, Quinn AG, Ro Y-S, Angus B \& Rees JL (1993). p53 mutations are common and early events that precedes tumor invasion in squamous cell neoplasia of the skin. Journal of Investigative Dermatology, $100,746-8$ 
Chen WX, Barthelman M, Martinez J, Alberts D, Gensler HL. (1997). Inhibition of cyclobutane pyrimidine dimer formation in epidermal p53 gene of UV-irradiated mice by alpha-tocopherol. Nutrition and Cancer, 29:205-11.

Choi MJ, Maibach HI (2005) Role of ceramides in barrier function of healthy and diseased skin. American Journal of Clinical Dermatology, 6:215-23

Cifone MG, De Maria R, Roncaioli P, Rippo MR, Azuma M, Lanier LL, Santoni A and Testi R. (1994). Apoptotic signaling through CD95 (Fas/Apo-1) activates an acidic sphingomyelinase. The Journal of Experimental Medicine, 180(4):1547-1552

Clarke AR, Purdie CA, Harrison, DJ, Morris RG, Bird CC, Hooper ML, and Wyllie AH. (1993). Thymocyte apoptosis induced by p53-dependent and independent pathways. Nature (London.), 362:849 - 852.

Cleaver JE \& Kraemer KH. (1989) Xeroderma pigmentosum. In The Metabolic Basis of Inherited Disease, Vol. II (Scriver CR, Beaudet AL, Sly WS, Valle D, eds). New York: McGraw-Hill, 2949-71.

Coderch, L., O. Lopez, et al. (2003). "Ceramides and skin function." American Journal of Clinical Dermatology, 4(2): 107-129.

Cook, J. S. (1965). The quantitative interrelationships between ion fluxes, cell swelling and radiation dose in UV hemolysis. The Journal of General. Physiology, 84, 719-734.

Cotton J, Spandau DF. (1997). Ultraviolet B-radiation dose influences the induction of apoptosis and p53 in human keratinocytes, Radiation Research, 147: 148- 155.

Cummings, B. (2001). Epidermis [Illustration]. Retrieved April 11, 2011 from http://www.highlands.edu/academics/ divisions/scipe/ biology/faculty/harnden/2121/images/integ.jpg

Danno, K. and T. Horio (1987). "Sunburn cell: Factors involved in its formation." Photochemistry and Photobiology, 45(5): 683-690

Darr, D. and I. Fridovich (1994). "Free Radicals in Cutaneous Biology." Journal of Investigative Dermatology, 102(5): 671-675.

Darr D, Combs S, Dunston S, Manning T, Pinnell S. (1992). Topical vitamin C protects porcine skin from ultraviolet radiation-induced damage. British Journal of Dermatology, 127:247-53.

Davidson, J.M., LuValle, P.A., Zoia, O., Quaglino, D. and Giro, M.G. (1997). Ascorbate differentially regulates elastin and collagen biosynthesis in vascular smooth muscle cells and skin fibroblasts by pretranslational mechanisms. The Journal of Biological Chemistry, 272: 345-352.

Daya-Grosjean, L., Dumaz, N., Sarasin, A. (1995). The specificity of p53 mutation spectra in sunlight-induced human cancers. Journal of Photochemistry and Photobiology, 28: 115-124.

DeBuys HV, Levy SB, Murray JC, Madey DL, Pinnell SR. (2000). Modern approaches to photoprotection. Dermatologic Clinics, 18:577-90.

De Fabo EC and Noonan FP. (1983). Mechanism of immune suppression by ultraviolet irradiation in vivo. I. Evidence for the existence of a unique photoreceptor in skin and its role in photoimmunology. The Journal of Experimental Medicine, 158, 84-98.

de Gruijl FR, van der Leun JC. (1994). Estimate of the wavelength dependency of ultraviolet carcinogenesis in humans and its relevance to the risk assessment of stratospheric ozone depletion. Health Physics, 67:31925 .

Delgado JA, Anasagasti L, Quesada I, Cruz JC, and Joan AY. "Ex-vivo Autofluorescence Measurements of Human Tissues", in VII Mexican Symposium on Medical Physics-2003, accepted for publication

Delgado JA, Rubiera L, and Anasagasti L. "Overview of Instrumentation for Diagnosis of Cancer using Laser Induced Fluorescence Spectroscopy", in Sixth Mexican Symposium on Medical Physics-2002, edited by L.M. Monta no et al. (AIP Conferences Proceedings 630, Melville, New York, 2002) p. 185. 
Denning MF, Wang Y, Nickoloff BJ and Wrone-Smith T. (1998). Protein Kinase CÎ́ Is Activated by Caspasedependent Proteolysis during Ultraviolet Radiation-induced Apoptosis of Human Keratinocytes. Journal of Biological Chemistry, 273(45): 29995-30002

"DeRiPRO, DNA, RNA and Proteins Extraction Technology," PCT/MY2008/000059, http://terraju.com/TLS.htm.Lane, D. P. p53 guardian of the genome. Nature (Lond.), 358: 15-16, 1992

Desal ID, Sawant PL and Tappel AL. (1964). Peroxidative and radiation damage to isolated lysosomes. Biochimica et. Biophysica Acta, 86, 277-285.

Dhanalakshmi S, Mallikarjuna GU, Singh RP and Agarwal R. (2004). Silibinin prevents ultraviolet radiation-caused skin damages in SKH-1 hairless mice via a decrease in thymine dimer positive cells and an up-regulation of p53-p21/Cip1 in epidermis. Carcinogenesis, 25(8): 1459-1465

Dhanalakshmi S, Mallikarjuna GU, Singh RP and Agarwal R. (2004). Dual efficacy of silibinin in protecting or enhancing ultraviolet $\mathrm{B}$ radiation-caused apoptosis in $\mathrm{HaCaT}$ human immortalized keratinocytes. Carcinogenesis, 25(1): 99-106

Dillehay DL, Webb SK, Schmelz EM an Merrill AH. (1994). Dietary sphingomyelin inhibits 1,2dimethylhydrazine-induced colon cancer in CF1 mice. Journal of Nutrition, 124(5): 615-620.

Dobson RL. (1963). Anthramine carcinogenesis in the skin of rats. 1. The epidermis. Journal of the National Cancer Institute, 31, 841-859.

Donahue BA, Yin S, Taylor JS, Reines D \& Hanawalt PC. (1994). Transcript cleavage by RNA polymerase II arrested by a cyclobutane pyrimidine dimer in the DNA template. Proceedings of the National Academy of Sciences of the United States of America, 91, 8502-6.

Dudeja PK, Dahiya R and Brasitus TA. (1986) The role of sphingomyelin synthase and sphingomyelinase in 1,2dimethylhydrazine-induced lipid alterations of rat colonic plasma membranes. Biochimica et Biophysica Acta, 863: 309-312.

Duthie MS, Kimber I and Norval M. (1999). The effects of ultraviolet radiation on the human immune system. British Journal of Dermatology, 1999;140:995-1009.

Dutta A, Ruppert JM, Aster JC and Winchester E. (1993). Inhibition of DNA replication factor RPA by p53. Nature, $365,79-82$.

Eisinger M, Marko O. (1982). Selective proliferation of normal human melanocytes in vitro in the presence of phorbol ester and cholera toxin. Proceedings of the National Academy of Sciences of the United States of America, 79:2018-2022.

el-Deiry WS, Tokino T, Velculescu VE, Levy DB, Parsons R, Trent JM, Lin D, Mercer WE, Kinzler KW and Vogelstein B. (1993). WAF1, a potential mediator of p53 tumor suppression. Cell, 75:817-825.

el-Deiry WS, Tokino T, Waldman T, Oliner JD, Velculescu VE, Burrell M, Hill DE, Healy E, Rees JL Hamilton SR, Kinzler KW and Vogelstein B. (1995). Topological control of p21WAF1/CIP1 expression in normal and neoplastic tissues. Cancer Research, 55:2910 \pm 2919 .

Electromagnetic spectrum [Online Image]. (n.d.). Retrieved May 21, 2011, from NASA.gov. http://www.nasa.gov/a udience/forstudents/k-4/dictionary/Electromagnetic_Spectrum.html

Eller MS, Gilchrest BA. (2000). Tanning as part of the eukaryotic SOS response. Pigment Cell Res, 13:94-7.

Endo K, Igarashi Y, Nisar M, Zhou Q and Hakomori S. (1991). Cell Membrane Signaling as Target in Cancer Therapy: Inhibitory Effect of N,N-Dimethyl and N,N,N-Trimethyl Sphingosine Derivatives on in Vitro and in Vivo Growth of Human Tumor Cells in Nude Mice. Cancer Research, 51(6): 1613-1618

Endo M, Igarashi Y, Nisar M, Zhou AQ and Hakomori S. (1991) Cell membrane signalling as target in cancer therapy: Inhibitory effect of N,A/-dimethyl and N,N,N-trimethy $\backslash$ sphingosine derivatives on in vitro and in vivo growth of human tumor cells in nude mice. Cancer Research, 51: 1613-1618. 
Enkvetchakul B, Merrill AH, and Birt DF. (1989). Inhibition of the induction of ornithine decarboxylase activity by 12-O-tetradecanoylphorbol-13-acetate in mouse skin by sphingosine sulfate. Carcinogenesis, 10(2): 379381

Enkvetchakul B, Barnett T, Liotta DC, Geisler V, Menaldino D, Merrill AH and Birt DF. (1992). Influences of sphingosine on two-stage skin tumorigenesis in Sencar mice. Cancer Letters, 62(1): 35-42

European Centre for the Validation of Alternative Methods. (2009). Performance Standards for In-Vitro Skin Irritation Test Methods based on Reconstructed Human Epidermis (RhE). Retrieved July 9, 2011 from http://ecvam.jrc.it/ft_doc/ECVAM_PS_invitro_Skin_Irritation_0908-26.pdf.

Evan GI and Vousden KH. (2001). Proliferation, cell cycle and apoptosis in cancer. Nature, 411(6835): 342-348.

Fisher GJ, Choi HC, Bata-Csorgo Z, Shao Y, Datta S, Wang ZQ, et al. (2001). Ultraviolet irradiation increases matrix metalloproteinase-8 protein in human skin in vivo. Journal of Investigative Dermatology, 117:21926.

Fisher GJ, Datta SC, Talwar HS, Wang ZQ, Varani J, Kang S, et al. (1996). Molecular basis of sun-induced premature skin aging and retinoid antagonism. Nature, 379:335-9.

Fisher GJ, Datta S, Wang ZQ, Li XY, Quan TH, Chung JH, et al. (2000). c-Jun-dependent inhibition of cutaneous procollagen transcription following ultraviolet irradiation is reversed by alltrans retinoic acid. The Journal of Clinical Investigation, 106:663-70.

Fisher GJ, Wang ZQ, Datta SC, Varani J, Kang S and Voorhees JJ. (1997). Pathophysiology of premature skin aging induced by ultraviolet light. The New England Journal of Medicine, 337:1419-29.

Flores-Rozas H, Kelman Z, Dean FB, Pan ZQ, Harper JW, Elledge SJ, O’Donnell MO and Hurwitz J. (1994). Cdkinteracting protein I directly binds with proliferating cell nuclear antigen and inhibits DNA replication catalyzed by the DNA polymerase delta holoenzyme. Proceedings of the National Academy of Sciences of the United States of America, 91: 8655-8659

Fotedar R, Bendjennat M, et al. (2004). Role of p21WAF1 in the Cellular Response to UV. Cell Cycle, 3(2): 132135 .

Friedberg EC. (2001). How nucleotide excision repair protects against cancer. Nature Reviews Cancer 1(1): 22-33.

Geilen CC, Wieder $\mathrm{T}$ and Orfanos CE. (1997.) Ceramide signalling: regulatory role in cell proliferation, differentiation and apoptosis in human epidermis. Archives of Dermatological Research, 289 (10): 559-66

Gensler HL, Magdaleno M. (1991). Topical vitamin E inhibition of immunosuppression and tumorigenesis induced by UV irradiation. Nutrition and Cancer, 15:97-110.

Ghosh TK, Bian J, et al. (1990). Intracellular Calcium Release Mediated by Sphingosine Derivatives Generated in Cells. Science, 248(4963): 1653-1656.

Giese AC. (1938). Differential susceptibility of a number of protozoans to ultraviolet radiation. Journal of Cellular and Comparative Physiology, 12:129-38.

Giese AC and Reed EA. (1940). Ultraviolet radiations and cell division. Variation in resistance to radiations with stock, species, and nutritional differences in Paramecium. Journal of Cellular and Comparative Physiol, 15:395-408.

Gilchrest BA, Vrabel MA, Flynn E, Szabo G. (1984). Selective cultivation of human melanocytes from newborn and adult epidermis. Journal of Investigative Dermatology, 83:370-376.

Gloster HM \& Brodland DG. (1996). The epidemiology of skin cancer. Dermatologic Surgery, 22, 217-26.

Gobert C, Bracco L, Rossi F, Olivier M, Tazi J \& Lavell F. (1996). Modulation of DNA topoisomerase activity by p53. Biochemistry, 35, 5578-86.

Gomez-Munoz A, Martin A, O'Brien L, and Brindley DN. (1994). Cell-permeable ceramides inhibit the stimulation of DNA synthesis and phospholipase D activity by phosphatidate and lysophosphatidate in rat fibroblasts. Biological Chemistry, 69, 8937-8943 
Gonzalez, S. et al. (1996). Development of cutaneous tolerance to ultraviolet B during ultraviolet B phototherapy for psoriasis. Photodermatology, Photoimmunology, Photomedicine, 12(2): 73-78.

Gottlieb RA, Giesing HA, Zhu JY, Engler RL, Babior BM. (1995). Cell acidification in apoptosis: granulocyte colony-stimulating factor delays programmed cell death in neutrophils by up-regulating the vacuolar H1ATPase. Proceedings of the National Academy of Sciences of the United States of America, 92: 5965-5968

Goukassian, D. A., M. S. Eller, et al. (1999). Thymidine Dinucleotide Mimics the Effect of Solar Simulated Irradiation on p53 and p53-Regulated Proteins. Journal of Investigative Dermatology, 112(1): 25-31.

Goukassian D, Gad F, Yaar M, Eller MS, Nehal US, Gilchrest BA. (2000). Mechanisms and implications of the ageassociated decrease in DNA repair capacity. FASEB J,14,1325-34.

Grady, H. G., Blum, H. F. and Kirby-Smith, J. S. (1943). Types of tumor induced by ultraviolet radiation and factors influencing their relative incidence. Journal of the National Cancer Institute, 3, 371-378.

Green D and Kroemer G. (1998). The central executioners of apoptosis: caspases or mitochondria? Trends in Cell Biology, 8(7): 267-271

Greenblatt MS, Bennett WP, et al. (1994). Mutations in the p53 Tumor Suppressor Gene: Clues to Cancer Etiology and Molecular Pathogenesis. Cancer Research, 54(18): 4855-4878.

Griffiths AJF, Gelbart WM, Miller JH and Lewontin RC. (1999). Modern Genetic Analysis. New York: W. H. Freeman.

Gruber JV, Padyachi V, Lods L, Benhaim M, Holtz R, Natalizio A. (2004). Influence of Soybean Symbiosome Extract on UV-Induced Free-Radical Chemistry. Bioinnovation Laboratories, Inc. October 24-2.

Halaban R, Pomerantz S, Marshall S, Lerner AB. (1984). Tyrosinase activity and abundance in Cloudman melanoma cells. Archives of Biochemistry and Biophysics, 230:383-387.

Hale DM, Cromartie WJ and Dobson RL. (1960). Luxol fast blue as a selective stain for dermal collagen. Journal of. Investigative. Dermatology. 35, 293-295.

Hanawalt PC. (1991) Heterogeneity of DNA repair at the gene level. Mutatation. Research, 247, 203-211

Hannun YA, Loomis CR, Merrill AH and Bell RM. (1986). Sphingosine inhibition of protein kinase C activity and of phorbol bibutyrate binding in vitro and in human platelets. The Journal of Biological Chemical, 261: 12604-12609.

Harper JW, Adami GR, Wei N, Keyomarsi K and Elledge SJ (1993) The p21-Cdkinteracting protein Cip1 is a potent inhibitor of G1 cyclin-dependent kinases. Cell, 75: 805-816

Harper JW, Adami GR, et al. (1993). The p21 Cdk-interacting protein Cip1 is a potent inhibitor of G1 cyclindependent kinases. Cell, 75(4): 805-816

Harris, CC. (1996). Structure and Function of the p53 Tumor Suppressor Gene: Clues for Rational Cancer Therapeutic Strategies. Journal of the National Cancer Institute, 88(20): 1442-1455

Hausser, I. (1939). Naturwiss, 27, 563.

Hanahan D,Weinberg RA. (2000). The hallmarks of cancer. Cell, 100:57-70, 2000

Haynes RH. (1964). Role of DNA repair mechanisms in microbial inactivation and recovery phenomena. Photochemistry and. Photobiology. 3, 429-450.

Hengst L, Reed SI. 1996. Translational control of p27 Kip1 accumulation during the cell cycle. Science, 271:186164

Hertervig E, Nilsson A, et al. (1997). Alkaline sphingomyelinase activity is decreased in human colorectal carcinoma. Cancer, 79(3): 448-453.

Herzinger T, Funk JO, Hillmer K, Eick D, Wolf DA \& Kind P. (1995). Ultraviolet B irradiation-induced G2 cell cycle arrest in human keratinocytes by inhibitory phosphorylation of the cdc2 cell cycle kinase. Oncogene, $11,2151-56$. 
Hill LL, Ouhtit A, et al. (1999). Fas Ligand: A Sensor for DNA Damage Critical in Skin Cancer Etiology. Science, 285(5429): 898-900

Huang LC, Clarkin KC, et al. (1996). Sensitivity and selectivity of the DNA damage sensor responsible for activating p53-dependent G1 arrest. Proceedings of the National Academy of Sciences of the United States of America, 93(10): 4827-4832

Huwiler A, Kolter T, Pfeilschifter J and Sandhoff K. (2000). Physiology and pathophysiology of sphingolipid metabolism and signaling. Biochimica et Biophysica Acta, 1485, 63-99.

Ichihashi M, Funasaka Y, Ahmed NU, Chakraborty AK, Ueda M, (1998). Efficacy of antioxidant substances and prevention of UV damage, in symposium on skin: In: J.A. Parrish, H. Tagami, T. Ozawa (Eds.), Interface of a Living System, Elsevier, Amsterdam, pp. 167-175.

Ichihashi M, Funasaka Y, Ohashi A, Chacraborty A, Ahmed NU, Ueda M, et al. (1999).The inhibitory effect of DLalpha-tocopheryl ferulate in lecithin on melanogenesis. Anticancer Research, 19: 3769-74.

Ichihashi M, Ueda M, Budiyanto A, Bito T, Oka M, Fukunaga M, Tsuru K, Horikawa T. (2003). UV-induced skin damage. Toxicology, 189(1-2):21-39.

Ikushima T and Wolff S. (1974). UV-induced chromatid aberrations in cultured Chinese hamster cells after one, two, or three rounds of DNA replication. Mutation Research/Fundamental and Molecular Mechanisms of Mutagenesis, 22(2): 193-201.

Imaizumi K,Tominaga A, Sato M and Sugano M. (1992) Effects of dietary sphingolipids on levels of serum and liver lipids in rats. Nutrition Research, 12(4-5): 543-548.

Imokawa G, Abe A, Jin K, et al. (1991). Decreased level of ceramides in stratum corneum of atopic dermatitis: an etiologic factor in atopic dry skin. Journal of Investigative Dermatology, 96: 523-6

Invitrogen. (2005). Hoechst Stains. Retrieved September 7, 2011 from: http://probes.invitrogen.com/media/pis/mp21 486.pdf

Ito K and Kawanishi S, (1997). Site-specific DNA damage induced by UVA radiation in the presence of endogenous photosensitizer. The Journal of Biological. Chemistry, 378, 1307_/1312.

Jacobs LS, and Kester M. (1993). Sphingolipids as mediators of effects of platelet-derived growth factor in vascular smooth muscle cells. American Journal of Physiology, 65, c740-c747

Jarvis, W. D., F. A. Fornari, Jr., et al. (1996). "Induction of Apoptosis and Potentiation of Ceramide-mediated Cytotoxicity by Sphingoid Bases in Human Myeloid Leukemia Cells." Journal of Biological Chemistry, 271(14): 8275-8284

Jarvinen R, P. Knekt, T. Hakulinen \& A. Aromaa. (2001). Prospective study on milk products, calcium and cancers of the colon and rectum. European Journal of Clinical Nutrition, 55:1000-1007.

Jensen RG, Ferris AM and Lammi-Keefe CJ. (1991). The composition of milk fat. Journal of Dairy Science, 74, 3228-3243.

Jiang H, Lin J, Su ZZ, Herlyn M, Kerbel RS, Weissman BE, Welch DR and Fisher PB. (1995). The melanoma differentiation-associated gene mda-6, which encodes the cyclin-dependent kinase inhibitor p21, is differentially expressed during growth, differentiation and progression in human melanoma cells. Oncogene, 10(9):1855-1864.

Jonason, A. S., S. Kunala, et al. (1996). Frequent clones of p53-mutated keratinocytes in normal human skin. Proceedings of the National Academy of Sciences of the United States of America, 93(24): 14025-14029

Jurkiewicz BA, Bissett DL, Buettner GR. (1995). Effect of topically applied tocopherol on ultraviolet radiationmediated free radical damage in skin. Journal of Investigative Dermatology, 104:484-8.

Kamili A, Wat E, Chung RWS, Tandy S, Weir JM, Meikle PJ and Cohn JS. (2010). Hepatic accumulation of intestinal cholesterol is decreased and fecal cholesterol is increased in mice fed a high-fat diet supplemented with milk phospholipids. Nutrition \& Metabolism, 7:90 
Kanjilal S and Ananthaswamy HN. (1996). Molecular biology of skin carcinomas. In: Basal and squamous cell skin cancers of the head and neck. Eds: Weber R., Miller M., Goepfert H., Williams and Wilkins, Baltimore 2526.

Kanjilal S, Pierceall WE, Cummings KK, Kripke ML \& Ananthaswamy HN. (1993). High frequency of p53 mutations in ultraviolet radiation-induced murine skin tumors: evidence for strand bias and tumor heterogeneity. Cancer Research, 53, 2961-64.

Kaplan HS and Zavarine R. (1962). Correlation of bacterial radiosensitivity and DNA base compositionen. Biochemical and Biophysical Research Communication, 8, 432-436.

Kastan MB, Onyekwere O, et al. (1991). Participation of p53 protein in the cellular response to DNA damage. Cancer Research, 51(23 Pt 1): 6304-6311.

Kastan MB, Zhan Q, el-Deiry WS, et al. (1992). A mammalian cell cycle checkpoint pathway utilizing p53 and GADD45 is defective in ataxia-telangiectasia. Cell, 71:587-597.

Katiyar SK, Korman NJ, Mukhtar H, et al. (1997). Protective effects of silymarin against photocarcinogenesis in a mouse skin model. Journal of the National Cancer Institute, 89, 56-66.

Katz A and Alfano RR, (1996). Photonic pathology - fluorescence and Raman spectrosocopy for tissue diagnosis and characterization. In Analytical Use of Fluorescent Probes in Oncology (Edited by Kohen E and Hirschberg JG) Plenum Press, New York.

Kawaguchi Y, Tanaka H, Okada T, Konishi H, Takahashi M, Ito M, et al. (1997). Effect of reactive oxygen species on the elastin mRNA expression in cultured human dermal fibroblasts. Free Radical Biology and Medicine, 23:162-5.

Kelner A. (1953). Growth, respiration and nucleic acid synthesis in ultraviolet-irradiated and in photoreactivated Escherichia coli. Journal of Bacteriology. 66, 252-262.

Kelly G. (2009). Sphingomyelin conversion cycle [Online Image]. Retrieved August 8 , 2011 from http://www.kellymusings.com/phenoxodiol/

Kichina JV, Rauth S, Das Gupta TK, Gudkov AV. (2003). Melanoma cells can tolerate high levels of transcriptionally active endogenous p53 but are sensitive to retrovirus-transduced p53. Oncogene, 22: 4911 -4917 .

Kielbassa C, Roza L, Epe B. (1997). Wavelength dependence of oxidative DNA damage induced by UV and visible light. Carcinogenesis, 18:811-6.

Kivirikko KI, Myllyla R. (1985). Post-translational processing of procollagens. Annals of the New York Academy of Sciences, 460:187-201.

KNMI / TEMIS. (2006). The CIE action spectrum is a model for the susceptibility of the caucasian skin to sunburn (reddening of the skin; erythema) [Online Image]. Retrieved August 6, 2011 from http://www.temis.nl/uvradiation/info/uvindex.html

Kolesnick R. (2002). The therapeutic potential of modulating the ceramide/sphingomyelin pathway. The Journal of Clinical Investigation, 110: 3-8.

Kraemer KH. (1997). Sunlight and skin cancer: another link revealed. Proceedings of the National Academy of Sciences of the United States of America, 94:11-14.

Kress S, Sutter C, Strickland PT, Mukhtar H, Schweitzer J \& Schwarz M. (1992). Carcinogen-specific mutational pattern in the p53 gene in ultraviolet B radiation-induced squamous cell carcinomas of mouse skin. Cancer Res, 52, 6400-3

Kripke ML. (1981). Immunologic mechanisms in UV radiation carcinogenesis. Adv Cancer Res, 34, 69-106

Kuerbitz SJ, Plunkett BS, Walsh WV, and Kastan MB. (1992). Wild-type p53 is a cell cycle checkpoint determinant following irradiation. Proceedings of the National Academy of Sciences of the United States of America, 89: 7491-7495. 
Kunkel TA. (2004). DNA Replication Fidelity. Journal of Biological Chemistry, 279(17): 16895-16898.

de Laat A, Kroon ED \& de Gruijl FR. (1997). Cell cycle effects and concomitant p53 expression in hairless murine skin after longwave UVA $(365 \mathrm{~nm})$ irradiation: a comparison with UVB irradiation. Photochemistry and Photobiology, 65, 730-35

Le, Anh. (2002). Gradient-gel Electrophoresis in the Clinical Evaluation of Heart Disease Risk. Retrieved July 19, 2011 from http://www.ivdtechnology.com/article/gradient-gel-electrophoresis-clinical-evaluation-heartdisease-risk.

Leverkus M, Yaar M, et al. (1997). "Fas/Fas Ligand Interaction Contributes to UV-Induced Apoptosis in Human Keratinocytes." Experimental Cell Research, 232(2): 255-262

Levine M, Wang YH, Padayatty SJ, Morrow J. (2001). A new recommended dietary allowance of vitamin C for healthy young women. Proceedings of the National Academy of Sciences of the United States of America, 98:9842-6.

Levitt NC, Hickson ID, 2002. Caretaker tumour suppressor genes that defend genome integrity. Trends. Mol. Med. 8, 179-186.

Li J, Eastman A. (1995). Apoptosis in an interleukin-2-dependent cytotoxic T lymphocyte cell line is associated with intracellular acidification. Journal of Biological Chemistry, 270:3203-3211

Liu T, Liu B, Zhang H and Wang Y. (2005). The fluorescence bioassay platforms on quantum dots nanoparticles. Journal of Fluorescence, 15(5):729-733.

Lockshin RA and Zakeri ZF. (1990). Programmed cell death: New thoughts and relevance to aging. Journal of Gerontology, 45, B135-140.

Lopez-Torres M, Thiele JJ, Shindo Y, Han D, Packer L. (1998). Topical application of _tocopherol modulates the antioxidant network and diminishes ultraviolet-induced oxidative damage in murine skin. $\mathrm{Br} J$ Dermatol, 138:207-15.

Lowe SW, Schmitt EM, Smith SW, Osborne BA, and Jacks, T. (1993). p53 is required for radiation-induced apoptosis in mouse thymocytes. Nature (London.), 362: 847-849.

Lu YP, Lou YR, Li XH, Xie JG, Brash D, Huang MT, Conney AH. (2000). Stimulatory effect of oral administration of green tea or caffeine on ultraviolet lightinduced increases in epidermal wild-type p53, p21 (WAF1/CIP1), and apoptotic sunburn cells in SKH-1 mice. Cancer Research, 60, 4785_/4791.

Lu YP, Lou YR, Lin Y, et al. (2001). Inhibitory effects of orally administered green tea, black tea, and caffeine on skin carcinogenesis in mice previously treated with ultraviolet B light (highrisk mice): relationship to decreased tissue fat. Cancer Research, 61, 5002-5009.

Lu Y-P, Lou Y-R, Xie J-G, Peng Q-Y, Liao J, Yang CS, et al. (2002). Topical applications of caffeine or (--)epigallocatechin gallate (EGCG) inhibit carcinogenesis and selectively increase apoptosis in UVB-induced skin tumors in mice. Proceedings of the National Academy of Sciences of the United States of America, 99:19,12455-60.

Luger TA. and Schwarz T. (1990). Evidence for an epidermal cytokine network. Journal of Investigative Dermatology, 95:100S-104S.

MacKenzie A, Vyssotski M and Nekrasov E. (2009). Quantitative analysis of dairy phospholipids by ${ }^{31}$ P NMR. Journal of the American Oil Chemists' Society, 86:757-763

Mallikarjuna G, Dhanalakshmi S, et al. (2004). Silibinin Protects against Photocarcinogenesis via Modulation of Cell Cycle Regulators, Mitogen-Activated Protein Kinases, and Akt Signaling. Cancer Research, 64(17): 6349-6356

Maeda K, Fukuda M. Arbutin. (1996). mechanism of its depigmenting action in human melanocyte culture. Journal of Pharmacology and Experimental Therapeutics, 276:765-9. 
Man MM, Feingold KR, Thornfeldt CR, et al. (1996). Optimization of physiological lipid mixtures for barrier repair. Journal of Investigative Dermatoogyl, 106: 1096-101

Marshall C. (1999). How do small GTPase signal transduction pathways regulate cell cycle entry? Current Opinion in Cell Biology, 11(6): 732-736

Mathias S, L.A. Peña \& R.N. Kolesnick. (1998). Signal transduction of stress via ceramide. Biochemical Journal, $335,465-480$.

Matsumura Y and Ananthaswamy HN. (2002). Short-term and long-term cellular and molecular events following UV irradiation of skin: implications for molecular medicine. Expert Reviews in Molecular Medicine, 4(26): $1-22$

MatTek Corporation. (2007). Epiderm ${ }^{T M}$ cell culture insert. Retrieved June 3, 2011 from http://www.zet.or.at/sub node,2,128,de,EpiDerm\%E2\%84\%A2_Hautirritations_Studie,lifescience.php

McKenzie R, Connor B, Bodeker G. (1999). Increased summertime UV radiation in New Zealand in response to ozone loss. Science, 285, 1709-1711

McLaren AD and Shugar D. (1964). Photochemistry of Proteins and Nucleic Acids. N.Y: Macmillan.

Mehregan AH, Staricco RG and Pinkus H. (1964). Elastic fibers in basal cell epithelioma. Archives of Dermatology, $89,33$.

Melanoma. (2011). Retrieved June 15, 2011, from http://www.cancer.gov/cancertopics/types/melanoma

Merrill AH, Schmelz EM, et al. (1995). Role of dietary sphingolipids and inhibitors of sphingolipid metabolism in cancer and other diseases. Journal of Nutrition, 125(6 Suppl): 1682

Merrill HA, et al. (1997). Sphingolipids : The enigmatic lipid class : Biochemistry, physiology, and pathophysiology. Amsterdam, PAYS-BAS, Elsevier

Merrill AH, Sandhoff K, et al. (2002). Chapter 14 Sphingolipids: metabolism and cell signaling. New Comprehensive Biochemistry, 36: 373-407.

Michieli P, Chedid M, Lin D, Pierce JH, Mercer WE and Givol D (1994) Induction of WAF1/CIP1 by a p53independent pathway. Cancer Research, 54: 3391-3395

Miller DL, Weinstock MA:Non-melanoma. (1994). skin cancer in the United States: incidence. Journal of the American Academy of Dermatology, 30:774-778.

Miller L. (2010). Analyzing gels and Western blots with ImageJ. Retrieved August 9, 2011 from: http://lukemiller.org/index.php/2010/11/analyzing-gels-and-western-blots-with-image-j/

Mitchell DL, Greinert RD, et al. (1999). Effects of Chronic Low-Dose Ultraviolet B Radiation on DNA Damage and Repair in Mouse Skin. Cancer Research, 59(12): 2875-2884

Mitchell DL, Haipek CA, Clarkson JM, (1985). (6-/4) Photoproducts are removed from the DNA of UV-irradiated mammalian cells more efficiently than cyclobutane pyrimidine dimers. Mutation Research, 143, 109-112.

Mitchell DL and Nairn RS. (1989). The biology of the (6-4) photoproduct, Photochemistry and Photobiology, 49, 805-819

Moebius J, Zahedi RP, Lewandrowski U, Berger C, Walter U and Sickmann A. (2005). The human platelet membrane proteome reveals several new potential membrane proteins. Molecular Cell Proteomics, 4(11): 1754-61.

Moglich A, Krieger F, Kiefhaber T. (2005). Molecular basis for the effect of urea and guanidinium chloride on the dynamics of unfolded polypeptide chains. Journal of Molecular Biology. 7; 345(1): 153-62

Molecular view of the cell membrane. [Art]. In Encyclopædia Britannica. Retrieved June 22, 2011 from http://www.britannica.com/EBchecked/media/45550/A-molecular-view-of-the-cell-membraneIntrinsic-proteins-penetrate 
Moller P, Wallin H, Holst E, Knudsen LE. (2002). Sunlight-induced DNA damage in human mononuclear cells. FASEB J, 16:45-53.

Morliere P, Moysan A, Tirache I. (1995). Action spectrum for UV-induced lipid peroxidation in cultured human skin fibroblasts. Free Radical Biology \& Medicine, 19:365-71.

Mowry RW. (1958). Improved procedures for staining of acid polysaccharides by Mueller' choloidal (hydrous) peroxide and its combination with Feulgen and periodic acid-Schiff reactions. Laboratory Investigation. 7, 566.

Munne-Bosch S, Alegre L. (2002). The function of tocopherols and tocotrienols in plants. Critical Reviews in Plant Sciences, 21:31-57.

Murphy M, Mabruk MJ, Lenane P et al. (2002). Comparison of the expression of p53, p21, Bax and the induction of apoptosis between patients with basal cell carcinoma and normal controls in response to ultraviolet irradiation. Journal of Clinical Pathology, 55:829-33.

Nakamura T, Pinnell SR, Darr D, Kurimoto I, Itami S, Yoshikawa K, et al. Vitamin C. (1997). abrogates, the deleterious effects of UVB radiation on cutaneous immunity by a mechanism that does not depend on TNFa. Journal of Investigative Dermatology, 109:20-4.

Nakazawa H, English D, et al. (1994). UV and Skin Cancer: Specific p53 Gene Mutation in Normal Skin as a Biologically Relevant Exposure Measurement. Proceedings of the National Academy of Sciences of the United States of America, 91(1): 360-364

Nativ O, Sabo E, Raviv G, Medalla O, Moskovitz B and Goldwasser B. (1995). The role of nuclear morphometry for predicting disease outcome in patients with localized renal cell carcinoma. Cancer, 76: 1440-1444.

Nay SL, Canning MT, Yarosh DB. (2006). Topically Applied Liposome-Encapsulated T4 Endonuclease V Increases Repair of UV-Induced Cyclobutane Pyrimidine Dimers in Normal Repair Proficient Reconstructed Skin. Society for Investigative Dermatology Annual Meeting. May 3-6, 2006.

Nemes Z, Steinert PM. (1999). Bricks and mortar of the epidermal barrier. Exp Mol Med, 31:5-19.

Nickoloff BJ, Qin JZ, Chaturvedi V, Bacon P, Panella J, Denning MF. (2002). "Life and Death Signaling Pathways Contributing to Skin Cancer." Journal of Investigative Dermatology Symposium Proceedings, 7(1): 27-35.

Nilsson A. (1968). Metabolism of sphingomyelin in the intestinal tract of the rat. Biochimica et Biophysica Acta (BBA) - Lipids and Lipid Metabolism, 164(3): 575-584.

Nishikimi M, Fukuyama R, Minoshima S, Shimizu N, Yagi K. (1994). Cloning and chromosomal mapping of the human nonfunctional gene for L-gulono-gamma-lactone oxidase, the enzyme for L-ascorbic acid biosynthesis missing in man. Journal of Biological Chemistry, 269:13685-8.

Nusgens BV, Humbert P, Rougier A, Colige AC, Haftek M, Lambert CA, et al. (2001). Topically applied vitamin C enhances the mRNA level of collagens I and III, their processing enzymes and tissue inhibitor of matrix metalloproteinase 1 in the human dermis. Journal of Investigative Dermatology, 116:853-9.

Oberhammer F, Fritsch G, Schmied M, Pavelka M, Printz D, Purchio T, Lassmann H and Schulte-Hermann R. (1993). Condensation of the chromatin at the membrane of an apoptotic nucleus is not associated with activation of an endonuclease. Journal of Cell Science, 104:317-326.

Ohnishi M and Fujino Y. (1982). Sphingolipids in immature and mature soybeans. Lipids, 17(11):803-810.

Okazaki T, Bielawska A, Bell RM and.Hannun YA. (1990). Role of ceramide as a lipid mediator of 1-alpha,25dihydroxyvitamin D3-induced HL-60 cell differentiation. Journal of Bioligical Chemistry, 265:1582315831.

Okoshi H, Hakomori S, Nisar M, Zhou Q, Kimura S, Tashiro K and Igarashi Y. (1991) Cell membrane signalling as a target in cancer therapy II: Inhibitory effect of A/,AT,N-trimethylsphingosine on metastatic potential of murine B16 melanoma cell line through blocking of tumor cell-dependent platelet aggre gation. Cancer Research, 51: 6019-6024. 
Oren M, Maltzman W, Levine AJ. (1981). Post-translational regulation of the 54K cellular tumor antigen in normal and transformed cells. Molecular and Cellular Biology, 1:101-110.

Ouhtit A, Nakazawa H, Armstrong BK, Kricker A, Tan E, Hiroshi Yamasaki \& English, DR. (In press). UV-specific p53 mutation frequency in normal skin as potential predictor of non-melanocytic skin cancer risk: casecontrol study in australia. Journal of the National Cancer Institute.

P\&G Beauty \& Grooming. (2010). The sun. Retrieved May 14, 2011 from: http://www.pgbeautygroomingscience.c om/the-sun.html

Packer L, Weber SU, Rimbach G. (2001). Molecular aspects of alphatocotrienol antioxidant action and cell signaling. Journal of Nutrition, 131(Suppl):369-73S.

Parrish JA. Responses of skin to visible and ultraviolet radiation. In: Goldsmith LA, editor. Biochemistry and physiology of the skin. New York: Oxford University Press; 1983. p. 713-33.

Parsons PG, Hayward IP. (1985). Inhibition of DNA repair synthesis by sunlight. Photochemistry and Photobiology, 42:287-93.

Pasonen-Seppanen, S., Karvinen, S., Torronen, K., Hyttinen, J.M.T., Jokela, T., Lammi, M.J., Tammi, M.I. and Tammi, R. (2003). EGF upregulates, whereas TGF- $\beta$ downregulates, the hyaluronan synthases Has 2 and Has3 in organotypic keratinocyte cultures: correlations with epidermal proliferation and differentiation. Journal of Investigative Dermatology, 120: 1038-1044.

Pasquali M, Giraud F, et al. (2010). Toxin Induction and Protein Extraction from Fusarium spp. Cultures for Proteomic Studies. Journal of Visualized Experiments, (36): e1690.

Pathak MA, Riley FC and Fitzpatrick TB. (1962). Melanogenesis in human skin following exposure to long-wave ultraviolet and visible light. Journal. Investigative. Dermatology, 39:435-443.

Perez-Sala D, Collado-Escobar D, Mollinedo F. (1995). Intracellular alkalinization suppresses lovastatin-induced apoptosis in HL-60 cells through the activation of a $\mathrm{pH}$-dependent endonuclease. Journal of Biological Chemistry, 270:6235-6242

Pinnell SR, Yang HS, Omar M, Riviere NM, DeBuys HV, Walker LC, et al. (2001). Topical L-ascorbic acid: percutaneous absorption studies. Dermatological Surgery, 27:137-42.

Pinnell SR. (2003). Cutaneous photodamage, oxidative stress, and topical antioxidant protection. Journal of the American Academy of Dermatology, 48(1): 1-19

Podust VN, Podust LM, Goubin F, Ducommun B and Hubscher U. (1995). Mechanism of inhibition of proliferating cell nuclear antigen-dependent DNA synthesis by the cyclin-dependent kinase inhibitor p21. Biochemistry, 34:8869-8875

Polyak K, Lee MH, Erdjument--Bromage H, Koff A, Roberts JM, Tempst P and Massague J. (1994). Cloning of p27Kip1, a cyclin-dependent kinase inhibitor and a potential mediator of extracellular antimitogenic signals. Cell, 78(1):59-66.

Ponec M, Weerheim A, Kempenaar J, Mulder A, Gooris GS, Bouwstra J, Mommaas AM. (1997). The formation of competent barrier lipids in reconstructed human epidermis requires the presence of vitamin C. Journal of Investigative Dermatoligy, 109:348-355.

Potter JD. (1997). Cancer prevention: epidemiology and experiment. Cancer Letters, 114, 7-9.

Pourzand C, Tyrrell RM. (1999). Apoptosis, the role of oxidative stress and the example of solar UV radiation. Photochem Photobiol, 70, 380-390

QIAGEN Inc., AllPrep® DNA/RNA/Protein Mini Handbook, QIAGEN, Valencia, Calif, USA, 2007

Qiu Y, Wang W, Wang T, Liu J, Sun P, Qian J, Jin L and Xia Z. (2008). Genetic polymorphisms, messenger RNA expression of p53, p21, and CCND1, and possible links with chromosomal aberrations in chinese vinyl chloride-exposed workers. Cancer Epidemiology, Biomarkers \& Prevention, 17(10):2578-84. 
Rahn RO, Shulman RG and Longworth JW. (1965). The UV-induced triplet state in DNA. Proceedings of the National Academy of Sciences of the United States of America, 53, 893-896.

Rajadhyaksha M, Menaker G, et al. (2001). Confocal examination of nonmelanoma cancers in thick skin excisions to potentially guide mohs micrographic surgery without frozen histopathology. Journal of Investigative Dermatology, 117(5): 1137-1143.

Rajadhyaksha, M., S. Gonzalez, et al. (1999). In vivo confocal scanning laser microscopy of human skin II: advances in instrumentation and comparison with histology. Journal of Investigative Dermatology, 113(3): 293-303

Rasmussen RE and Painter RB. (1964). Evidence for repair of ultraviolet-damaged deoxyribonucleic acid in cultured mammalian cells. Nature, 203, 1360-1362.

Rebel H, Mosnier LO, et al. (2001). "Early p53-positive Foci as Indicators of Tumor Risk in Ultraviolet-exposed Hairless Mice: Kinetics of Induction, Effects of DNA Repair Deficiency, and p53 Heterozygosity." Cancer Research, 61(3): 977-983.

Rheinwald JG, Green H. (1977). Epidermal growth factor and multiplication of cultured human keratinocytes. Nature, 265:421-424.

Riley RT, Voss KA, Yoo H-S, Gelderblom WCA and Merrill AH. (1994b) Mechanism of fumonisin toxicity and carcinogenicity. Journal of. Food Protection. 57: 638-645

Rinaldi RA. (1960). The induction of pinocytosis in Amoeba proteus by ultraviolet radiation. Experimental Cell Research, 18. 70-75.

Robertson B, Gahring L, Newton R and Daynes RA. (1987). In vivo administration of IL-1 to normal mice decreases their capacity to elicit contact hypersensitivity responses: prostaglandins are involved in this modification of the immune response. Journal of.Investigative.Dermatology, 88, 380-387.

Rogel A, Popliker M, Webb CG, Oren M: p53 cellular tumor antigen: analysis of mRNA levels in normal adult tissues, embryos, and tumors. Molecular Cell Biology, 5:2851 $\pm 2855,1995$

Roshchupkin DI, Pistsov MYu, Potapenko AYa. Inhibition of ultraviolet light-induced erythema by antioxidants. Archives of Dermatological Research, 1979;266:91-4.

Rumsey SC, Wang Y, Levine M. Vitamin C. In: Papas AM, editor. Antioxidant status, diet, nutrition, and health. Boca Raton: CRC Press; 1999. p. 159-88.

Runger TM. (1999). Role of UVA in the pathogenesis of melanoma and non-melanoma skin cancer - a short review. Photodermatol Photoimmunol Photomed, 6: 212-6.

Rupert CS. (1964). Photoreactivation of ultraviolet damage. In Photophysiology, A. C. Giese (ed.). Academic Press Inc., New York, Vol. II, pp. 283-327.

Russell A, Laubscher A, et al. Investigating the protective properties of milk phospholipids against ultraviolet light exposure in a skin equivalent model. Multiphoton Microscopy in the Biomedical Sciences, X, SPIE.

Russo T, Zambrano N, et al. (1995). A p53-independent Pathway for Activation of WAF1/CIP1 Expression Following Oxidative Stress. Journal of Biological Chemistry, 270(49): 29386-29391.

Rustad RC. (1960). The centriole hypothesis of radiation-induced mitotic delay. Pathologie-Biologie, 9: 493-494.

Sackman M. (2000). Fluorescence Diagnosis in GI Endoscopy. Endoscopy, 32 (12): 977-985.

Sadahira Y, Ruan F, et al. (1992). Sphingosine 1-phosphate, a specific endogenous signaling molecule controlling cell motility and tumor cell invasiveness. Proceedings of the National Academy of Sciences of the United States of America, 89(20): 9686-9690

Sakakura C, Sweeney EA, et al. (1996). Suppression of Bcl-2 gene expression by sphingosine in the apoptosis of human leukemic HL-60 cells during phorbol ester-induced terminal differentiation. FEBS Letters, 379(2): 177-180. 
Sams WM, Smith JG and Burk PG. (1964). The experimental publication of elastosis with ultraviolet light. Journal of Investigative Dermatology, 43, 467-471.

Satyamoorthy K, Chehab NH, Waterman MJF, Lien MC, El-Deiry WS, Herlyn M, and Halazonetis TD. (2000). Aberrant Regulation and Function of Wild-Type p53 in Radioresistant Melanoma Cells. 11: 467-74.

Savini I, Catani MV, Rossi A, Duranti G, Melino G and Avigliano L. (2002). Characterization of keratinocyte differentiation induced by ascorbic acid: protein kinase $\mathrm{C}$ involvement and vitamin C homeostasis. Journal of Investigative Dermatology, 118: 372-379.

Schmelz EM. (2004). Sphingolipids in the chemoprevention of colon cancer. Frontiers in Bioscience, 9: 2632-2639

Schmelz EM, Crall KJ, et al. (1994). Uptake and metabolism of sphingolipids in isolated intestinal loops of mice. Journal of Nutrition, 124(5): 702-712.

Schomaker KT, Frisoli JK, Compton CC, Flotte TJ, Richter JM, Nishioka NS, et al. (1992). Ultraviolet laserinduced fluorescence of colonic tissue: basic biology and diagnostic potential. Lasers in Surgery and Medicine, 12: 63-78.

Seidl E and Clark W. (1978). The influence of ultraviolet radiation on the healthy adult. pp. 447-458. In: F. Urbach (ed.). Biologic Effects of Ultraviolet Radiation. Exeter: A. Wheaton \& Co.

Setlow RB and Setlow JK. (1962). Evidence that ultraviolet-induced thymine dimers in DNA cause biological damage. Proceedings of the National Academy of Sciences of the United States of America, 48: 1250-1257.

Shedden K and Cooper S. (2002) Analysis of cell-cycle-specific gene expression in human cells as determined by microarrays and double-thymidine block synchronization. Proceedings of the National Academy of Sciences of the United States of America, 99: 4379-4384.

Shindo Y, Hashimoto T. (1997). Time course of changes in antioxidant enzymes in human skin fibroblasts after UVA irradiation. Journal of Dermatological Science, 14: 225-32.

Shindo Y, Witt E, Packer L. (1993). Antioxidant defense mechanisms in murine epidermis and dermis and their responses to ultraviolet light. Journal of Investigative Dermatology, 100:260-5.

Silins I, Nordstrand M, Hogberg J and Stenius U. (2003). Sphingolipids suppress preneoplastic rat hepatocytes in vitro and in vivo. Carcinogenesis, 24, 1077-83.

Simon JD. (2000). Spectroscopic and Dynamic Studies of the Epidermal Chromophores trans-Urocanic Acid and Eumelanin. Accounts of Chemical Research, 33(5): 307-313

Singh RP and Agarwal R. (2005). Mechanisms and preclinical efficacy of silibinin in preventing skin cancer. European Journal of Cancer, 41(13): 1969-1979

Skin penetration depth of ultraviolet radiation of different wavelengths [Online Illustration]. (2010). Retrieved May 15, 2011 from NASA.gov. http://www.pgbeautygroomingsc ience.com/the-sun.html

Smith ML, Ford JM, et al. (2000). p53-Mediated DNA Repair Responses to UV Radiation: Studies of Mouse Cells Lacking p53, p21, and/or gadd45 Genes. Molecular and Cellular Biology, 20(10): 3705-3714

Soehnge H, Ouhtit A, et al. (1997). Mechanisms of induction of skin cancer by UV radiation. Frontiers in Bioscience, 2: 538-551

Sparmann G, Jaschke A, Loehr M, Liebe S, Emmrich J. (1997). Tissue homogenization as a key step in extracting RNA from human and rat pancreatic tissue. Biotechniques, 22: 408-10412.

Speers AE, Wu CC. (2007). Proteomics of integral membrane proteins--theory and application. Chem Rev. Aug;107(8): 3687-714. Review.

Spiegel S and Merrill AH. (1996). Sphingolipid metabolism and cell growth regulation. FASEB Journal 10(12): 1388-1397

Spiegel S and Milstien S. (2003). Sphingosine-1-phosphate: an enigmatic signaling lipid. Nature Reviews Molecular Cell Biology, 4: 397-4407. 
Sporn MB, Suh N. (2002). Chemoprevention: an essential approach in controlling cancer. Nature Reviews Cancer, 2: 537-543.

Stevens VL, Nimkar S, Jamison WC, Liotta DC and Merrill AH. (1990a). Characteristics of the growth inhibition and cytotoxicity of long-chain (sphingoid) bases for Chinese hamster ovary cells : evidence for an involvement of protein kinase C. Biochim Biophys Acta, 1051(1): 37-45.

Stevens VL, Owens NE, Winton EF, Kinkade JM and Merrill AH. (1990b). Modulation of retinoic acid-induced differentiation of human leukemia (HL-60) cells by serum factors and sphinganine. Cancer Res, 50(2): 222-6.

Streilein JW, Taylor JR, Vincek V, Kurimoto I, Shimizu T, Tie C, et al. (1994). Immune surveillance and sunlightinduced skin cancer. Immunol Today, 15: 174-9.

Strickland PT. (1986). Photocarcinogenesis by near-ultraviolet (UVA) radiation in Sencar mice. J Invest Dermatol, 87: 272-275.

Sugiki T, Tohnai N, Wang Y, Wada T and Inaki Y. (1996). Photodimerizations and crystal structures of thymine derivatives having a long alkyl chain connected with a carbamate bond. Bulletin of the Chemical Society of Japan, 69: 1777-1786. Retrieved from: http://www.chemistry.or.jp/journals/bcsj/JSTAGE/6906/pdf/69_1777.pdf

Swenson PA and Nishimura S. (1964). Inactivation of S-RNA by ultraviolet radiations. Photochemistry and Photobioliogy, 3: 85-90.

T. A. C. Society. (2009). Cancer Facts and Figures 2009. Retrieved May 22, 2011 from: http://www.cancer.org/acs/ groups/content/@nho/documents/document/500809webpdf.pdf

Tajima S and Pinnell SR. (1996). Ascorbic acid preferentially enhances type I and III collagen gene transcription in human skin fibroblasts. Journal of Dermatological Science, 11(3): 250-253.

Tan SC, Yiap BC. (2009). DNA, RNA, and protein extraction: the past and the present. Journal of Biomedicine and Biotechnology, 2009:1-10

Thiele JJ. Oxidative targets in the stratum corneum: a new basis for antioxidative strategies. Skin Pharmacology and Applied Skin Physiology, 2001;14:87-91.

Tommasi S, Swiderski PM, Tu Y, Kaplan BE \& Pfeifer GP. (1996). Inhibition of transcription factor binding by ultraviolet-induced pyrimidine dimers. Biochemistry, 35, 15693-703.

Tron VA, Trotter MJ, et al. (1998). p53-Regulated Apoptosis Is Differentiation Dependent in Ultraviolet BIrradiated Mouse Keratinocytes. American Journal of Pathology, 153(2): 579-585

Trosko JE, Chu EHY and Carrier WL. (1965). The induction of thymine dimers in ultraviolet-irradiated mammalian cells. Radiation Research, 24(4):667-672.

Tyrrell RM. (1991). "UVA (320-380 nm) radiation as an oxidative stress. In: Sies H, Oxidative stress, oxidants and antioxidants". London: Academic Press Ltd, p. 57-83.

Uchida Y, Behne M, Quiec D, Elias PM and Holleran WM. (2001). Vitamin C stimulates sphingolipid production and markers of barrier formation in submerged human keratinocyte cultures. Journal of Investigativev Dermatology, 117:1307-1313.

Ullrich SE. (1995). The role of epidermal cytokines in the generation of cutaneous immune reactions and ultraviolet radiation-induced immune suppression. Photochemistry and Photobiology, 62(3): 389-401.

Unden AB, Zaphiropoulos PG, Bruce K, Toftgard R \& Stahle-Backdahl M. (1997). Human patched (Ptch) mRNA is overexpressed consistently in tumor cells of both familial and sporadic basal cell carcinoma. Cancer Research, 57, 2336-40.

Urbach F. (1969). "The Biologic Effects of Ultraviolet Radiation." Exeter: A. Wheaton \& Co. 
U.S. Food and Drug Administration. (2011, June 14). FDA Announces Changes to Better Inform Consumers About Sunscreen. Retrieved June 23, 2011 from http://www.fda.gov/NewsEvents/Newsroom/PressAnnounc ements/ucm258940.htm

Van der Leun JC, and van Weelden H. "UVB Phototherapy: Principles, Radiation Sources, Regimens," Current. Problems in Dermatology, 15:39-51 (1986).

Vega MC, Saez IG, Aymami J, Eritja R, van der Marel GA, van Boom JH, Rich A and Coll M. (1994). Threedimensional crystal structure of the A-tract DNA dodecamer d(CGCAAATTTGCG) complexed with the minor-groove-binding drug Hoechst 33258. Eur. J. Biochem. 222, 721-726.

Vesper H, Schmelz E-M, et al. (1999). "Sphingolipids in Food and the Emerging Importance of Sphingolipids to Nutrition." Journal of Nutrition, 129(7): 1239-1250

Vidal MJ, Loganzo F, de Oliviera AR, Hayward NK and Albino NP. (1995). Mutations and defective expression of the WAF1 p21 tumour-suppressor gene in malignant melanomas. Melanoma Research, 5(4):243-250.

Vink AA, Moodycliffe AM, et al. (1997). The inhibition of antigen-presenting activity of dendritic cells resulting from UV irradiation of murine skin is restored by in vitro photorepair of cyclobutane pyrimidineâ€\%odimers. Proceedings of the National Academy of Sciences of the United States of America, 94(10): 5255-5260.

Vreeswijk MPG, van Hoffen A, Westland BE, Vrieling H, van Zeeland AA and Mullenders LHF. (1994). Analysis of repair of cyclobutane pyrimidine dimers and pyrimidine 6-4 pyrimidone photoproducts in transcriptionally active and inactive genes in Chinese hamster cells. Journal of. Biological Chemistry, 269: 31858-31863.

Vural P, Canbaz M, Selcuki D. (1999). Plasma antioxidant defense in actinic keratoses and basal cell carcinoma. Journal of the European Academy of Dermatology and Venereology, 113:96-101.

Wacker A, Dellweg H and Jacherts D. (1962). Thymin-Dimerisierung und Überlebenstrate bei Bakterien. Journal of Molecular Bioliogy, 4: 410-412.

Waga S, Hannon GJ, Beach D and Stillman B. (1994). The p21 inhibitor of cyclindependent kinases controls DNA replication by interaction with PCNA. Nature, 369: 574-578

Wang E, Norred WP, Bacon CW, Riley RT and Merrill AH. (1991) Inhibition of sphingolipid biosynthesis by fumonisins: implications for diseases associated with Fusarium moniliforme. Journal of Biological Chemistry, 266: 14486-14490.

Wang XU, Yeh H, Schaeffer L, Roy R, Moncollin V \& Egly JM. (1995). p53 modulation of TFIIH-associated nucleotide excision repair activity. Nature Genetics, 10: 188-95.

Wang ZY, M-T Huang, et al. (1994). Inhibitory Effects of Black Tea, Green Tea, Decaffeinated Black Tea, and Decaffeinated Green Tea on Ultraviolet B Light-induced Skin Carcinogenesis in 7,12Dimethylbenz[a]anthracene-initiated SKH-1 Mice. Cancer Research, 54(13): 3428-3435

Wargovich MJ, Lynch PM and Levin B. (1991). Modulating effects of calcium in animal models of colon carcinogenesis and short-term studies in subjects at increased risk for colon cancer. Amercian Journal of Clinical Nutrition, 54: 202S-205S.

Weissmann G and Dingle J. (1961). Release of lysosomal protease by UV irradiation and inhibition by hydrocortisone. Experimetnal Cell Research, 25: 207-210.

Wenczl E, Pool S, Timmerman AJ, Vanderschans GP, Roza L, Schothorst AA. (1997). Physiological doses of ultraviolet irradiation induce DNA strand breaks in cultured human melanocytes, as detected by means of an immunochemical assay. Photochemstry and Photobiology, 66: 826-30.

Weinstein IB. (1988). The origins of human cancer: molecular mechanisms of carcinogenesis and their implications for cancer prevention and treatment - twenty-seventh G.H.A. Clowes Memorial Award Lecture. Cancer Research, 48: 4135-4143. 
Wilkes GL, Brown IA and Wildnauer RH. (1973). The biomechanical properties of skin. CRC Critical Reviews in Bioengineering, 453-495.

Williams AC. (2003). Structure and function of human skin. In: Transdermal and Topical Drug Delivery - From theory to clinical practice. Pharmaceutical Press. Chicago.

Williams GT. (1991). Programmed cell death: Apoptosis and oncogenesis. Cell, 65, 1097-1098.

Wood RD. (1997). Nucleotide excision repair in mammalian cells. Journal of Biological Chemistry, 272, 2346523468.

Wu J, Spiegel S and Sturgill TW. (1995). Sphingosine-1-phosphate rapidly activates the MAP kinase pathway by a G-protein dependent mechanism. Journal of. Biological Chemistry. 270: 11484-11488.

Wulf HC, Stender IM, Lock-Andersen J. (1997). Sunscreens used at the beach do not protect against erythema: anewdefinition of SPF is proposed. Photodermatol Photoimmunol Photomed, 13:129-32.

Wurtman JJ. (1979). Sources of choline and lecithin in the diet. In Nutrition and the Brain-Choline and Lecithin in Brain Disorders (A. Barbeau, J. H. Growdon, and R. J. Wurtman, Eds.), Vol. 5, pp. 73-81. Raven Press, New York.

Wyllie AH, Kerr JFR and Currie AR. (1980). Cell death: The significance of apoptosis. International Review of Cytology, 68: 251-306.

Xia J, Song X, Bi Z, Chu W and Wan Y. (2005). UV-induced NF-kappaB activation and expression of IL-6 is attenuated by (-)-epigallocatechin-3-gallate in cultured human keratinocytes in vitro. Internatiolnal Journal of Molecular Medicine, 16(5):943-50.

Xu CX, Green A, Parisi A, Parsons PG. (2001). Photosensitization of the sunscreen octyl p-dimethylaminobenzoate by UVA in human melanocytes but not in keratinocytes. Photochemistry and Photobiology, 73:600-4.

Yoshikawa T and Streilein JW. (1991) Tumor necrosis factor-alpha and ultraviolet B light have similar effects on contact hypersensitivity in mice. Regional Immunology, 3, 139-144.

Young IS, Woodside JV. (2001). Antioxidants in health and disease. Journal of Clinical Pathology, 54:176-86.

Yuen KS, Halliday GM. (1997). Alpha-tocopherol, an inhibitor of epidermal lipid peroxidation, prevents ultraviolet radiation from suppressing the skin immune system. Photochemistry and Photobiology, 65:587-92.

Zackheim HS. (1963). Origin of the human basal cell epithelioma. Journal of Investigative. Dermatology. 40, 28397.

Zambetti GP and Levine AJ. (1993). A comparison of the biological activities of wild-type and mutant p53. The FASEB Journal, 7(10): 855-865.

Zeisel SH, Char D and Shear NF. (1986). Choline, phosphatidylcholine and sphingomyelin in human and bovine milk and infant formulas. Journal of Nutr, 116: 50-58.

Zeng Y-X and El-Deiry WS. (1996). Regulation of p21 $1^{\mathrm{WAF} 1 / \mathrm{CIP} 1}$ expression by p53- independent pathways. Oncogene, 12: 1557-1564

Zenisck A, Kral JA and Hais IM. (1955). 'Sun-screening' effect of urocanic acid. Biochimica et. Biophysica. Acta, $18,589-591$.

Zhang H, Buckley NE, et al. (1990). Sphingosine stimulates cellular proliferation via a protein kinase C-independent pathway. Journal of Biological Chemistry, 265(1): 76-81.

Zhang H, Desai NN, et al. (1991). Sphingosine-1-phosphate, a novel lipid, involved in cellular proliferation. The Journal of Cell Biology, 114(1): 155-167

Zheng Q, Song J, Doncaster K, Rowland E and Byers DM. (2007). Qualitative and quantitative evaluation of protein extraction protocols for apple and strawberry fruit suitable for two-dimensional electrophoresis and mass spectrometry analysis. Journal of Agricultural and Food Chemistry, 55: 1663-73.

Ziegler A, Jonason AS, et al. (1994). Sunburn and p53 in the onset of skin cancer. Nature, 372(6508): 773-776. 
Ziegler A, Leffell DJ, et al. (1993). Mutation Hotspots Due to Sunlight in the p53 Gene of Nonmelanoma Skin Cancers. Proceedings of the National Academy of Sciences of the United States of America, 90(9): 42164220

Zigman S, Fowler $\mathrm{J}$ and Kraus $\mathrm{AL}$. Black light induction of skin tumors in mice. Journal of Investigative Dermatology, 67, 723-25 (1976)

Zong W-X, Lindsten T, et al. (2001). BH3-only proteins that bind pro-survival Bcl-2 family members fail to induce apoptosis in the absence of Bax and Bak. Genes \& Development, 15(12): 1481-1486

Zuang V, Alonso M-A, Botham PA, et al. (2005). Skin irritation and corrosion. Alternative to Laboratory Animals, 33, Suppl. 1, 35-46. 


\section{Glossary}

Adenoma - benign tumor that develops from epithelial tissue

Adenocarcinoma - malignant tumor of a gland

Agonist - substance that initiates a physiological response when combined with a receptor

Alleles - one of two or more alternative forms of a gene located on the same place on a chromosome

Anabolism - synthesis of complex molecules from simple ones with accompanying storage of energy

Antigen - any substance capable of inducing a specific immune response and of reacting with the products of that response

Benign - of no danger to health

Bleed-through - emission detected in the channel or through the filter combination reserved for a second

fluorophore

Cell line - culture of cells with an indefinite lifespan and which are considered immortal

Carcinoma - malignant growth of epithelial cell tissues, capable of infiltrating surrounding tissues and giving rise to metastases

Catabolism - breakdown of complex molecules to form simpler ones accompanied by a release of energy

Cecum - cavity in which the large intestine vegins

Chromatin - mass of genetic material composed of DNA and proteins that condense to form chromosomes during eukaryotic cell division

Complete carcinogen - an agent capable of inducing cancer without the need for a mediator that promotes tumorigenesis

Cutaneous - of, relating to, or affecting the skin

Cytokine - any number of substances secreted by certain cells of the immune system and having an effect on other cells

Desquamation - shedding of epithelial elements, chiefly of the skin, in scales or sheets

Differentiation - process by which cells become progressively more specialized

Deletion - Removal of a chromosomal segment from a chromosome set.

Duplication - More than one copy of a particular chromosomal segment in a chromosome set. 
Dyskeratosis - abnormal, premature, or imperfect keratinization of the keratinocytes

Epigenetic - resulting from external rather than genetic influences

Erythrocyte - a cell that contains hemoglobin and can carry oxygen to the body. Also called a red blood cell (RBC)

Eukaryotic - having cell with membrane-bound nuclei

Exon - a segment of DNA or RNA molecule containing information coding for a protein

Expression - translation of information encoded in a gene into protein or RNA

Frameshift mutation - shift in the way the DNA sequence is read due to a deletion or insertion of a nucleotide

Free radicals - atoms or molecules with an unpaired electron

Genome - the complete set of genetic material of an organism

HaCaT cells - are human skin keratinocytes that have been transformed to become immortal. These cells have mutated both p53 alleles

Hemolysis - the destruction of red blood cells which leads to the release of hemoglobin from within the red blood cells into the blood plasma

Histone - nuclear proteins which function to pack and order the DNA into structural units known as nucleosomes

Homeostasis - the process whereby the internal environment of an organism tends to remain balanced and stable

Hydrophilic - having affinity for water; readily absorbing or dissolving in water

Hydrophobic - tending to repel or faili to mix with water

Hypospadia - congenital defect in male urethra that involves opening of the urethra under the penis shaft

Hypoxia - deficiency in the amount of oxygen reaching the tissues

Lesion - A damaged area in a gene (a mutant site), a chromosome, or a protein.

Li-Fraumeni Syndrome - group of symptoms generally exhibiting a great diversity of tumors

Ligand - substance able to bind to and form a complex with a biomolecule to serve a biological purpose

Lymphocyte - a mononuclear, non-granular type of white blood cell normally found in the blood and the lymph systems

Lysosome - membrane-bound organelle in the cytoplasm of most cells containing various hydrolytic enzymes that function in intracellular digestion

Kinase - enzyme that catalyzes the transfer of a phosphate froup from ATP to a specified molecule 
Macrophages - large phagocytic cell found in stationary form in the tissues or as a mobile white blood cell, esp. at sites of infection

Malignant - tending to invade normal tissue or to recur after removal; cancerous

Melanization - to convert into melanin or to increase the concentration of melanin within

Monomer - simplest or the repeating unit of a polymer

Murine - of, relating to, or affecting mice or related rodents

Mitogenic - any substance that triggers mitosis, as well as lymphocyte blastogenesis

Organelle - any number of organized or specialized structures within a living cell

Papillomas - benign epithelial tumor growth with specific morphology

PCNA - proliferating cell nuclear antigen; protein that acts as a processivity factor for DNA polymerase $\delta$ in eukaryotic cells

Phenotype - set of observable characteristics of an individual resulting from interaction of fits genotype with the environment

Pinocytosis - introduction of fluids into a cell by invagination of the cell membrane, followed by formation of vesicles within the cells

Promoter Sequence -regulatory DNA sequence that initiates the expression of a gene

Purine - bases adenine and guanine present in DNA and RNA

Pyrimidine - A type of nitrogen base; the pyrimidine bases in DNA are cytosine and thymine.

Solublize - make a substance more soluble

Syngeneic - genetically similar or identical and hence, immunologically compatible

Transcription - process of creating an equivalent RNA copy of a sequenc of DNA

Transformation (oncogenic) - indefinite growth of previously normal cells which can be caused by spontaneous mutations leading to oncogenesis

Transversion - A type of nucleotide-pair substitution in which a pyrimidine replaces a purine or vice versa—for example, G.C $\rightarrow$ T.A. 


\section{Appendix}

\section{Reagents and Media Components}

$1 \%$ BSA $(50 \mathrm{~mL})$, store at $4^{\circ} \mathrm{C}$

$0.5 \mathrm{~g}(1 \% \mathrm{w} / \mathrm{v})$ Bovine Serum Albumin (BSA)

Dilute to $50 \mathrm{~mL}$ with $\mathrm{ddH}_{2} \mathrm{O}$

Urea Lysis Buffer $(\mathbf{5 0} \mathrm{mL})$, store at $\mathbf{- 2 0 ^ { \circ } \mathrm { C }}$

$0.5 \mathrm{~g}(1 \% \mathrm{w} / \mathrm{v})$ sodium dodecyl sulfate (SDS)

$27.027 \mathrm{~g}(9 \mathrm{M})$ urea

$0.197 \mathrm{~g}(25 \mathrm{mM})$ Tris- $\mathrm{HCl}, \mathrm{pH}=6.8$

$0.0186 \mathrm{~g}(1 \mathrm{mM})$ ethylenediaminetetraacetic acid (EDTA)

$2.735 \mathrm{~g}(0.7 \mathrm{M})$ 2-mercaptoethanol

Dilute to $50 \mathrm{~mL}$ with $\mathrm{ddH}_{2} \mathrm{O}$

$1 \%$ NP-40 Lysis Buffer ( $50 \mathrm{~mL}$ ), store at room temperature

$0.5 \mathrm{~mL}(1 \% \mathrm{v} / \mathrm{v}) \mathrm{NP}-40$ or Triton-X 100 detergent

$0.438 \mathrm{~g}(150 \mathrm{mM}) \mathrm{NaCl}$

$0.394 \mathrm{~g}(50 \mathrm{mM})$ Tris- $\mathrm{HCl}, \mathrm{pH}=8.0$

$0.25 \mathrm{~g}(0.5 \% \mathrm{w} / \mathrm{v})$ sodium deoxycholate

$0.05 \mathrm{~g}(0.1 \% \mathrm{w} / \mathrm{v}) \mathrm{SDS}$

1 tablet Complete mini protease inhibitor cocktail* (Roche, Indianapolis, IN)

Dilute to $50 \mathrm{~mL}$ with ddH2O

* add components immediately prior to use

2\% SDS Lysis Buffer $(\mathbf{5 0} \mathbf{~ m L})$, store at $-20^{\circ} \mathrm{C}$

$0.394 \mathrm{~g}$ Tris- $\mathrm{HCl}(0.05 \mathrm{M}), \mathrm{pH}=8.0$

$1 \mathrm{~g}(2 \% \mathrm{w} / \mathrm{v})$ SDS

$0.077 \mathrm{~g}(10 \mathrm{mM})$ DTT (dithiothreitol)

$0.002 \mathrm{~g}(0.01 \mathrm{mM})$ EDTA (ethylenediaminetetraacetic acid) or $20 \mu \mathrm{L}$ from $500 \mathrm{mM}$ stock solution

$0.009 \mathrm{~g}(1 \mathrm{mM})$ PMSF (phenylmethylsulfonyl fluoride)* or $200 \mu \mathrm{L}$ from a $500 \mathrm{mM}$ stock solution

1 tablet Complete mini protease inhibitor cocktail* (Roche, Indianapolis, IN)

Dilute to $50 \mathrm{~mL}$ with $\mathrm{ddH}_{2} \mathrm{O}$

* add components immediately prior to use

TCA/acetone Precipitation Buffer $(15 \mathrm{~mL})$, store at $4^{\circ} \mathrm{C}$

$3 \mathrm{~g}(20 \% \mathrm{w} / \mathrm{v}) \mathrm{TCA}$ (trichloroacetic acid)

$0.015 \mathrm{~g}(0.1 \% \mathrm{w} / \mathrm{v}) \mathrm{DTT}$

Dilute to $15 \mathrm{~mL}$ with Acetone

Wash Buffer $(15 \mathrm{~mL})$, store at $4^{\circ} \mathrm{C}$

$0.015 \mathrm{~g}(0.1 \% \mathrm{w} / \mathrm{v}) \mathrm{DTT}$

Dilute to $15 \mathrm{~mL}$ with Acetone

TBS - Tris Buffered Saline $(100 \mathrm{~mL})$, store at Room Temperature (RT)

$0.6 \mathrm{~g}(0.05 \mathrm{M})$ Tris base or $0.788 \mathrm{~g}$ Tris- $\mathrm{HCl}$

$0.87 \mathrm{~g}(150 \mathrm{mM}) \mathrm{NaCl}$

$\mathrm{pH}=7.4$ (Adjust with $\mathrm{HCl}$ or $\mathrm{NaOH}$ )

Dilute to $100 \mathrm{~mL}$ with $\mathrm{ddH}_{2} \mathrm{O}$

TBS-Tween (1 L), store at RT

1 L TBS

$5 \mathrm{~mL}$ Tween-20 
Bradford Reagent $(50 \mathrm{~mL})$, store at $4^{\circ} \mathrm{C}$

$(0.01 \%$ w/v) Coomassie Brilliant Blue G-250

$(4.7 \% \mathrm{v} / \mathrm{v})$ ethanol

(8.5\% v/v) phosphoric acid

Dilute to $50 \mathrm{~mL}$ with $\mathrm{ddH}_{2} \mathrm{O}$

Laemmli 2X Buffer, freshly made

$4 \%$ SDS

$10 \%$ 2-mercaptoethanol

$20 \%$ glycerol

$0.004 \%$ bromophenol blue

$0.125 \mathrm{M}$ Tris- $\mathrm{HCl}$

Adjust to $\mathrm{pH} 6.8$

Dilute to desired volume with ddH2O such that ratio of buffer volume to protein volume is $1: 1$

Lower Buffer $(250 \mathrm{~mL})$, store at $4^{\circ} \mathrm{C}$

$45.43 \mathrm{~g}(1.5 \mathrm{M})$ Tris

$1 \mathrm{~g}(0.4 \% \mathrm{w} / \mathrm{v}) \mathrm{SDS}$

Adjust to $\mathrm{pH} 8.8$

Diliute to $250 \mathrm{~mL}$ with ddH2O

Upper Buffer $(250 \mathrm{~mL})$, store at 4oC

$15.14 \mathrm{~g}(0.5 \mathrm{M})$ Tris

$1 \mathrm{~g}(0.4 \% \mathrm{w} / \mathrm{v}) \mathrm{SDS}$

Adjust to $\mathrm{pH} 6.8$

Dilute to $250 \mathrm{~mL}$ with ddH2O

Running Buffer (25 mM Tris, $192 \mathrm{mM}$ glycine, 0.1\% w/v SDS, pH 8.3, $1 \mathrm{~L}$ ), store at RT

Dilute $100 \mathrm{~mL}$ Bio-Rad 10X Tris/glycine/SDS running buffer to $1 \mathrm{~L}$ with ddH2O

Solution can be reused up to 5 times

Coomassie Blue Stain $(200 \mathrm{~mL})$, store at $4^{\circ} \mathrm{C}$

$0.5 \mathrm{~g}(0.2 \% \mathrm{w} / \mathrm{v})$ Coommassie Brilliant Blue R-250

$100 \mathrm{~mL}(50 \% \mathrm{v} / \mathrm{v})$ methanol

$20 \mathrm{~mL}(10 \% \mathrm{v} / \mathrm{v})$ acetic acid

Dilute to $200 \mathrm{~mL}$ with $\mathrm{ddH}_{2} \mathrm{O}$

Solution can be saved and reused many times

Destaining Solution $(250 \mathrm{~mL})$, store at RT

$25 \mathrm{~mL}(10 \% \mathrm{v} / \mathrm{v})$ acetic acid

$125 \mathrm{~mL}(50 \% \mathrm{v} / \mathrm{v})$ methanol

Dilute to $250 \mathrm{~mL}$ with ddH2O

\section{Storage Solution $(\mathbf{2 6 0} \mathrm{mL})$, store at RT}

$25 \mathrm{~mL}(10 \% \mathrm{v} / \mathrm{v})$ acetic acid

Dilute to $250 \mathrm{~mL}$ with ddH2O

Buffer A (250 mL), store at RT

$9.085 \mathrm{~g}(0.3 \mathrm{M})$ Tris Base

Adjust to $\mathrm{pH} 10.4$

Dilute to $250 \mathrm{~mL}$ with ddH2O

Buffer B (250 mL), store at RT

$0.76 \mathrm{~g}(0.025 \mathrm{M})$ Tris Base 
Adjust to $\mathrm{pH} 10.4$

Dilute to $250 \mathrm{~mL}$ with ddH2O

\section{Buffer C (250 mL), store at RT}

$0.76 \mathrm{~g}(0.025 \mathrm{M})$ Tris Base

$1.312 \mathrm{~g}(0.4 \mathrm{M})$ 6-amino-n-hexanoic acid

Adjust to $\mathrm{pH} 9.4$

Dilute to $250 \mathrm{~mL}$ with ddH2O

\section{$2.5 \%$ gelatin in TBS-Tween $(100 \mathrm{~mL})$, freshly made}

$100 \mathrm{~mL}$ TBS-Tween

$2.5 \mathrm{~g}(3 \% \mathrm{w} / \mathrm{v})$ gelatin (Product No. 170-6537, Bio-Rad Laboratories, Hercules, CA)

Place in beaker and heat solution while stirring at $80 \mathrm{oC}$ for approximately 30 minutes then leave to cool on a stir plate for around 1 hour

\section{$1 \%$ BSA in TBS-Tween $(100 \mathrm{~mL})$, freshly made}

$100 \mathrm{~mL}$ TBS-Tween

$1 \mathrm{~g}$ bovine serum albumin (BSA)

\section{TBS Blotto A $(500 \mathrm{~mL})$, store at $40 \mathrm{C}$ for up to two days or store at $\mathbf{- 2 0 ^ { \circ } \mathrm { C }}$}

Dissolve the entire contents of the blocking buffer bottle (TBS Blotto A, Santa Cruz Biotechnology, Santa Cruz, $\mathrm{CA}$ ) in dd $\mathrm{H} 2 \mathrm{O}$ by adding powder slowly to water while stirring

Dilute to a final volume of $500 \mathrm{~mL}$

Add the entire contents of the Tween-20 vial with a pipette

Mix until completely dissolved

\section{5\% Casein $(100 \mathrm{~mL})$, freshly made}

$0.5 \mathrm{~g}(0.5 \% \mathrm{w} / \mathrm{v})$ casein (Hammarsten grade)

Dillute to $100 \mathrm{~mL}$ with TBS-Tween

Heat solution while stirring at $\sim 80 \mathrm{oC}$ for $\sim 30$ minutes then transfer to cool stir plate for $\sim 1$ hour

\section{Peroxidase-conjugated Goat Anti-Rabbit secondary antibody (20 (L), store at $-80^{\circ} \mathrm{C}$}

Reconstitute a vial of Peroxidase AffiniPure goat anit-rabbit IgG $(\mathrm{H}+\mathrm{L})$ in $2 \mathrm{~mL}$ ddH2O to get $1 \mathrm{mg} / \mathrm{mL}$ concentration

Aliquot solution in $20 \mu \mathrm{L}$ fractions and store indefinitely at $-80 \mathrm{oC}$

\section{Diaminobenzidine substrate $(20 \mathrm{~mL})$, freshly made}

Weigh 12 mg DAB powder (Sigma Aldrich, St. Louis, MO)

Diliute in $20 \mathrm{~mL}$ TBS

Add $25 \mu \mathrm{L} 30 \%$ hydrogen peroxide (H2O2) (Mallinckrodt Laboratory Chemicals, Charlotte, NC)

Stir until DAB dissolves

SuperSignal West Pico Substrate Kit $(1 \mathrm{~mL})$, freshly made stable for 24 hours at RT in the dark $0.5 \mathrm{~mL}$ of the Stable Peroxide Solution

$0.5 \mathrm{~mL}$ of the Luminol/Enhancer Solution

Use $0.1 \mathrm{~mL}$ working Solution per $\mathrm{cm}^{2}$ membrane 